\title{
Human Reproductive Health in Relation to Thyroid Alterations
}

\author{
Thushani Siriwardhane ${ }^{1 *}$, Karthik Krishna², Qi Song², Vinodh Ranganathan², \\ Vasanth Jayaraman², Tianhao Wang2, Kang Bei², John J. Rajasekaran², Hari Krishnamurthy ${ }^{2 *}$ \\ ${ }^{1}$ Vibrant America LLC, San Carlos, CA, USA \\ ${ }^{2}$ Vibrant Sciences LLC, San Carlos, CA, USA \\ Email: *thushanis@vibrant-america.com, *hari@vibrantsci.com
}

How to cite this paper: Siriwardhane, T., Krishna, K., Song, Q., Ranganathan, V., Jayaraman, V., Wang, T.H., Bei, K., Rajasekaran, J.J. and Krishnamurthy, H. (2019) Human Reproductive Health in Relation to Thyroid Alterations. Health, 11, 1095-1133. https://doi.org/10.4236/health.2019.118086

Received: July 16, 2019

Accepted: August 27, 2019

Published: August 30, 2019

Copyright $\odot 2019$ by author(s) and Scientific Research Publishing Inc. This work is licensed under the Creative Commons Attribution-NonCommercial International License (CC BY-NC 4.0). http://creativecommons.org/licenses/by-nc/4.0/

\begin{abstract}
Background: Thyroid alterations have adverse effects on other health systems in the body, and reproductive system is one of the most affected organs. Here, we perform a comprehensive analysis on the effects of different thyroid abnormalities on sexual and reproductive-related hormones in both men and women in different age groups. Method: A comprehensive analysis was performed on 15,043 subjects within the reproductive age (15 - 49 years). They were tested for thyroid markers (TSH, FT4, anti-TPO, anti-Tg) and 13 reproductive hormones in Vibrant America Clinical Laboratory between March 2016 to July 2018. The alterations of each reproductive hormone in hypothyroidism, hyperthyroidism, anti-TPO+ anti-Tg+ groups were evaluated. Results: A total of 10,626 women was tested. Women with hypothyroidism had elevated cortisol and low SHBG and DHEA-S. Women with hyperthyroidism showed elevated total testosterone and SHBG. Women seropositive for anti-TPO had elevated total testosterone and low cortisol. Women seropositive for anti-Tg had low parathyroid hormones. Similarly, a total of 4417 men was tested. Men with hypothyroidism had low IGF-I. Similarly, men with hyperthyroidism had low DHEA-S, but elevated estradiol, FSH, LH and prolactin. Men seropositive for anti-TPO had elevated SHBG and low progesterone. Men seropositive for anti- $\mathrm{Tg}$ had elevated progesterone. The reproductive and related hormone levels of age group 36 - 49 showed the most variations. Conclusion: Our results clearly show that individuals with different thyroid alterations have different effects on reproductive health, especially in the age group 36 - 49. Hence, routine testing and follow-up checkups on reproductive system would be beneficial for individuals in the age group 36 - 49 with thyroid disorders.
\end{abstract}

\section{Keywords}

Reproductive Hormones, Hypothyroidism, Hyperthyroidism, Thyroid Disease, 
Thyroid Autoantibodies

\section{Introduction}

Reproductive hormones are responsible for several aspects of human sexual behaviors. Imbalances in steroid hormones may lead to impaired sexual function and decreased fertility in both men and women [1] [2]. Particularly, women suffer from menstrual irregularities, miscarriages, adverse pregnancy and neonatal outcomes while men suffer from abnormal sperm morphology, changes in sperm motility, etc. Thyroid disease is considered one of the main endocrine conditions responsible for hormone imbalances in both men and women affecting their reproductive system. These endocrine systems have been linked since the functions of both thyroid gland and gonadal system are under the control of the hypothalamus-pituitary-gonadal axis.

Thyroid disease is mainly categorized as hypothyroidism and hyperthyroidism and both these conditions can have adverse effects on reproductive health. In women, hypothyroidism is responsible for subfertility in approximately $2.3 \%$ compared to the $1.5 \%$ incidence rate of women in the general population [3]. Hypothyroidism is commonly observed in pregnancy with a $0.5 \%$ overt disease and a $2.5 \%$ subclinical disease in pregnant women [3]. Graves' disease, which is the common form of hyperthyroidism in pregnancy has been observed in $1 \%$ of pregnant women [3]. In men, hypothyroidism causes abnormal sperm morphology, reduced sperm motility, impaired sexual behaviors, including hypoactive sexual desire (HSD), erectile dysfunction (ED) and ejaculatory disorders [4]. Hyperthyroid men were reported with significant reduction in semen volume, sperm count, sperm motility, number of morphologically normal sperm, gynecomastia as well as impaired sexual behaviors similar to hypothyroid men [4].

Anti-TPO and anti-Tg are commonly observed in autoimmune thyroid disease (AITD) has gained increased attention over the past years. Women with AITD have been associated with lower fertilization rates, poorer embryo quality, and lower pregnancy rates [5]. The presence of thyroid autoantibodies, in euthyroid subjects was reported to have a threefold increase in the odds ratio of miscarriages compared to healthy women [6]. Women with AITD have been suggested to have a $5 \%-10 \%$ risk of developing hypothyroidism in pregnancy with a 2 - 4-fold increased risk of preterm labor [3]. Unfortunately, the association of reproductive health in male subjects with thyroid antibodies has been poorly studied. Some studies reported that the presence of elevated levels of anti-TPO may be associated with pathozoospermia or asthenozoospermia [7].

It is crucial to study the effects of different thyroid dysfunctions on reproductive health in both genders. Numerous studies have shown the effect of thyroid disease on reproductive hormones, but each study only focused on one subcategory of thyroid disorder. None of the studies compared these common subcate- 
gories of thyroid disease in relation to sexual and reproductive-related hormones in both genders in one study. Since the upper limit of the TSH reference range is currently debatable, and typically based on the laboratory reference levels, a comprehensive study between these common subcategories of thyroid disorder would provide considerably meaningful comparative results.

In this study, we performed a comprehensive analysis including the common thyroid subcategories (subclinical/overt hypothyroidism and subclinical/overt hyperthyroidism) and autoantibodies related to AITD separately for 13 sexual and reproductive-related hormones for a large population size of 15,043 including 10,626 women and 4417 men in different reproductive age groups. Their serology was used to evaluate the association of sexual and reproductive-related hormones with thyroid disorders in both genders in different ages. Our results showed different alterations in sexual and reproductive related-hormone levels in different thyroid subcategories thus evidencing that different thyroid dysfunctions could complicate the regular functions of the reproductive system in different ways.

\section{Material and Methods}

\subsection{Patient Selection and Study Design}

A total of 10,626 women and 4417 men between age 15 - 49 were tested in the Vibrant America Clinical Laboratory for thyroid markers (TSH, FT4, anti-TPO, anti-Tg) between March 2016 to July 2018. Same cohort was simultaneously tested for 13 reproductive and other related hormones. Estradiol, FSH, LH and Progesterone were not included in the analysis for women since the reference ranges were depending on women hormonal phases. This retrospective analysis was completed using de-identified laboratory test results. Demographics of subjects in each group is listed in Table 1. Inclusion and exclusion criteria are listed below.

Inclusion Criteria:

- Subjects between age $15-49$.

- Subjects who tested both TSH and FT4 or anti-TPO or anti-Tg at each visit. Exclusion Criteria:

- Subjects age $<15$ years and $49<$ years.

- Subjects who did not test for both TSH and FT4 together or anti-TPO or anti-Tg at each visit.

The physician reported ICD-10-CM (International Classification of Diseases, Tenth Revision, Clinical Modification) codes were used to provide clinical information on these subjects. Subjects were divided into subgroups depending on the presence of thyroid hormones and autoantibody.

Hypothyroidism-Subjects with either subclinical or Overt hypothyroidism.

Hyperthyroidism-Subjects with either subclinical or Overt hyperthyroidism.

Thyroid negative (thyroid)_Subjects with normal levels of TSH and FT4 (control group for hypothyroidism and hyperthyroidism). 
Anti-TPO positive (anti-TPO+)-Subjects with increased levels of anti-TPO antibodies.

Anti-TPO negative (anti-TPO-)-Subjects with normal levels of anti-TPO antibodies (control group for anti-TPO+ group).

Anti-Tg positive (anti- $\mathrm{Tg}+$ ) - Subjects with increased levels of anti- $\mathrm{Tg}$ antibodies.

Anti-Tg positive (anti-Tg-) -Subjects with normal levels of anti-Tg antibodies (control group for anti-Tg+ group).

These subcategories were evaluated for sexual and reproductive-related hormone levels in different age groups (15 - 49, 15 - 25, 26 - 35, 36 - 49 years).

\subsection{TSH, FT4, Anti-TPO and Anti-Tg Tests}

TSH, FT4, anti-TPO and anti-Tg were measured using the commercial Roche e601 Analyzer, (Roche Diagnostics, Indianapolis, IN, USA) according to the manufacturer's recommendations. All reagents were purchased from Roche Diagnostics (Indianapolis, IN, USA). Human serum specimens were used on Elecsys immunoassay analyzers (Roche Diagnostics, Indianapolis, IN, USA).

Table 1. Demographics of subjects in each group.

\begin{tabular}{|c|c|c|c|c|c|c|c|}
\hline & Hypothyroidism & Hyperthyroidism & Thyroid- & Anti-TPO+ & Anti-TPO- & Anti-Tg+ & Anti-Tg \\
\hline \multicolumn{8}{|l|}{ Age $15-49$} \\
\hline \multicolumn{8}{|l|}{ Female } \\
\hline Age $(\mathrm{X} \pm \mathrm{SD})$ & $37 \pm 9$ & $39 \pm 8$ & $36 \pm 9$ & $37 \pm 9$ & $37 \pm 9$ & $37 \pm 9$ & $36 \pm 9$ \\
\hline \multicolumn{8}{|l|}{ Male } \\
\hline Age $(\mathrm{X} \pm \mathrm{SD})$ & $36 \pm 9$ & $40 \pm 8$ & $36 \pm 9$ & $37 \pm 9$ & $36 \pm 9$ & $37 \pm 9$ & $36 \pm 9$ \\
\hline \multicolumn{8}{|l|}{ Age $15-25$} \\
\hline \multicolumn{8}{|l|}{ Female } \\
\hline Age $(\mathrm{X} \pm \mathrm{SD})$ & $21 \pm 3$ & $21 \pm 3$ & $21 \pm 3$ & $21 \pm 3$ & $21 \pm 3$ & $21 \pm 3$ & $21 \pm 3$ \\
\hline \multicolumn{8}{|l|}{ Male } \\
\hline Age $(\mathrm{X} \pm \mathrm{SD})$ & $21 \pm 3$ & $21 \pm 2$ & $20 \pm 3$ & $21 \pm 3$ & $20 \pm 3$ & $21 \pm 3$ & $20 \pm 3$ \\
\hline \multicolumn{8}{|l|}{ Age $26-35$} \\
\hline \multicolumn{8}{|l|}{ Female } \\
\hline Age $(X \pm S D)$ & $31 \pm 3$ & $31 \pm 3$ & $31 \pm 3$ & $31 \pm 3$ & $31 \pm 3$ & $31 \pm 3$ & $31 \pm 3$ \\
\hline \multicolumn{8}{|l|}{ Male } \\
\hline Age $(X \pm S D)$ & $30 \pm 3$ & $32 \pm 3$ & $31 \pm 3$ & $31 \pm 3$ & $31 \pm 3$ & $31 \pm 3$ & $31 \pm 3$ \\
\hline \multicolumn{8}{|l|}{ Age $36-49$} \\
\hline \multicolumn{8}{|l|}{ Female } \\
\hline Age $(X \pm S D)$ & $43 \pm 4$ & $44 \pm 4$ & $43 \pm 4$ & $43 \pm 4$ & $43 \pm 4$ & $43 \pm 4$ & $43 \pm 4$ \\
\hline \multicolumn{8}{|l|}{ Male } \\
\hline Age $(\mathrm{X} \pm \mathrm{SD})$ & $43 \pm 4$ & $44 \pm 4$ & $43 \pm 4$ & $43 \pm 4$ & $43 \pm 4$ & $43 \pm 4$ & $43 \pm 4$ \\
\hline
\end{tabular}

$\mathrm{X}-$ Average; SD-Standard Deviation. 
Specific TSH monoclonal antibodies Specifically directed against human TSH were employed in the Elecsys TSH assay (Roche Diagnostics, Indianapolis, IN, USA). The antibodies labeled with a ruthenium complex consist of a chimeric construct from human and mouse-specific components. As a result, interfering effects due to HAMA (human anti-mouse antibodies) were largely eliminated.

The Elecsys FT4 test employed a specific anti-T4 antibody labeled with a ruthenium complex to determine the free thyroxine. The quantity of antibody used was so small (equivalent to approx. $1 \%-2 \%$ of the total T4 content of a normal serum sample) that the equilibrium between bound and unbound T4 remained virtually unaffected.

Elecsys anti-TPO assay employed recombinant antigens and polyclonal anti-TPO antibodies whereas Elecsys anti-Tg assay employed monoclonal human anti-Tg antibodies.

\subsection{Reference Ranges for Thyroid Markers}

Thyroid hormone reference ranges are subject to the lab where the test is performed. In this study, we used the reference ranges that majority of the commercial test labs and hospital labs use. The reference range of thyroid markers in a healthy control used in this study is shown in Table 2 .

The categorization of serologic thyroid positivity by evaluating TSH and FT4 levels used in this study is shown in Table 3.

\subsection{Hormone Tests}

The vibrant reproductive hormone test includes 13 different reproductive and other related hormones. They are cortisol, parathyroid hormone, estradiol, FSH, LH, progesterone, total testosterone, SHBG, DHEA-S, IGF-I, estriol, estrone,

Table 2. Reference ranges for thyroid markers studied [8].

\begin{tabular}{cc}
\hline Marker & Reference Range \\
\hline TSH & $0.3-4.2 \mathrm{mIU} / \mathrm{L}$ \\
FT4 & $0.9-1.7 \mathrm{ng} / \mathrm{dL}$ \\
Anti- TPO & $<9.0 \mathrm{IU} / \mathrm{mL}$ \\
Anti-Tg & $<4.0 \mathrm{IU} / \mathrm{mL}$ \\
\hline
\end{tabular}

Table 3. Thyroid disease categorization.

\begin{tabular}{ccc}
\hline Disease Condition & TSH & FT4 \\
\hline Hypothyroidism & & \\
Subclinical hypothyroidism & $>4.2 \mathrm{mIU} / \mathrm{L}$ & $0.9-1.7 \mathrm{ng} / \mathrm{dL}$ \\
Overt hypothyroidism & $>4.2 \mathrm{mIU} / \mathrm{L}$ & $<0.9 \mathrm{ng} / \mathrm{dL}$ \\
Hyperthyroidism & & \\
Subclinical hyperthyroidism & $<0.3 \mathrm{mIU} / \mathrm{L}$ & $0.9-1.7 \mathrm{ng} / \mathrm{dL}$ \\
Overt hyperthyroidism & $<0.3 \mathrm{mIU} / \mathrm{L}$ & $>1.7 \mathrm{ng} / \mathrm{dL}$ \\
\hline
\end{tabular}


prolactin. The Elecsys Cortisol II immunoassay (Roche Diagnostics, Indianapolis, IN, USA) is used to measure serum cortisol levels. The Elecsys assay (Roche Diagnostics, Indianapolis, IN, USA) for determining intact Parathyroid hormone employs a sandwich test principle in which a biotinylated monoclonal antibody reacts with the $\mathrm{N}$-terminal fragment and a monoclonal antibody labeled with a ruthenium complex reacts with the C-terminal fragment. The Elecsys Estradiol III assay (Roche Diagnostics, Indianapolis, IN, USA) employs a competitive test principle using two monoclonal antibodies specifically directed against $17 \beta$-estradiol. The Elecsys FSH assay (Roche Diagnostics, Indianapolis, IN, USA) employs two different monoclonal antibodies specifically directed against human FSH. Similarly, the Elecsys LH assay (Roche Diagnostics, Indianapolis, IN, USA) employs two monoclonal antibodies specifically directed against human LH. The Elecsys Testosterone II assay (Roche Diagnostics, Indianapolis, IN, USA) is based on a monoclonal antibody specifically directed against testosterone. The Elecsys SHBG assay (Roche Diagnostics, Indianapolis, IN, USA) employs two monoclonal antibodies specifically directed against human SHBG. The Elecsys DHEA-S assay (Roche Diagnostics, Indianapolis, IN, USA) is based on a monoclonal antibody specifically directed against DHEA-S. An enzyme-labeled chemiluminescent immunometric assay is used to detect human IGF-I. The Elecsys Prolactin II assay (Roche Diagnostics, Indianapolis, IN, USA) employs two monoclonal antibodies specifically directed against human prolactin.

\subsection{Patient and Public Involvement}

This study does not include any patient or public involvement and is based on retrospective analysis of de-identified laboratory data. IRB exemption (work order \#1-1098539-1, July 2018) was determined by the Western Institutional Review Board (WIRB) for Vibrant America Biorepository to use de-linked and de-identified remnant human specimen and medical data for research purposes.

\subsection{Statistical Analysis}

The retrospective analysis on clinical data from de-identified subjects was performed via Java for Windows version 1.8.161 and $\mathrm{R}$ for Windows version 3.5.0. Data were expressed as mean \pm standard deviation (SD) when the distribution was Gaussian. Pearson's Chi-squared test was used when the observed count is $<5$ to evaluate the association between the presence of clinical variables evaluated. $\mathrm{P}$ value $<0.05$ was considered statistically significant.

\section{Results}

\subsection{Reproductive and Other Related Hormones in Women with Thyroid Alterations}

A total of 10,626 women between age 15 - 49 were tested for thyroid hormones and antibodies. The clinical information on majority of these patients were provided by physicians as ICD-10-CM (International Classification of Diseases, 
Tenth Revision, Clinical Modification) codes. The percentage distribution of the top 20 ICD-10-CM codes reported is listed in Table 4. The top two ICD codes were fatigue and Vitamin D deficiency respectively.

Next, the hormone levels of 13 reproductive and other related hormones were simultaneously measured to evaluate any abnormalities in the same cohort. A detailed analysis of the total 13 hormones and their hormonal and antibody level fluctuations are reported in Tables S1-S4 in supplementary material. Details on hormones, which were significantly altered in women with hypothyroidism and hyperthyroidism are shown in Table 5 while Table 6 provides information on elevated/reduced hormones in subjects with elevated anti-TPO and anti-Tg (The total subjects that performed each test was different in each group which is reflected by different denominators).

As shown in Figure 1, cortisol hormone was elevated in women with hypothyroidism while SHBG and DHEA.S levels were significantly reduced compared to the controls (euthyroid subjects). Hyperthyroid women showed elevated levels of total testosterone and SHBG. Women seropositive for anti-TPO had elevated levels of total testosterone and reduced levels of cortisol while women

Table 4. Top 20 physician-reported ICD-10-CM codes distribution for women with thyroid alterations.

\begin{tabular}{|c|c|c|c|c|c|}
\hline ICD-10-CM & Description & Hypothyroidism (\%) & Hyperthyroidism (\%) & Anti-TPO (\%) & Anti-Tg (\%) \\
\hline R5383 & Other fatigue & 55.8 & 55.2 & 58.4 & 59.9 \\
\hline E559 & Vitamin D deficiency, unspecified & 32.2 & 26.2 & 26.9 & 28.2 \\
\hline E039 & Hypothyroidism, unspecified & 24 & 39.3 & 15.6 & 15.8 \\
\hline M2550 & Pain in unspecified joint & 17.4 & 8.3 & 12.9 & 13.8 \\
\hline E349 & Endocrine disorder, unspecified & 12.6 & 19.3 & 13.7 & 12.3 \\
\hline K5900 & Constipation, unspecified & 7.9 & 9 & 8.2 & 9.4 \\
\hline E782 & Mixed hyperlipidemia & 5.7 & 4.8 & 4.3 & 4.7 \\
\hline E785 & Hyperlipidemia, unspecified & 4.7 & 6.2 & 4.3 & 4.7 \\
\hline R638 & Other symptoms and signs concerning food and fluid intake & 6.3 & 2.8 & 6.8 & 7 \\
\hline R5382 & Chronic fatigue, unspecified & 7.3 & 6.2 & 8.2 & 7.1 \\
\hline $\mathrm{Z} 0000$ & $\begin{array}{l}\text { Encounter for general adult medical examination } \\
\text { without abnormal findings }\end{array}$ & 5.7 & 5.5 & 6.7 & 6.2 \\
\hline E079 & Disorder of thyroid, unspecified & 6.6 & 9 & 6.2 & 5.9 \\
\hline F419 & Anxiety disorder, unspecified & 8.2 & 6.2 & 7.1 & 7.4 \\
\hline I10 & Essential (primary) hypertension & 4.1 & 2.1 & 2.1 & 2.4 \\
\hline K909 & Intestinal malabsorption, unspecified & 6 & 4.1 & 5.3 & 5.8 \\
\hline G4700 & Insomnia, unspecified & 8.5 & 2.8 & 6.3 & 6 \\
\hline D539 & Nutritional anemia, unspecified & 3.2 & 6.2 & 4.7 & 4.7 \\
\hline N926 & Irregular menstruation, unspecified & 4.7 & 1.4 & 7.1 & 7 \\
\hline R7989 & Other specified abnormal findings of blood chemistry & 4.4 & 5.5 & 4.6 & 4.5 \\
\hline Z79899 & Other long term (current) drug therapy & 3.8 & 9 & 4.5 & 4.8 \\
\hline
\end{tabular}


Table 5. Reproductive hormonal changes in women with hypothyroidism and hyperthyroidism.

\begin{tabular}{ccccc}
\hline & \multicolumn{2}{c}{ Hypothyroidism } & \multicolumn{2}{c}{ Hyperthyroidism } \\
\cline { 2 - 5 } Cortisol & $\uparrow$ & Range & $\%$ & Range \\
\cline { 2 - 5 } & $\uparrow 103 / 578(17.8 \%)$ & $17.5-46.0$ & $\rightarrow$ & - \\
\hline Total Testosterone & $\rightarrow$ & - & $\uparrow 49 / 279(17.6 \%)$ & $49.0-460.1$ \\
SHBG & $\downarrow 32 / 573(5.6 \%)$ & $6.5-24.2$ & $\uparrow 95 / 266(35.7 \%)$ & $122.1-643$ \\
DHEA-S & $\downarrow 63 / 579(10.9 \%)$ & $0.1-145.9$ & $\rightarrow$ & - \\
\hline
\end{tabular}

$\uparrow$-Increased; $\downarrow$-Reduced; $\rightarrow$-No Change; Units: Cortisol- $\mu$ g/dL; Parathyroid Hormone-pg/mL; Total Testosterone-ng/dL; SHBG-nmol/L; DHEA-S- $\mu \mathrm{g} / \mathrm{dL}$; IGF-I-ng/mL.

Table 6. Reproductive hormonal changes in women with elevated anti-TPO and anti-Tg.

\begin{tabular}{ccccc}
\hline & \multicolumn{2}{c}{ Anti-TPO+ } & \multicolumn{2}{c}{ Anti-Tg+ } \\
\cline { 2 - 5 } & $\%$ & Range & $\%$ & Range \\
\hline Cortisol & $\downarrow 980 / 7618(12.9 \%)$ & $0.4-6.14$ & $\rightarrow$ & - \\
Parathyroid Hormone & $\rightarrow$ & - & $\downarrow 68 / 5331(1.3 \%)$ & $0-14.48$ \\
Testosterone & $\uparrow 1460 / 7873(11.3 \%)$ & $40.87-1478.0$ & $\rightarrow$ & - \\
\hline
\end{tabular}

$\uparrow$-Increased; $\downarrow$-Reduced; $\rightarrow$-No Change; Units: Cortisol- $\mu \mathrm{g} / \mathrm{dL}$; Parathyroid Hormone-pg/mL; Total Testosterone-ng/dL.

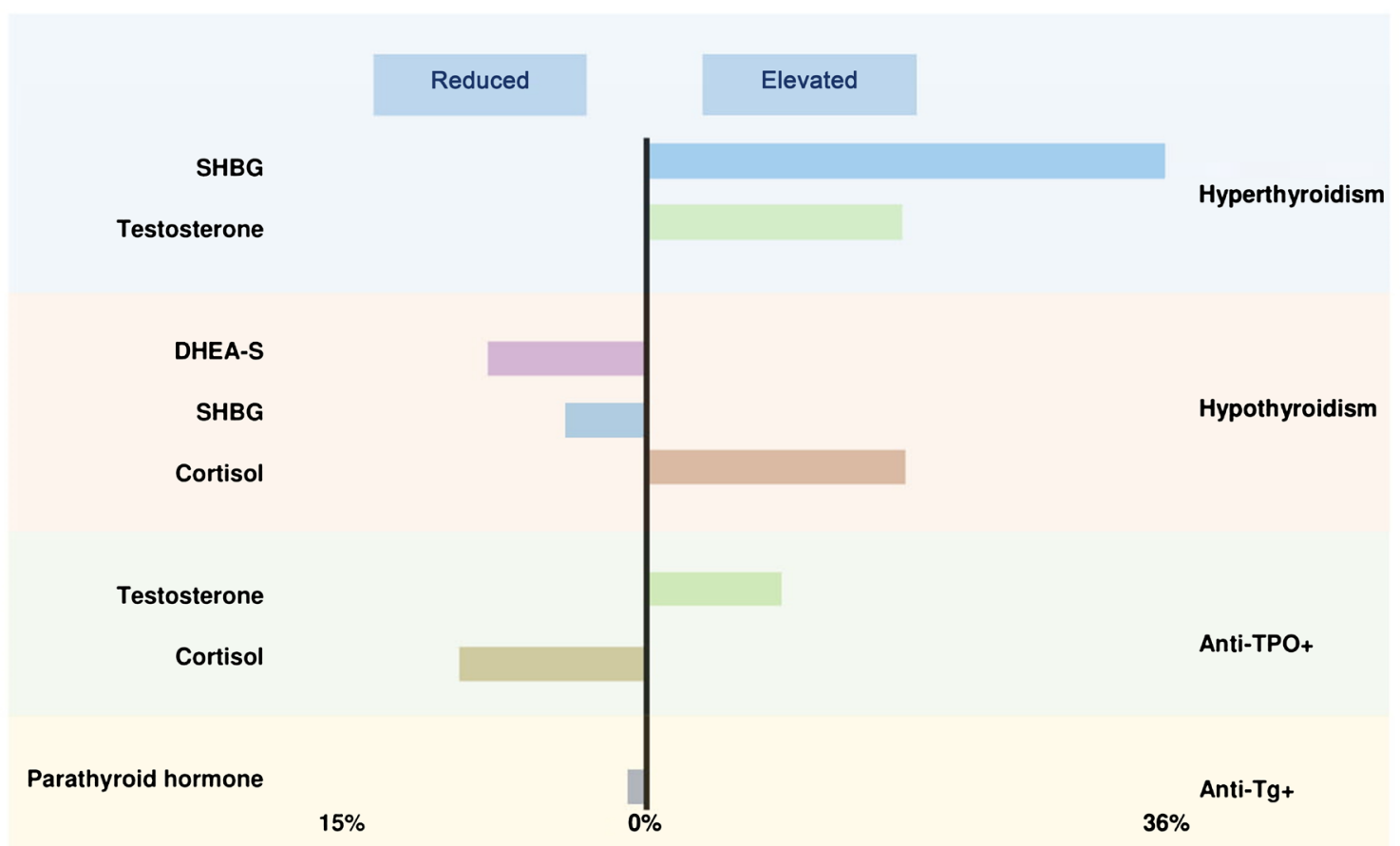

Figure 1. Hormonal changes in female with different thyroid disparities. Significantly altered hormones are only shown in the figure. The percentage of reduction (on the left) or increase (on the right) are shown in comparison to the control groups in each thyroid disorder.

seropositive for anti-Tg reduced levels of parathyroid hormone.

Next, we sought to evaluate the effect of age on reproductive and other related 
hormones in women with thyroid disorder. Table 7 provides the details of the hormones that showed a significant alteration in each age category (refer Tables S5-S16 for full analysis including all 13 hormones).

Age 15 - 25 group only had elevated levels of cortisol and total testosterone respectively in women with hypothyroidism and anti-TPO seropositivity. Age 26 - 35 group only had reduced levels of total testosterone in women with hyperthyroidism. The age group $36-49$ is the most affected group. Hypothyroid women in that age group had elevated levels of cortisol hormone with reduced levels of SHBG, DHEA-S and IGF-I. In contrast, hyperthyroid women in that age group showed elevated levels of total testosterone and SHBG. In addition, women seropositive for anti-TPO had elevated levels of DHEA-S.

\subsection{Reproductive and Other Related Hormones in Men with Thyroid Alterations}

A total of 4417 men between age 15 - 49 were tested for thyroid hormones and autoantibodies. Similarly, The clinical information on majority of these patients were provided by physicians as ICD-10-CM codes. The percentage distribution of the top 20 ICD-10-CM codes reported is listed in Table 8. The top two ICD codes were fatigue and Vitamin $\mathrm{D}$ deficiency respectively.

Table 7. The effect of age on reproductive and other related hormones in women with thyroid disparity.

\begin{tabular}{|c|c|c|c|c|c|c|}
\hline & Cortisol & Parathyroid hormone & Testosterone & SHBG & DHEA-S & IGF-I \\
\hline \multicolumn{7}{|l|}{$\underline{15-25}$} \\
\hline Hypothyroidism & $\uparrow 33 / 88(37.5 \%)$ & $\rightarrow$ & $\rightarrow$ & $\rightarrow$ & $\rightarrow$ & $\rightarrow$ \\
\hline Hyperthyroidism & $\rightarrow$ & $\rightarrow$ & $\rightarrow$ & $\rightarrow$ & $\rightarrow$ & $\rightarrow$ \\
\hline Anti-TPO+ & $\rightarrow$ & $\rightarrow$ & $\uparrow 143 / 1043(13.7 \%)$ & $\rightarrow$ & $\rightarrow$ & $\rightarrow$ \\
\hline Anti-Tg+ & $\rightarrow$ & $\rightarrow$ & $\rightarrow$ & $\rightarrow$ & $\rightarrow$ & $\rightarrow$ \\
\hline \multicolumn{7}{|l|}{$\underline{26-35}$} \\
\hline Hypothyroidism & $\rightarrow$ & $\rightarrow$ & $\rightarrow$ & $\rightarrow$ & $\rightarrow$ & $\rightarrow$ \\
\hline Hyperthyroidism & $\rightarrow$ & $\rightarrow$ & $\downarrow_{4 / 52}(7.7 \%)$ & $\rightarrow$ & $\rightarrow$ & $\rightarrow$ \\
\hline Anti-TPO+ & $\rightarrow$ & $\rightarrow$ & $\rightarrow$ & $\rightarrow$ & $\rightarrow$ & $\rightarrow$ \\
\hline Anti-Tg+ & $\rightarrow$ & $\rightarrow$ & $\rightarrow$ & $\rightarrow$ & $\rightarrow$ & $\rightarrow$ \\
\hline \multicolumn{7}{|l|}{$\underline{36-49}$} \\
\hline Hypothyroidism & $\uparrow 44 / 347$ (12.7\%) & $\rightarrow$ & $\rightarrow$ & $\downarrow_{20 / 347(5.8 \%)}$ & $\downarrow_{35 / 347(10.1 \%)}$ & $\downarrow 10 / 122(7.7 \%)$ \\
\hline Hyperthyroidism & $\rightarrow$ & $\rightarrow$ & $\uparrow 40 / 205(19.5 \%)$ & $\uparrow 70 / 194(36.1 \%)$ & $\rightarrow$ & $\rightarrow$ \\
\hline Anti-TPO+ & $\rightarrow$ & $\rightarrow$ & $\rightarrow$ & $\rightarrow$ & $\uparrow 247 / 4504(5.5 \%)$ & $\rightarrow$ \\
\hline Anti-Tg+ & $\rightarrow$ & $\rightarrow$ & $\rightarrow$ & $\rightarrow$ & $\rightarrow$ & $\rightarrow$ \\
\hline
\end{tabular}

$\uparrow$-Increased; $\downarrow$-Reduced; $\rightarrow$-No Change; Units: Cortisol- $\mu$ g/dL; Parathyroid Hormone-pg/mL; Total Testosterone-ng/dL; SHBG-nmol/L; DHEA-S- $\mu \mathrm{g} / \mathrm{dL}$; IGF-I-ng/mL. 
Table 8. Top 20 physician-reported ICD-10-CM codes distribution for men with thyroid alterations.

\begin{tabular}{|c|c|c|c|c|c|}
\hline ICD-10-CM & Description & Hypothyroidism (\%) & Hyperthyroidism (\%) & Anti-TPO (\%) & Anti-Tg (\%) \\
\hline R5383 & Other fatigue & 60.8 & 65 & 60.5 & 61.1 \\
\hline E559 & Vitamin D deficiency, unspecified & 34.2 & 45 & 33.4 & 35.5 \\
\hline E039 & Hypothyroidism, unspecified & 22.5 & 25 & 13.4 & 13.7 \\
\hline E291 & Testicular hypofunction & 16.7 & 15 & 17.1 & 18.1 \\
\hline M2550 & Pain in unspecified joint & 11.7 & 20 & 17.8 & 18.3 \\
\hline E349 & Endocrine disorder, unspecified & 11.7 & 10 & 10.4 & 10.3 \\
\hline E785 & Hyperlipidemia, unspecified & 10.8 & 15 & 8.2 & 8.2 \\
\hline R638 & Other symptoms and signs concerning food and fluid intake & 10.8 & 5 & 9 & 9.8 \\
\hline R6882 & Decreased libido & 10 & 5 & 9.8 & 9.4 \\
\hline R5381 & Other malaise & 9.2 & 5 & 4.7 & 4.8 \\
\hline E663 & Overweight & 6.7 & 0 & 4.1 & 3.8 \\
\hline R5382 & Chronic fatigue, unspecified & 5.8 & 5 & 5.6 & 5.7 \\
\hline $\mathrm{I} 10$ & Essential (primary) hypertension & 5 & 0 & 6.9 & 6.7 \\
\hline D539 & Nutritional anemia, unspecified & 5 & 10 & 4.9 & 5.2 \\
\hline G4700 & Insomnia, unspecified & 5 & 0 & 6.1 & 6.3 \\
\hline K909 & Intestinal malabsorption, unspecified & 5 & 20 & 3.8 & 4.3 \\
\hline E782 & Mixed hyperlipidemia & 4.2 & 5 & 6.9 & 7 \\
\hline Z79899 & Other long term (current) drug therapy & 4.2 & 10 & 4.9 & 5.2 \\
\hline R7989 & Other specified abnormal findings of blood chemistry & 4.2 & 5 & 4.6 & 4.8 \\
\hline E119 & Type 2 diabetes mellitus without complications & 3.3 & 0 & 3.3 & 3 \\
\hline
\end{tabular}

The hormone levels of 13 reproductive hormones were simultaneously measured to evaluate any abnormalities in the same cohort. A detailed analysis of the total 13 hormones and their hormonal and antibody level fluctuations are reported in Tables S17-S20 in supplementary material. Details on hormones, which were significantly altered in men with hypothyroidism and hyperthyroidism are shown in Table 9 while Table 10 provides information on altered hormones in subjects with elevated anti-TPO and anti-Tg (The total subjects that performed each test was different in each group which is reflected by different denominators).

As shown in Figure 2, IGF-I was elevated in men with hypothyroidism compared to the controls (euthyroid subjects). Hyperthyroid men showed elevated levels of estradiol, FSH, LH and DHEA-S. Men seropositive for anti-TPO had elevated levels of SHBG while men seropositive for anti-Tg had reduced levels of progesterone.

Next, we evaluated the effect of age on reproductive and other related hormones in men with thyroid disorders. Table 11 provides the details of the hormones that showed a significant alteration in each age category (refer Tables S21-S32 for full analysis including all 13 hormones). 
Table 9. Reproductive hormonal changes in men with hypothyroidism and hyperthyroidism.

\begin{tabular}{ccccc}
\hline & \multicolumn{2}{c}{ Hypothyroidism } & \multicolumn{2}{c}{ Hyperthyroidism } \\
\cline { 2 - 5 } & $\%$ & Range & $\%$ & Range \\
\hline SHBG & $\rightarrow$ & - & $\uparrow 14 / 37(37.8 \%)$ & $56.6-189.5$ \\
DHEA-S & $\rightarrow$ & - & $\downarrow 6 / 35(17.1 \%)$ & $23.8-153.7$ \\
IGF-I & $\downarrow 8 / 98(8.2 \%)$ & $15.5-145.0$ & $\rightarrow$ & - \\
Estradiol & $\rightarrow$ & - & $\uparrow 3 / 34(8.8 \%)$ & $61.6-113.9$ \\
FSH & $\rightarrow$ & - & $\uparrow 3 / 30(10.0 \%)$ & $12.9-121.9$ \\
LH & $\rightarrow$ & - & $\uparrow 6 / 30(20.0 \%)$ & $8.7-61.2$ \\
\hline
\end{tabular}

$\uparrow$-Increased; $\downarrow$-Reduced; $\rightarrow$-No Change; Units: Cortisol- $\mu \mathrm{g} / \mathrm{dL} ; \quad$ Estradiol-pg/mL; FSH-mIU/mL; LH-mIU/mL; Total Testosterone-ng/dL; SHBG-nmol/L; DHEA-S- $\mu$ g/dL; IGF-I-ng/mL, Prolactin-ng/mL.

Table 10. Reproductive hormonal changes in men with elevated anti-TPO and anti-Tg.

\begin{tabular}{ccccc}
\hline & \multicolumn{2}{c}{ Anti-TPO+ } & \multicolumn{2}{c}{ Anti-Tg+ } \\
\cline { 2 - 5 }$\%$ & Range & $\%$ & Range \\
\hline \multirow{2}{*}{ SHBG } & $\uparrow 518 / 3102(16.7 \%)$ & $3.6-16.4$ & $\rightarrow$ & - \\
Progesterone & $\downarrow 657 / 2539(25.9 \%)$ & $0.2-6.3$ & $\downarrow 392 / 1965(19.9 \%)$ & $0.2-17.0$
\end{tabular}

$\uparrow$-Increased; $\downarrow$-Reduced; $\rightarrow$-No Change; Units: Cortisol- $\mu$ g/dL; Parathyroid Hormone-pg/mL; Estradiol-pg/mL; FSH-mIU/mL; Progesterone-ng/mL.

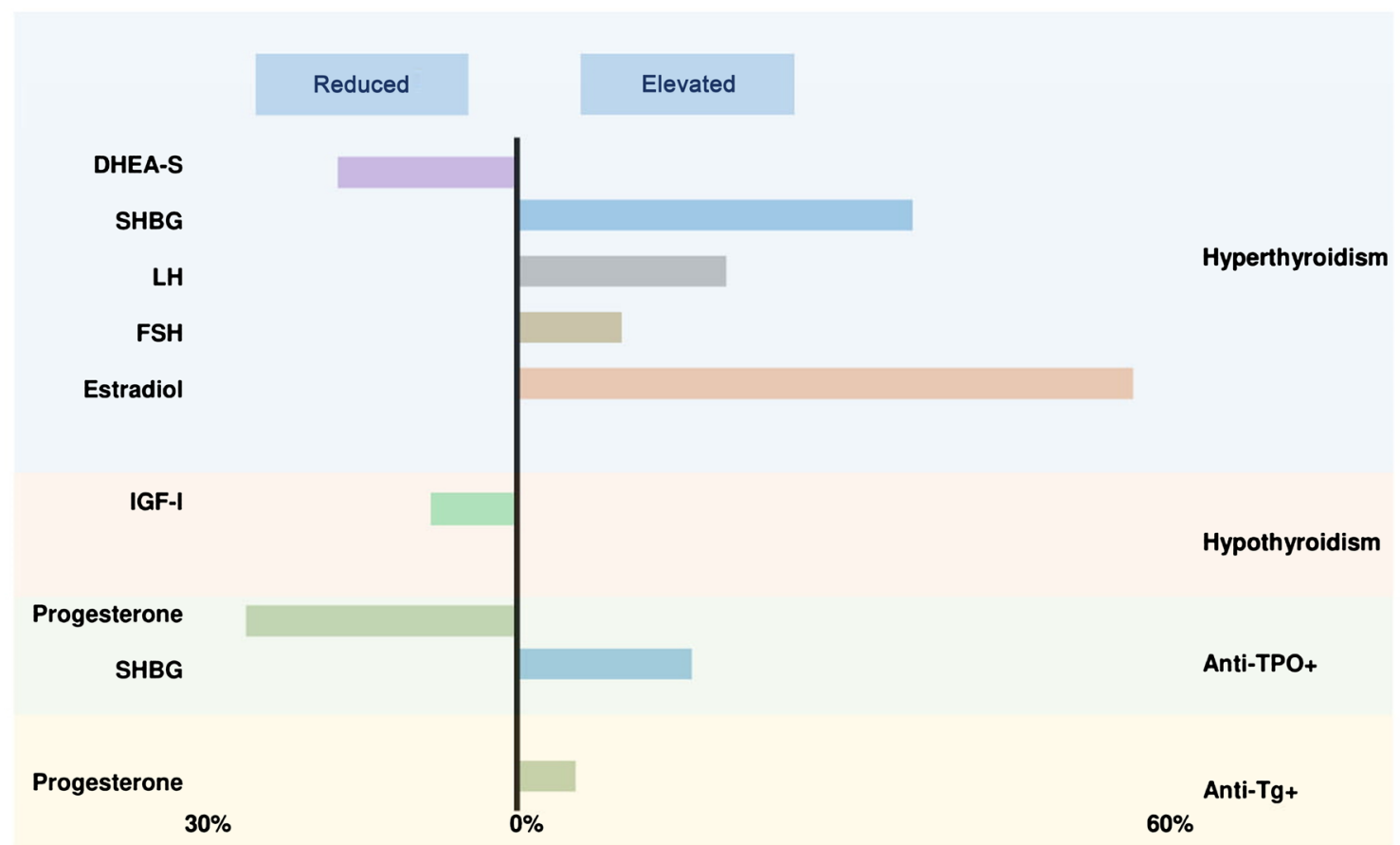

Figure 2. Hormonal changes in male with different thyroid disparities. Significantly altered hormones are only shown in the figure. The percentage of reduction (on the left) or increase (on the right) are shown in comparison to the control groups in each thyroid disorder. 
Table 11. The effect of age on reproductive and other related hormones in men with thyroid disparity.

\begin{tabular}{|c|c|c|c|c|c|c|}
\hline & Testosterone & SHBG & DHEA-S & IGF-I & Estradiol & Progesterone \\
\hline \multicolumn{7}{|l|}{$\underline{15-25}$} \\
\hline Hypothyroidism & $\downarrow 10 / 43(23.3 \%)$ & $\rightarrow$ & $\rightarrow$ & $\downarrow 3 / 20(15.0 \%)$ & $\rightarrow$ & $\rightarrow$ \\
\hline Hyperthyroidism & $\rightarrow$ & $\rightarrow$ & $\rightarrow$ & $\rightarrow$ & $\rightarrow$ & $\rightarrow$ \\
\hline Anti-TPO+ & $\rightarrow$ & $\rightarrow$ & $\rightarrow$ & $\rightarrow$ & $\rightarrow$ & $\uparrow 37 / 351(10.5 \%)$ \\
\hline Anti-Tg+ & $\rightarrow$ & $\rightarrow$ & $\rightarrow$ & $\rightarrow$ & $\rightarrow$ & $\rightarrow$ \\
\hline \multicolumn{7}{|l|}{$\underline{26-35}$} \\
\hline Hypothyroidism & $\rightarrow$ & $\rightarrow$ & $\rightarrow$ & $\rightarrow$ & $\rightarrow$ & $\rightarrow$ \\
\hline Hyperthyroidism & $\rightarrow$ & $\rightarrow$ & $\rightarrow$ & $\rightarrow$ & $\rightarrow$ & $\rightarrow$ \\
\hline Anti-TPO+ & $\rightarrow$ & $\rightarrow$ & $\rightarrow$ & $\rightarrow$ & $\rightarrow$ & $\downarrow 172 / 699(24.6 \%)$ \\
\hline Anti-Tg+ & $\rightarrow$ & $\rightarrow$ & $\rightarrow$ & $\rightarrow$ & $\rightarrow$ & $\rightarrow$ \\
\hline \multicolumn{7}{|l|}{$\underline{36-49}$} \\
\hline Hypothyroidism & $\rightarrow$ & $\rightarrow$ & $\rightarrow$ & $\rightarrow$ & $\rightarrow$ & $\rightarrow$ \\
\hline Hyperthyroidism & $\rightarrow$ & $\uparrow 13 / 28(46.4)$ & $\downarrow 4 / 25(16.0 \%)$ & $\rightarrow$ & $\uparrow 3 / 25(12.0 \%)$ & $\rightarrow$ \\
\hline Anti-TPO+ & $\rightarrow$ & $\uparrow 323 / 1860$ (17.4\%) & $\rightarrow$ & $\rightarrow$ & $\rightarrow$ & $\downarrow 436 / 1489(29.3 \%)$ \\
\hline Anti-Tg+ & $\rightarrow$ & $\rightarrow$ & $\rightarrow$ & $\rightarrow$ & $\rightarrow$ & $\uparrow 55 / 1146(4.8 \%)$ \\
\hline
\end{tabular}

$\uparrow$-Increased; $\downarrow$-Reduced; $\rightarrow$-No Change; Units: Total Testosterone-ng/dL; SHBG-nmol/L; DHEA-S- $\mu \mathrm{g} / \mathrm{dL} ;$ IGF-I-ng/mL; Estradiol-pg/mL; Progesterone$\mathrm{ng} / \mathrm{mL}$.

Age 15 - 25 group only had reduced levels of total testosterone and IGF-1 in hypothyroid subjects and elevated levels of progesterone in seropositive anti-TPO subjects. Age 26 - 35 group only had reduced levels of progesterone in men seropositive for anti-TPO. The age group 36 - 49 is the most affected group. Hyperthyroid men in that age group had elevated levels of SHBG and estradiol hormones with reduced levels of DHEA-S. Men seropositive for anti-TPO had elevated levels of SHBG and reduced levels of progesterone hormone. Men seropositive for anti-Tg had elevated levels of progesterone.

\section{Discussion}

Thyroid alterations are associated with other disorders and closely linked to the diseases that are under the control of the hypothalamus-pituitary-gonadal axis. Sexual and reproductive-related hormones are controlled by the hypothalamus-pituitary-gonadal axis and affect the individuals with derailed thyroid functions. Our study is a comprehensive analysis including the common thyroid subcategories (subclinical/overt hypothyroidism and subclinical/overt hyperthyroidism) and autoantibodies related to AITD separately for 13 sexual and reproductive-related hormones for a large population size of 15,043 including 10,626 women and 4417 men in different reproductive age groups.

In this study, we used the physician reported ICD-10-CM codes to provide the clinical information about the subjects. The ICD-10-CM an internationally rec- 
ognized alphanumeric code, created by the World Health Organization (WHO) for physicians and other healthcare providers to report their diagnosis, symptoms, and procedures specific for each disease, disorder, injury, infection, and symptom. The percentage distribution of the ICD-10-CM could provide clinical information on subjects used in this study. The top ranked ICD-10-CM codes were nonspecific symptoms representing fatigue (ICD-10-CM-R5383) and vitamin D deficiency (ICD-10-CM-E559). Thyroid disease usually contains nonspecific symptoms such as fatigue, hair loss, etc. which are also symptoms of vitamin D deficiency.

Our analysis between thyroid dysfunction and sexual and reproductive-related hormones showed that different thyroid alterations could affect sexual and reproductive-related hormones in different ways. Hyperthyroid women showed elevated levels of SHBG and total testosterone, while hypothyroid women showed reduced levels of SHBG and DHEA-S but elevated levels of cortisol. Hyperthyroid men had significantly elevated levels of SHBG, LH, FSH, estradiol and reduced levels of DHEA-S, while hypothyroid men had reduced levels of IGF-I. An interesting finding is that SHBG levels were in opposite directions in hyperthyroidism and hypothyroidism in women. SHBG is a protein that binds and transports sex hormones such as testosterone, estrogen to target cells. Studies showed that the levels of SHBG could be increased in hyperthyroidism due to the effects of elevated thyroid hormones on its production by the liver, [9] which was also reflected by our results. Several studies also showed a positive correlation between SHBG binding capacity and FT4 and FT3 levels in hyperthyroid patients. [10] The increase in the affinity of SHBG to testosterone in hyperthyroid subjects could reduce the metabolic clearance rate of testosterone thus, increasing the total serum testosterone levels that were agreeing with our results for hyperthyroid women. However, the underlying mechanism of the effect of hypothyroidism on SHBG is still under review, but our results show that the effect is opposite to that in hyperthyroidism in women [11]. Some studies have shown subnormal to normal levels of SHBG in hypothyroidism [12]. Our data followed the same pattern for men, where hyperthyroidism showed elevated levels of SHBG while hypothyroidism showed normal levels [13]. Since the SHBG level has least effect on reducing the testosterone levels in hypothyroidism, as suggested by other studies, the primary reason for the reduction may be due to hypogonadotropic hypogonadism, where hypothyroidism impairs the ability of pituitary to respond to GnRH, thus, ultimately reducing the testosterone secretion [14]. Apart from these major hormones Cortisol, DHEA-S and IGF-I have shown significantly different levels in subjects with thyroid disease compared to control subjects. Cortisol is a steroid hormone, produced in the adrenal gland and releases in response to any kind of stress in the body. The elevated levels of cortisol in hypothyroidism in women could be mainly due to the decreased metabolic clearance of cortisol and decrease in the negative feedback of cortisol on the hypothalamic-pituitary-adrenal axis [15]. DHEA-S is an an- 
drostane steroid that is produced by sulfation of DHEA in the adrenal cortex. DHEA-S is one of the precursors of male hormones androstenedione, testosterone and dihydrotestosterone and female hormone estrogens [16]. We found that serum DHEA-S levels were significantly reduced in hypothyroid women. As Tagawa et al. suggested, the reduction in DHEA-S in hypothyroidism may be due to the significant reduction in the activity of the enzyme, cytochrome $\mathrm{P}-450_{\mathrm{SCC}}$ involved in the biosynthesis of all steroidal hormones, including DHEA-S [17]. Our results with DHEA-S in hyperthyroid men showed opposite results compared to previous studies done by other researchers. They suggest that steroidogenesis could be activated by thyroid hormones in hyperthyroidism leading to elevated levels of DHEA-S [17] [18]. However, we found reduced levels of DHEA-S in hyperthyroid men. Estradiol, FSH, LH and Progesterone were not included in the analysis for women since the reference ranges were depending on women hormonal phases. Even though estradiol is predominant in female sex hormone estrogen, it also plays an essential role in male sexual functions [19]. Studies have shown that elevated levels of SHBG in hyperthyroidism could indirectly increase the levels of LH and estradiol [20]. Our results followed the same trend as hyperthyroid men in our study had elevated levels of SHBG, LH and estradiol.

Next, we focused on assessing the sexual and reproductive-related hormone levels in subjects with seropositive thyroid autoantibodies, anti-TPO and anti-Tg. Our results showed that women with anti-TPO positivity had reduced levels of cortisol and elevated levels of total testosterone. Reduced cortisol levels could be attributed to the primary adrenal insufficiency (PAI) or failure to produce adequate levels of cortisol in autoimmune thyroid disease that has been reported in variable occurrences in different countries [21]. In our results, we observed high levels of testosterone in women seropositive for anti-TPO. The prevalence of thyroid autoimmunity has been found to be consistently increased in polycystic ovary syndrome (PCOS) that could lead to increase testosterone levels in women. It is suspected that PCOS and autoimmune thyroid disease may have a pathogenic link since they share similar factors, such as genetic susceptibility and sub inflammation/autoimmunity to the development of the disease [22]. In addition, we found higher levels of SHBG and low levels of progesterone in anti-TPO positive men, while reduced levels of parathyroid hormone and elevated levels of progesterone in women and men with anti-Tg positivity respectively.

Finally, we grouped the thyroid subcategories of men and women into smaller sub-groups based on their age to evaluate the effect of age on their reproductive and other related hormones. In both genders, age groups $15-25$ and $26-35$ did not show any major effect on reproductive and other related hormones. However, the age group 36 - 49 showed the most significant effect confirming that age has a significant effect on reproductive hormonal changes in thyroid diseased subjects.

One of the limitations in our study was the inability to account for subjects with other thyroid diseases such as central hypothyroidism. We did not account 
for these secondary thyroid diseases since these disorders are very rare compared to primary thyroid disease. In addition, our study did not account for the smoking history and ethnicity because this information was not available in the data source.

\section{Conclusion}

In conclusion, our comprehensive study showed that different thyroid alterations affect differently on both male and female reproductive health, especially in the age group 36 - 49. Hence, frequent testing and follow-up routines should be considered on reproductive and other related hormones specially, if an individual in the age group 36 - 49 is susceptible to thyroid disease.

\section{Ethics Approval and Consent to Participate}

This study is based on retrospective analysis of de-identified laboratory data, hence was exempted from formal ethical reviews by WIRB (\#1-1098539-1). The data and materials in this manuscript have not been published elsewhere and are not under consideration by another journal.

\section{Availability of Data and Material}

The data sets used and/or analyzed during the current study are available from the corresponding author on reasonable request.

\section{Author Contributions}

TS, HK, KK, and TW performed the research. TS, HK, JJ, and VJ designed the study. TS, HK, KB, QS and VR analyzed the data. TS and HK wrote the article.

\section{Acknowledgement}

We acknowledge Vibrant America LLC for supporting this research.

\section{Competing Interests}

Siriwardhane is an employee of Vibrant America LLC. Krishna, Ranganathan, Song, Jayaraman, Wang, Bei, Rajasekaran, Krishnamurthy, are employees of Vibrant Sciences LLC, CA, USA.

\section{Conflicts of Interest}

The authors declare no conflicts of interest regarding the publication of this paper.

\section{References}

[1] Sarrel, P.M. (1990) Ovarian Hormones and the Circulation. Maturitas, 12, 287-298. https://doi.org/10.1016/0378-5122(90)90008-T

[2] Katib, A. (2015) Mechanisms Linking Obesity to Male Infertility. Central European Journal of Urology, 68, 79-85. https://doi.org/10.5173/ceju.2015.01.435

[3] Jefferys, A., Vanderpump, M. and Yasmin, E. (2015) Thyroid Dysfunction and Re- 
productive Health. The Obstetrician \& Gynaecologist, 17, 39-45.

https://doi.org/10.1111/tog.12161

[4] Kumar, A., Shekhar, S. and Dhole, B. (2014) Thyroid and Male Reproduction. Indian Journal of Endocrinology and Metabolism, 18, 23-31. https://doi.org/10.4103/2230-8210.126523

[5] Seungdamrong, A., et al. (2017) Preconceptional Antithyroid Peroxidase Antibodies, But Not Thyroid-Stimulating Hormone, Are Associated with Decreased Live Birth Rates in Infertile Women. Fertility and Sterility, 108, 843-850.

https://doi.org/10.1016/j.fertnstert.2017.08.026

[6] Mehran, L., et al. (2013) Management of Thyroid Peroxidase Antibody Euthyroid Women in Pregnancy: Comparison of the American Thyroid Association and the Endocrine Society Guidelines. Journal of Thyroid Research, 2013, Article ID: 542692. https://doi.org/10.1155/2013/542692

[7] Trummer, H., et al. (2001) Thyroid Hormones and Thyroid Antibodies in Infertile Males. Fertility and Sterility, 76, 254-257. https://doi.org/10.1016/S0015-0282(01)01875-1

[8] Mayo Clinic Laboratories. 1995-2019. http://mayocliniclabs.com/test-catalog

[9] Selva, D.M. and Hammond, G.L. (2009) Thyroid Hormones Act Indirectly to Increase Sex Hormone-Binding Globulin Production by Liver via Hepatocyte Nuclear Factor-4a. Journal of Molecular Endocrinology, 43, 19-27. https://doi.org/10.1677/JME-09-0025

[10] Pugeat, M., et al. (1996) Clinical Utility of Sex Hormone-Binding Globulin Measurement. Hormone Research, 45, 148-155. https://doi.org/10.1159/000184778

[11] Brenta, G., et al. (1999) Variations of Sex Hormone-Binding Globulin in Thyroid Dysfunction. Thyroid, 9, 273-277. https://doi.org/10.1089/thy.1999.9.273

[12] Hampl, R., et al. (2003) Interpretation of Sex Hormone-Binding Globulin Levels in Thyroid Disorders. Thyroid, 13, 755-760. https://doi.org/10.1089/105072503768499644

[13] Foldes, J., et al. (1990) The Measurement of the Serum Sex-Hormone Binding Globulin in Various Thyroid Diseases. Acta Medica Hungarica, 47, 81-90.

[14] Meikle, A.W. (2004) The Interrelationships between Thyroid Dysfunction and Hypogonadism in Men and Boys. Thyroid, 14, S17-S25.

https://doi.org/10.1089/105072504323024552

[15] Iranmanesh, A., et al. (1990) Dynamics of 24-Hour Endogenous Cortisol Secretion and Clearance in Primary Hypothyroidism Assessed before and after Partial Thyroid Hormone Replacement. The Journal of Clinical Endocrinology \& Metabolism, 70, 155-161. https://doi.org/10.1210/jcem-70-1-155

[16] Longcope, C. (1996) Dehydroepiandrosterone Metabolism. Journal of Endocrinology, 150, S125-S127.

[17] Tagawa, N., et al. (2000) Serum Dehydroepiandrosterone, Dehydroepiandrosterone Sulfate, and Pregnenolone Sulfate Concentrations in Patients with Hyperthyroidism and Hypothyroidism. Clinical Chemistry, 46, 523-528.

[18] Yamakita, N., et al. (2001) Dehydroepiandrosterone Sulphate Is Increased and Dehydroepiandrosterone-Response to Corticotrophin-Releasing Hormone Is Decreased in the Hyperthyroid State Compared with the Euthyroid State. Clinical Endocrinology, 55, 797-803. https://doi.org/10.1046/j.1365-2265.2001.01420.x

[19] Schulster, M., Bernie, A.M. and Ramasamy, R. (2016) The Role of Estradiol in Male 
Reproductive Function. Asian Journal of Andrology, 18, 435-440. https://doi.org/10.4103/1008-682X.173932

[20] Sanyal, T., et al. (2012) Gynaecomastia as the Initial Presentation of Thyrotoxicosis. Indian Journal of Endocrinology and Metabolism, 16, S352-S353.

[21] Yamamoto, T. (2015) Comorbid Latent Adrenal Insufficiency with Autoimmune Thyroid Disease. European Thyroid Journal, 4, 201-206. https://doi.org/10.1159/000433532

[22] Arduc, A., et al. (2015) High Prevalence of Hashimoto's Thyroiditis in Patients with Polycystic Ovary Syndrome: Does the Imbalance between Estradiol and Progesterone Play a Role? Endocrine Research, 40, 204-210.

https://doi.org/10.3109/07435800.2015.1015730 


\section{Supplementary Material}

Table S1. Age group 15 - 49. Reproductive hormonal changes in women with hypothyroidism. The significance was not considered when the total number of subjects tested for each hormonal group is $\leq 10$.

\begin{tabular}{|c|c|c|c|c|c|c|c|c|c|c|}
\hline & \multicolumn{4}{|c|}{ Hypothyroidism $(\mathrm{n}=641)$} & \multicolumn{6}{|c|}{ Thyroid Negative $(\mathrm{n}=9217)$} \\
\hline & Reduced & $\begin{array}{c}\text { Range } \\
\text { (reduced) }\end{array}$ & Elevated & $\begin{array}{c}\text { Range } \\
\text { (elevated) }\end{array}$ & Reduced & $\begin{array}{c}\text { Range } \\
\text { (reduced) }\end{array}$ & $\begin{array}{l}\text { P value } \\
\text { (reduced) }\end{array}$ & Elevated & $\begin{array}{c}\text { Range } \\
\text { (elevated) }\end{array}$ & $\begin{array}{l}\text { P value } \\
\text { (elevated) }\end{array}$ \\
\hline Cortisol & $\begin{array}{c}60 / 578 \\
(10.4 \%)\end{array}$ & $0.558-6.12$ & $\begin{array}{l}103 / 578 \\
(17.8 \%)\end{array}$ & $17.04-46$ & $\begin{array}{c}1036 / 8256 \\
(12.5 \%)\end{array}$ & $0.365-6.14$ & 0.1434 & $\begin{array}{c}983 / 8256 \\
(11.9 \%)\end{array}$ & $12.1-55.37$ & 0 \\
\hline $\begin{array}{c}\text { Parathyroid } \\
\text { Hormone }\end{array}$ & $\begin{array}{l}2 / 495 \\
(0.4 \%)\end{array}$ & $\begin{array}{c}13.74- \\
14.43\end{array}$ & $\begin{array}{l}32 / 495 \\
(6.5 \%)\end{array}$ & $65.5-498.9$ & $\begin{array}{c}74 / 7056 \\
(1 \%)\end{array}$ & $5.5-14.48$ & 0.2397 & $\begin{array}{c}357 / 7056 \\
(5.1 \%)\end{array}$ & $65.5-200.1$ & 0.207 \\
\hline Estradiol & N/A & N/A & N/A & N/A & $0 / 1(0 \%)$ & N/A & 1 & $0 / 1(0 \%)$ & N/A & 1 \\
\hline FSH & N/A & N/A & N/A & N/A & $0 / 3(0 \%)$ & N/A & 1 & $1 / 3(33.3 \%)$ & $\begin{array}{c}61.09- \\
61.09\end{array}$ & 1 \\
\hline $\mathrm{LH}$ & N/A & N/A & N/A & N/A & $0 / 1(0 \%)$ & N/A & 1 & $0 / 1(0 \%)$ & N/A & 1 \\
\hline Progesterone & N/A & N/A & N/A & N/A & $0 / 1(0 \%)$ & N/A & 1 & $0 / 1(0 \%)$ & N/A & 1 \\
\hline $\begin{array}{c}\text { Total } \\
\text { Testosterone }\end{array}$ & $\begin{array}{l}124 / 582 \\
(21.3 \%)\end{array}$ & $2.5-59.5$ & $\begin{array}{l}40 / 582 \\
(6.9 \%)\end{array}$ & $48.6-523.1$ & $\begin{array}{c}1762 / 8503 \\
(20.7 \%)\end{array}$ & $2.5-159.2$ & 0.7771 & $\begin{array}{c}730 / 8503 \\
(8.6 \%)\end{array}$ & $\begin{array}{c}48.18- \\
1010\end{array}$ & 0.1745 \\
\hline SHBG & $\begin{array}{l}32 / 573 \\
(5.6 \%)\end{array}$ & $6.5-24.2$ & $\begin{array}{l}122 / 573 \\
(21.3 \%)\end{array}$ & $\begin{array}{c}123.1- \\
844.9\end{array}$ & $\begin{array}{c}239 / 8270 \\
(2.9 \%)\end{array}$ & $5.14-24.5$ & 0.0005 & $\begin{array}{c}2117 / 8270 \\
(25.6 \%)\end{array}$ & $\begin{array}{c}122.1- \\
732.3\end{array}$ & 0.0249 \\
\hline DHEA-S & $\begin{array}{c}63 / 579 \\
(10.9 \%)\end{array}$ & $0.1-145.9$ & $\begin{array}{l}34 / 579 \\
(5.9 \%)\end{array}$ & $\begin{array}{c}256.4- \\
641.5\end{array}$ & $\begin{array}{c}678 / 8249 \\
(8.2 \%)\end{array}$ & $3.34-159.3$ & 0.0312 & $\begin{array}{c}634 / 8249 \\
(7.7 \%)\end{array}$ & $\begin{array}{c}256.6- \\
881.1\end{array}$ & 0.1301 \\
\hline IGF-I & $\begin{array}{l}12 / 189 \\
(6.3 \%)\end{array}$ & $35.51-141$ & $\begin{array}{l}9 / 189 \\
(4.8 \%)\end{array}$ & $246,413.83$ & $\begin{array}{c}120 / 3237 \\
(3.7 \%)\end{array}$ & $16.78-203$ & 0.101 & $\begin{array}{c}182 / 3237 \\
(5.6 \%)\end{array}$ & $198,585.18$ & 0.7352 \\
\hline Estriol & $0 / 91(0 \%)$ & N/A & $3 / 91(3.3 \%)$ & $\begin{array}{c}300.7- \\
635.3\end{array}$ & $0 / 1327(0 \%)$ & N/A & 1 & $\begin{array}{c}27 / 1327 \\
(2 \%)\end{array}$ & $\begin{array}{c}351.3- \\
851.8\end{array}$ & 0.4357 \\
\hline Estrone & $\begin{array}{c}15 / 120 \\
(12.5 \%)\end{array}$ & $9.3-16.2$ & $0 / 120(0 \%)$ & N/A & $\begin{array}{c}278 / 1860 \\
(14.9 \%)\end{array}$ & $9-16.5$ & 0.5493 & $\begin{array}{c}43 / 1860 \\
(2.3 \%)\end{array}$ & $\begin{array}{l}186.4- \\
859.52\end{array}$ & 0.1081 \\
\hline Prolactin & $0 / 10(0 \%)$ & N/A & $3 / 10(30 \%)$ & $\begin{array}{c}33.46- \\
35.84\end{array}$ & $4 / 150(2.7 \%)$ & $3.92-4.78$ & 1 & $\begin{array}{l}28 / 150 \\
(18.7 \%)\end{array}$ & $\begin{array}{c}23.51- \\
75.58\end{array}$ & 0.409 \\
\hline
\end{tabular}

Table S2. Age group 15 - 49. Reproductive hormonal changes in women with hyperthyroidism. The significance was not considered when the total number of subjects tested for each hormonal group is $\leq 10$.

\begin{tabular}{|c|c|c|c|c|c|c|c|c|c|c|}
\hline & \multicolumn{4}{|c|}{ Hyperthyroidism $(\mathrm{n}=301)$} & \multicolumn{6}{|c|}{ Thyroid Negative $(\mathrm{n}=9217)$} \\
\hline & Reduced & $\begin{array}{c}\text { Range } \\
\text { (reduced) }\end{array}$ & Elevated & $\begin{array}{c}\text { Range } \\
\text { (elevated) }\end{array}$ & Reduced & $\begin{array}{c}\text { Range } \\
\text { (reduced) }\end{array}$ & $\begin{array}{l}\text { P value } \\
\text { (reduced) }\end{array}$ & Elevated & $\begin{array}{c}\text { Range } \\
\text { (elevated) }\end{array}$ & $\begin{array}{l}\mathrm{P} \text { value } \\
\text { (elevated) }\end{array}$ \\
\hline Cortisol & $\begin{array}{l}29 / 253 \\
(11.5 \%)\end{array}$ & $0.801-6.13$ & $\begin{array}{c}26 / 253 \\
(10.3 \%)\end{array}$ & $\begin{array}{c}12.43- \\
36.47\end{array}$ & $\begin{array}{c}1036 / 8256 \\
(12.5 \%)\end{array}$ & $0.365-6.14$ & 0.6761 & $\begin{array}{c}983 / 8256 \\
(11.9 \%)\end{array}$ & $12.1-55.37$ & 0.4895 \\
\hline $\begin{array}{c}\text { Parathyroid } \\
\text { Hormone }\end{array}$ & $\begin{array}{l}5 / 212 \\
(2.4 \%)\end{array}$ & $12.44-13.8$ & $\begin{array}{l}7 / 212 \\
(3.3 \%)\end{array}$ & $\begin{array}{c}66.14- \\
203.7\end{array}$ & $74 / 7056(1 \%)$ & $5.5-14.48$ & 0.1399 & $\begin{array}{c}357 / 7056 \\
(5.1 \%)\end{array}$ & $65.5-200.1$ & 0.3191 \\
\hline Estradiol & N/A & N/A & N/A & N/A & $0 / 1(0 \%)$ & N/A & 1 & $0 / 1(0 \%)$ & N/A & 1 \\
\hline FSH & N/A & N/A & N/A & N/A & $0 / 3(0 \%)$ & N/A & 1 & $1 / 3(33.3 \%)$ & $\begin{array}{c}61.09- \\
61.09\end{array}$ & 1 \\
\hline LH & N/A & N/A & N/A & N/A & $0 / 1(0 \%)$ & N/A & 1 & $0 / 1(0 \%)$ & N/A & 1 \\
\hline Progesterone & N/A & N/A & N/A & N/A & $0 / 1(0 \%)$ & N/A & 1 & $0 / 1(0 \%)$ & N/A & 1 \\
\hline
\end{tabular}


Continued

\begin{tabular}{|c|c|c|c|c|c|c|c|c|c|c|}
\hline $\begin{array}{c}\text { Total } \\
\text { Testosterone }\end{array}$ & $\begin{array}{c}51 / 279 \\
(18.3 \%)\end{array}$ & $2.62-134.9$ & $\begin{array}{c}49 / 279 \\
(17.6 \%)\end{array}$ & $49,460.10$ & $\begin{array}{c}1762 / 8503 \\
(20.7 \%)\end{array}$ & $2.5-159.2$ & 0.3593 & $\begin{array}{c}730 / 8503 \\
(8.6 \%)\end{array}$ & $\begin{array}{c}48.18- \\
1010\end{array}$ & 0 \\
\hline SHBG & $\begin{array}{l}2 / 266 \\
(0.8 \%)\end{array}$ & $12.3-22.5$ & $\begin{array}{c}95 / 266 \\
(35.7 \%)\end{array}$ & $122.1-643$ & $\begin{array}{c}239 / 8270 \\
(2.9 \%)\end{array}$ & $5.14-24.5$ & 0.0361 & $\begin{array}{c}2117 / 8270 \\
(25.6 \%)\end{array}$ & $\begin{array}{c}122.1- \\
732.3\end{array}$ & 0.0003 \\
\hline DHEA-S & $\begin{array}{l}22 / 267 \\
(8.2 \%)\end{array}$ & $11.9-135.5$ & $\begin{array}{l}23 / 267 \\
(8.6 \%)\end{array}$ & $\begin{array}{c}269.1- \\
579.1\end{array}$ & $\begin{array}{c}678 / 8249 \\
(8.2 \%)\end{array}$ & $3.34-159.3$ & 1 & $\begin{array}{c}634 / 8249 \\
(7.7 \%)\end{array}$ & $\begin{array}{c}256.6- \\
881.1\end{array}$ & 0.6577 \\
\hline IGF-I & $4 / 101(4 \%)$ & $\begin{array}{c}55.56- \\
96.63\end{array}$ & $\begin{array}{l}8 / 101 \\
(7.9 \%)\end{array}$ & $210,554.80$ & $\begin{array}{c}120 / 3237 \\
(3.7 \%)\end{array}$ & $16.78-203$ & 0.7888 & $\begin{array}{c}182 / 3237 \\
(5.6 \%)\end{array}$ & 198,585 & 0.4451 \\
\hline Estriol & $0 / 26(0 \%)$ & N/A & $0 / 26(0 \%)$ & N/A & $0 / 1327(0 \%)$ & N/A & 1 & $\begin{array}{c}27 / 1327 \\
(2 \%)\end{array}$ & $\begin{array}{c}351.3- \\
851.8\end{array}$ & 1 \\
\hline Estrone & $\begin{array}{c}8 / 59 \\
(13.6 \%)\end{array}$ & $11.1-16.2$ & $\begin{array}{c}1 / 59 \\
(1.7 \%)\end{array}$ & $\begin{array}{c}493.4- \\
493.4\end{array}$ & $\begin{array}{c}278 / 1860 \\
(14.9 \%)\end{array}$ & $9-16.5$ & 0.9133 & $\begin{array}{c}43 / 1860 \\
(2.3 \%)\end{array}$ & $\begin{array}{l}186.4- \\
859.52\end{array}$ & 1 \\
\hline Prolactin & $0 / 4(0 \%)$ & N/A & $0 / 4(0 \%)$ & N/A & $4 / 150(2.7 \%)$ & $3.92-4.78$ & 1 & $\begin{array}{c}28 / 150 \\
(18.7 \%)\end{array}$ & $\begin{array}{c}23.51- \\
75.58\end{array}$ & 1 \\
\hline
\end{tabular}

Table S3. Age group 15 - 49. Reproductive hormonal changes in women with positive anti-TPO. The significance was not considered when the total number of subjects tested for each hormonal group is $\leq 10$.

\begin{tabular}{|c|c|c|c|c|c|c|c|c|c|c|}
\hline & \multicolumn{4}{|c|}{ Anti-TPO+ $(\mathrm{n}=8592)$} & \multicolumn{6}{|c|}{ Anti-TPO- $(\mathrm{n}=1950)$} \\
\hline & Reduced & $\begin{array}{c}\text { Range } \\
\text { (reduced) }\end{array}$ & Elevated & $\begin{array}{c}\text { Range } \\
\text { (elevated) }\end{array}$ & Reduced & $\begin{array}{c}\text { Range } \\
\text { (reduced) }\end{array}$ & $\begin{array}{l}\text { P value } \\
\text { (reduced) }\end{array}$ & Elevated & $\begin{array}{c}\text { Range } \\
\text { (elevated) }\end{array}$ & $\begin{array}{l}\text { P value } \\
\text { (elevated) }\end{array}$ \\
\hline Cortisol & $\begin{array}{c}980 / 7618 \\
(12.9 \%)\end{array}$ & $0.365-6.14$ & $\begin{array}{c}936 / 7618 \\
(12.3 \%)\end{array}$ & $12.1-55.37$ & $\begin{array}{c}191 / 1792 \\
(10.7 \%)\end{array}$ & $0.7-6.1$ & 0.0122 & $\begin{array}{c}208 / 1792 \\
(11.6 \%)\end{array}$ & $12.5-47.1$ & 0.4521 \\
\hline $\begin{array}{l}\text { Parathyroid } \\
\text { Hormone }\end{array}$ & $\begin{array}{c}68 / 6436 \\
(1.1 \%)\end{array}$ & $7-14.48$ & $\begin{array}{c}312 / 6436 \\
(4.8 \%)\end{array}$ & $65.5-268.6$ & $\begin{array}{c}17 / 1597 \\
(1.1 \%)\end{array}$ & $5.5-14.3$ & 1 & $\begin{array}{c}104 / 1597 \\
(6.5 \%)\end{array}$ & $65.5-498.9$ & 0.0087 \\
\hline Estradiol & $0 / 1(0 \%)$ & N/A & $0 / 1(0 \%)$ & N/A & N/A & N/A & 1 & N/A & N/A & 1 \\
\hline FSH & $0 / 3(0 \%)$ & N/A & $1 / 3(33.3 \%)$ & $\begin{array}{c}61.09- \\
61.09\end{array}$ & N/A & N/A & 1 & N/A & N/A & 1 \\
\hline LH & $0 / 1(0 \%)$ & N/A & $0 / 1(0 \%)$ & N/A & N/A & N/A & 1 & N/A & N/A & 1 \\
\hline Progesterone & $0 / 1(0 \%)$ & N/A & $0 / 1(0 \%)$ & N/A & N/A & N/A & 1 & N/A & N/A & 1 \\
\hline $\begin{array}{c}\text { Total } \\
\text { Testosterone }\end{array}$ & $\begin{array}{c}1460 / 7873 \\
(18.5 \%)\end{array}$ & $2.5-134.9$ & $\begin{array}{c}729 / 7873 \\
(9.3 \%)\end{array}$ & $\begin{array}{c}48.18- \\
1010\end{array}$ & $\begin{array}{c}528 / 1830 \\
(28.9 \%)\end{array}$ & $2.56-159.2$ & 0 & $\begin{array}{c}130 / 1830 \\
(7.1 \%)\end{array}$ & $48.2-624.3$ & 0.004 \\
\hline SHBG & $\begin{array}{c}215 / 7657 \\
(2.8 \%)\end{array}$ & $6.5-24.4$ & $\begin{array}{c}1949 / 7657 \\
(25.5 \%)\end{array}$ & $\begin{array}{c}122.1- \\
844.9\end{array}$ & $\begin{array}{c}79 / 1798 \\
(4.4 \%)\end{array}$ & $5.14-24.5$ & 0.0006 & $\begin{array}{c}470 / 1798 \\
(26.1 \%)\end{array}$ & $\begin{array}{c}122.1- \\
562.2\end{array}$ & 0.5686 \\
\hline DHEA-S & $\begin{array}{c}649 / 7673 \\
(8.5 \%)\end{array}$ & $0.1-159.3$ & $\begin{array}{c}586 / 7673 \\
(7.6 \%)\end{array}$ & $\begin{array}{c}256.4- \\
881.1\end{array}$ & $\begin{array}{c}152 / 1779 \\
(8.5 \%)\end{array}$ & $5.9-147.9$ & 0.9442 & $\begin{array}{c}127 / 1779 \\
(7.1 \%)\end{array}$ & $\begin{array}{c}261.6- \\
762.4\end{array}$ & 0.5046 \\
\hline IGF-I & $\begin{array}{c}101 / 2826 \\
(3.6 \%)\end{array}$ & $16.78-203$ & $\begin{array}{c}151 / 2826 \\
(5.3 \%)\end{array}$ & $198-572$ & $\begin{array}{l}39 / 845 \\
(4.6 \%)\end{array}$ & $21.06-106$ & 0.199 & $\begin{array}{l}61 / 845 \\
(7.2 \%)\end{array}$ & $221,585.18$ & 0.0492 \\
\hline Estriol & 0/1335 (0\%) & N/A & $\begin{array}{c}27 / 1335 \\
(2 \%)\end{array}$ & $\begin{array}{c}300.7- \\
880.9\end{array}$ & $0 / 163(0 \%)$ & N/A & 1 & $\begin{array}{l}7 / 163 \\
(4.3 \%)\end{array}$ & $\begin{array}{c}305.8- \\
677.4\end{array}$ & 0.1187 \\
\hline Estrone & $\begin{array}{c}287 / 1907 \\
(15 \%)\end{array}$ & $9-16.5$ & $\begin{array}{c}36 / 1907 \\
(1.9 \%)\end{array}$ & $\begin{array}{l}189.6- \\
859.52\end{array}$ & $\begin{array}{c}26 / 211 \\
(12.3 \%)\end{array}$ & $9.3-16.44$ & 0.3385 & $\begin{array}{l}9 / 211 \\
(4.3 \%)\end{array}$ & $\begin{array}{c}186.4- \\
374.4\end{array}$ & 0.0433 \\
\hline Prolactin & $3 / 152(2 \%)$ & $3.92-4.78$ & $\begin{array}{c}28 / 152 \\
(18.4 \%)\end{array}$ & $\begin{array}{c}23.51- \\
112.9\end{array}$ & $1 / 18(5.6 \%)$ & $4.62-4.62$ & 0.3636 & $\begin{array}{c}4 / 18 \\
(22.2 \%)\end{array}$ & $\begin{array}{c}25.46- \\
52.03\end{array}$ & 0.7501 \\
\hline
\end{tabular}


Table S4. Age group 15 - 49. Reproductive hormonal changes in women with positive anti-Tg. The significance was not considered when the total number of subjects tested for each hormonal group is $\leq 10$.

\begin{tabular}{|c|c|c|c|c|c|c|c|c|c|c|}
\hline & \multicolumn{4}{|c|}{ Anti-Tg+ $(\mathrm{n}=7019)$} & \multicolumn{6}{|c|}{ Anti-Tg- $(\mathrm{n}=3524)$} \\
\hline & Reduced & $\begin{array}{c}\text { Range } \\
\text { (reduced) }\end{array}$ & Elevated & $\begin{array}{c}\text { Range } \\
\text { (elevated) }\end{array}$ & Reduced & $\begin{array}{c}\text { Range } \\
\text { (reduced) }\end{array}$ & $\begin{array}{c}\text { P value } \\
\text { (reduced) }\end{array}$ & Elevated & $\begin{array}{c}\text { Range } \\
\text { (elevated) }\end{array}$ & $\begin{array}{l}\text { P value } \\
\text { (elevated) }\end{array}$ \\
\hline Cortisol & $\begin{array}{c}773 / 6235 \\
(12.4 \%)\end{array}$ & $0.365-6.14$ & $\begin{array}{c}760 / 6235 \\
(12.2 \%)\end{array}$ & $12.1-53.53$ & $\begin{array}{c}398 / 3175 \\
(12.5 \%)\end{array}$ & $0.681-6.13$ & 0.8742 & $\begin{array}{c}384 / 3175 \\
(12.1 \%)\end{array}$ & $12.1-55.37$ & 0.9206 \\
\hline $\begin{array}{c}\text { Parathyroid } \\
\text { Hormone }\end{array}$ & $\begin{array}{c}68 / 5331 \\
(1.3 \%)\end{array}$ & $5.5-14.48$ & $\begin{array}{c}276 / 5331 \\
(5.2 \%)\end{array}$ & $65.5-268.6$ & $\begin{array}{c}17 / 2702 \\
(0.6 \%)\end{array}$ & $8.9-14.37$ & 0.0105 & $\begin{array}{c}140 / 2702 \\
(5.2 \%)\end{array}$ & $\begin{array}{c}65.59- \\
498.9\end{array}$ & 1 \\
\hline Estradiol & $0 / 1(0 \%)$ & N/A & $0 / 1(0 \%)$ & N/A & N/A & N/A & 1 & N/A & N/A & 1 \\
\hline FSH & $0 / 2(0 \%)$ & N/A & $1 / 2(50 \%)$ & $\begin{array}{c}61.09- \\
61.09\end{array}$ & $0 / 1(0 \%)$ & N/A & 1 & $0 / 1(0 \%)$ & N/A & 1 \\
\hline LH & $0 / 1(0 \%)$ & N/A & $0 / 1(0 \%)$ & N/A & N/A & N/A & 1 & N/A & N/A & 1 \\
\hline Progesterone & $0 / 1(0 \%)$ & N/A & $0 / 1(0 \%)$ & N/A & N/A & N/A & 1 & N/A & N/A & 1 \\
\hline $\begin{array}{c}\text { Total } \\
\text { Testosterone }\end{array}$ & $\begin{array}{c}1303 / 6471 \\
(20.1 \%)\end{array}$ & $2.5-159.2$ & $\begin{array}{c}562 / 6471 \\
(8.7 \%)\end{array}$ & $\begin{array}{c}48.18 \text { - } \\
912.2\end{array}$ & $\begin{array}{c}684 / 3231 \\
(21.2 \%)\end{array}$ & $2.5-153.9$ & 0.245 & $\begin{array}{c}296 / 3231 \\
(9.2 \%)\end{array}$ & $48.2-1010$ & 0.4588 \\
\hline SHBG & $\begin{array}{c}189 / 6314 \\
(3 \%)\end{array}$ & $6.5-24.29$ & $\begin{array}{c}1604 / 6314 \\
(25.4 \%)\end{array}$ & $\begin{array}{c}122.1- \\
844.9\end{array}$ & $\begin{array}{c}104 / 3140 \\
(3.3 \%)\end{array}$ & $5.14-24.5$ & 0.4358 & $\begin{array}{c}815 / 3140 \\
(26 \%)\end{array}$ & $122.1-585$ & 0.5797 \\
\hline DHEA-S & $\begin{array}{c}525 / 6299 \\
(8.3 \%)\end{array}$ & $0.1-159.3$ & $\begin{array}{c}494 / 6299 \\
(7.8 \%)\end{array}$ & $\begin{array}{c}256.4- \\
881.1\end{array}$ & $\begin{array}{c}276 / 3153 \\
(8.8 \%)\end{array}$ & $\begin{array}{c}0.109- \\
147.9\end{array}$ & 0.5155 & $\begin{array}{c}218 / 3153 \\
(6.9 \%)\end{array}$ & $\begin{array}{c}258.4- \\
703.4\end{array}$ & 0.1161 \\
\hline IGF-I & $\begin{array}{c}90 / 2461 \\
(3.7 \%)\end{array}$ & $21.06-203$ & $\begin{array}{c}142 / 2461 \\
(5.8 \%)\end{array}$ & 198,572 & $\begin{array}{c}50 / 1209 \\
(4.1 \%)\end{array}$ & $16.78-106$ & 0.5354 & $\begin{array}{c}69 / 1209 \\
(5.7 \%)\end{array}$ & $198,585.18$ & 0.9989 \\
\hline Estriol & $0 / 962(0 \%)$ & N/A & $\begin{array}{l}20 / 962 \\
(2.1 \%)\end{array}$ & $\begin{array}{c}300.7- \\
851.8\end{array}$ & $0 / 537(0 \%)$ & N/A & 1 & $\begin{array}{l}14 / 537 \\
(2.6 \%)\end{array}$ & $\begin{array}{c}305.8- \\
880.9\end{array}$ & 0.633 \\
\hline Estrone & $\begin{array}{c}188 / 1381 \\
(13.6 \%)\end{array}$ & $9.1-16.2$ & $\begin{array}{c}25 / 1381 \\
(1.8 \%)\end{array}$ & $\begin{array}{c}188.95- \\
859.52\end{array}$ & $\begin{array}{l}126 / 739 \\
(17.1 \%)\end{array}$ & $9-16.5$ & 0.0395 & $\begin{array}{l}20 / 739 \\
(2.7 \%)\end{array}$ & $\begin{array}{l}186.4- \\
625.31\end{array}$ & 0.2279 \\
\hline Prolactin & $2 / 101(2 \%)$ & $4.78-4.78$ & $\begin{array}{c}20 / 101 \\
(19.8 \%)\end{array}$ & $\begin{array}{c}23.51- \\
112.9\end{array}$ & $2 / 69(2.9 \%)$ & $3.92-4.62$ & 1 & $\begin{array}{c}12 / 69 \\
(17.4 \%)\end{array}$ & $\begin{array}{c}23.67- \\
52.03\end{array}$ & 0.8453 \\
\hline
\end{tabular}

Table S5. Age group 15 - 25. Reproductive hormonal changes in women with hypothyroidism. The significance was not considered when the total number of subjects tested for each hormonal group is $\leq 10$.

\begin{tabular}{|c|c|c|c|c|c|c|c|c|c|c|}
\hline & \multicolumn{4}{|c|}{ Hypothyroidism ( $\mathrm{n}=97)$} & \multicolumn{6}{|c|}{ Thyroid Negative $(\mathrm{n}=1264)$} \\
\hline & Reduced & $\begin{array}{c}\text { Range } \\
\text { (reduced) }\end{array}$ & Elevated & $\begin{array}{c}\text { Range } \\
\text { (elevated) }\end{array}$ & Reduced & $\begin{array}{c}\text { Range } \\
\text { (reduced) }\end{array}$ & $\begin{array}{c}\text { P value } \\
\text { (reduced) }\end{array}$ & Elevated & $\begin{array}{c}\text { Range } \\
\text { (elevated) }\end{array}$ & $\begin{array}{c}\text { P value } \\
\text { (elevated) }\end{array}$ \\
\hline Cortisol & $\begin{array}{c}5 / 88 \\
(5.7 \%)\end{array}$ & $0.558-5.95$ & $\begin{array}{c}33 / 88 \\
(37.5 \%)\end{array}$ & $19.46-46$ & $\begin{array}{c}156 / 1167 \\
(13.4 \%)\end{array}$ & $0.81-6.1$ & 0.0556 & $\begin{array}{c}245 / 1167 \\
(21 \%)\end{array}$ & $\begin{array}{c}12.59- \\
50.64\end{array}$ & 0.0005 \\
\hline $\begin{array}{l}\text { Parathyroid } \\
\text { Hormone }\end{array}$ & $\begin{array}{c}1 / 77 \\
(1.3 \%)\end{array}$ & $\begin{array}{c}13.74- \\
13.74\end{array}$ & $\begin{array}{c}1 / 77 \\
(1.3 \%)\end{array}$ & $\begin{array}{c}66.48- \\
66.48\end{array}$ & $\begin{array}{c}23 / 1009 \\
(2.3 \%)\end{array}$ & $5.5-14.48$ & 1 & $\begin{array}{c}10 / 1009 \\
(1 \%)\end{array}$ & $\begin{array}{c}66.24- \\
84.55\end{array}$ & 0.5564 \\
\hline Estradiol & N/A & N/A & N/A & N/A & N/A & N/A & N/A & N/A & N/A & N/A \\
\hline FSH & N/A & N/A & N/A & N/A & N/A & N/A & N/A & N/A & N/A & N/A \\
\hline LH & N/A & N/A & N/A & N/A & N/A & N/A & N/A & N/A & N/A & N/A \\
\hline Progesterone & N/A & N/A & N/A & N/A & N/A & N/A & N/A & N/A & N/A & N/A \\
\hline
\end{tabular}




\section{Continued}

\begin{tabular}{|c|c|c|c|c|c|c|c|c|c|c|}
\hline $\begin{array}{c}\text { Total } \\
\text { Testosterone }\end{array}$ & $\begin{array}{l}7 / 87 \\
(8 \%)\end{array}$ & $4.55-42.8$ & $\begin{array}{c}11 / 87 \\
(12.6 \%)\end{array}$ & $\begin{array}{c}48.84- \\
80.34\end{array}$ & $\begin{array}{c}131 / 1145 \\
(11.4 \%)\end{array}$ & $2.53-72.9$ & 0.4286 & $\begin{array}{c}146 / 1145 \\
(12.8 \%)\end{array}$ & $48.3-537.1$ & 1 \\
\hline SHBG & $\begin{array}{c}6 / 85 \\
(7.1 \%)\end{array}$ & $6.97-23.62$ & $\begin{array}{c}23 / 85 \\
(27.1 \%)\end{array}$ & $\begin{array}{c}123.3- \\
555.3\end{array}$ & $\begin{array}{c}55 / 1125 \\
(4.9 \%)\end{array}$ & $5.14-24.1$ & 0.5322 & $\begin{array}{c}274 / 1125 \\
(24.4 \%)\end{array}$ & $\begin{array}{c}122.1- \\
562.1\end{array}$ & 0.6689 \\
\hline DHEA-S & $\begin{array}{c}9 / 89 \\
(10.1 \%)\end{array}$ & $\begin{array}{c}55.12- \\
145.9\end{array}$ & $\begin{array}{c}7 / 89 \\
(7.9 \%)\end{array}$ & $\begin{array}{c}285.5- \\
574.8\end{array}$ & $\begin{array}{c}118 / 1134 \\
(10.4 \%)\end{array}$ & $\begin{array}{c}31.19- \\
147.9\end{array}$ & 1 & $\begin{array}{c}143 / 1134 \\
(12.6 \%)\end{array}$ & $300.7-761$ & 0.2517 \\
\hline IGF-I & $\begin{array}{c}1 / 27 \\
(3.7 \%)\end{array}$ & $141-141$ & $\begin{array}{c}4 / 27 \\
(14.8 \%)\end{array}$ & $267-413.83$ & $\begin{array}{l}14 / 428 \\
(3.3 \%)\end{array}$ & $59.1-203$ & 0.6064 & $\begin{array}{c}71 / 428 \\
(16.6 \%)\end{array}$ & $306.08-572$ & 1 \\
\hline Estriol & $\begin{array}{l}0 / 24 \\
(0 \%)\end{array}$ & N/A & $0 / 24(0 \%)$ & N/A & $0 / 227(0 \%)$ & N/A & N/A & $2 / 227(0.9 \%)$ & $351.3-509$ & 1 \\
\hline Estrone & $\begin{array}{c}6 / 24 \\
(25 \%)\end{array}$ & $12.5-14.8$ & $0 / 24(0 \%)$ & N/A & $\begin{array}{l}59 / 274 \\
(21.5 \%)\end{array}$ & $9.3-15.9$ & 0.8913 & $3 / 274(1.1 \%)$ & $\begin{array}{c}186.4- \\
345.4\end{array}$ & 1 \\
\hline Prolactin & $0 / 7(0 \%)$ & N/A & $\begin{array}{c}2 / 7 \\
(28.6 \%)\end{array}$ & $\begin{array}{c}33.46- \\
34.02\end{array}$ & $0 / 22(0 \%)$ & N/A & N/A & $4 / 22(18.2 \%)$ & $\begin{array}{c}26.54- \\
75.58\end{array}$ & 0.6119 \\
\hline
\end{tabular}

Table S6. Age group 15 - 25. Reproductive hormonal changes in women with hyperthyroidism. The significance was not considered when the total number of subjects tested for each hormonal group is $\leq 10$.

\begin{tabular}{|c|c|c|c|c|c|c|c|c|c|c|}
\hline & \multicolumn{4}{|c|}{ Hyperthyroidism $(\mathrm{n}=17)$} & \multicolumn{6}{|c|}{ Thyroid Negative $(\mathrm{n}=1264)$} \\
\hline & Reduced & $\begin{array}{c}\text { Range } \\
\text { (reduced) }\end{array}$ & Elevated & $\begin{array}{c}\text { Range } \\
\text { (elevated) }\end{array}$ & Reduced & $\begin{array}{c}\text { Range } \\
\text { (reduced) }\end{array}$ & $\begin{array}{l}\text { P value } \\
\text { (reduced) }\end{array}$ & Elevated & $\begin{array}{c}\text { Range } \\
\text { (elevated) }\end{array}$ & $\begin{array}{l}\text { P value } \\
\text { (elevated) }\end{array}$ \\
\hline Cortisol & $\begin{array}{c}3 / 17 \\
(17.6 \%)\end{array}$ & $4.12-5.8$ & $\begin{array}{c}2 / 17 \\
(11.8 \%)\end{array}$ & $19.94-20.2$ & $\begin{array}{c}156 / 1167 \\
(13.4 \%)\end{array}$ & $0.81-6.1$ & 0.4896 & $\begin{array}{c}245 / 1167 \\
(21 \%)\end{array}$ & $\begin{array}{c}12.59- \\
50.64\end{array}$ & 0.5485 \\
\hline $\begin{array}{c}\text { Parathyroid } \\
\text { Hormone }\end{array}$ & $\begin{array}{l}0 / 12 \\
(0 \%)\end{array}$ & N/A & $\begin{array}{l}0 / 12 \\
(0 \%)\end{array}$ & N/A & $\begin{array}{c}23 / 1009 \\
(2.3 \%)\end{array}$ & $5.5-14.48$ & 1 & $\begin{array}{c}10 / 1009 \\
(1 \%)\end{array}$ & $\begin{array}{c}66.24- \\
84.55\end{array}$ & 1 \\
\hline Estradiol & N/A & N/A & N/A & N/A & N/A & N/A & N/A & N/A & N/A & N/A \\
\hline FSH & N/A & N/A & N/A & N/A & N/A & N/A & N/A & N/A & N/A & N/A \\
\hline LH & N/A & N/A & N/A & N/A & N/A & N/A & N/A & N/A & N/A & N/A \\
\hline Progesterone & N/A & N/A & N/A & N/A & N/A & N/A & N/A & N/A & N/A & N/A \\
\hline $\begin{array}{c}\text { Total } \\
\text { Testosterone }\end{array}$ & $\begin{array}{c}1 / 14 \\
(7.1 \%)\end{array}$ & $34.9-34.9$ & $\begin{array}{c}1 / 14 \\
(7.1 \%)\end{array}$ & $78-78$ & $\begin{array}{c}131 / 1145 \\
(11.4 \%)\end{array}$ & $2.53-72.9$ & 1 & $\begin{array}{c}146 / 1145 \\
(12.8 \%)\end{array}$ & $48.3-537.1$ & 1 \\
\hline SHBG & $\begin{array}{l}0 / 15 \\
(0 \%)\end{array}$ & N/A & $\begin{array}{c}6 / 15 \\
(40 \%)\end{array}$ & $\begin{array}{c}122.2- \\
177.7\end{array}$ & $\begin{array}{c}55 / 1125 \\
(4.9 \%)\end{array}$ & $5.14-24.1$ & 1 & $\begin{array}{c}274 / 1125 \\
(24.4 \%)\end{array}$ & $\begin{array}{c}122.1- \\
562.1\end{array}$ & 0.2729 \\
\hline DHEA-S & $\begin{array}{c}2 / 15 \\
(13.3 \%)\end{array}$ & $\begin{array}{c}109.4- \\
135.5\end{array}$ & $\begin{array}{c}2 / 15 \\
(13.3 \%)\end{array}$ & $\begin{array}{c}340.9- \\
416.4\end{array}$ & $\begin{array}{c}118 / 1134 \\
(10.4 \%)\end{array}$ & $\begin{array}{c}31.19- \\
147.9\end{array}$ & 0.6646 & $\begin{array}{c}143 / 1134 \\
(12.6 \%)\end{array}$ & $300.7-761$ & 1 \\
\hline IGF-I & $0 / 9(0 \%)$ & N/A & $\begin{array}{c}4 / 9 \\
(44.4 \%)\end{array}$ & $\begin{array}{c}421.511 \text { - } \\
554.8\end{array}$ & $\begin{array}{l}14 / 428 \\
(3.3 \%)\end{array}$ & $59.1-203$ & 1 & $\begin{array}{c}71 / 428 \\
(16.6 \%)\end{array}$ & $306.08-572$ & 0.0509 \\
\hline Estriol & $0 / 1(0 \%)$ & N/A & $0 / 1(0 \%)$ & N/A & $0 / 227(0 \%)$ & N/A & 1 & $2 / 227(0.9 \%)$ & $351.3-509$ & 1 \\
\hline Estrone & $\begin{array}{c}1 / 1 \\
(100 \%)\end{array}$ & $16.2-16.2$ & $0 / 1(0 \%)$ & N/A & $\begin{array}{c}59 / 274 \\
(21.5 \%)\end{array}$ & $9.3-15.9$ & 0.2182 & $3 / 274(1.1 \%)$ & $\begin{array}{c}186.4- \\
345.4\end{array}$ & 1 \\
\hline Prolactin & N/A & N/A & N/A & N/A & $0 / 22(0 \%)$ & N/A & 1 & $4 / 22(18.2 \%)$ & $\begin{array}{c}26.54- \\
75.58\end{array}$ & 1 \\
\hline
\end{tabular}


Table S7. Age group 15 - 25. Reproductive hormonal changes in women with positive anti-TPO. The significance was not considered when the total number of subjects tested for each hormonal group is $\leq 10$.

\begin{tabular}{|c|c|c|c|c|c|c|c|c|c|c|}
\hline & \multicolumn{4}{|c|}{ Anti-TPO+ $(\mathrm{n}=1166)$} & \multicolumn{6}{|c|}{ Anti-TPO- $(\mathrm{n}=253)$} \\
\hline & Reduced & $\begin{array}{c}\text { Range } \\
\text { (reduced) }\end{array}$ & Elevated & $\begin{array}{c}\text { Range } \\
\text { (elevated) }\end{array}$ & Reduced & $\begin{array}{c}\text { Range } \\
\text { (reduced) }\end{array}$ & $\begin{array}{l}\text { P value } \\
\text { (reduced) }\end{array}$ & Elevated & $\begin{array}{c}\text { Range } \\
\text { (elevated) }\end{array}$ & $\begin{array}{c}\text { P value } \\
\text { (elevated) }\end{array}$ \\
\hline Cortisol & $\begin{array}{c}142 / 1058 \\
(13.4 \%)\end{array}$ & $0.558-6.1$ & $\begin{array}{c}242 / 1058 \\
(22.9 \%)\end{array}$ & $\begin{array}{c}12.59- \\
50.64\end{array}$ & $\begin{array}{c}28 / 243 \\
(11.5 \%)\end{array}$ & $1.36-6.1$ & 0.4924 & $\begin{array}{c}43 / 243 \\
(17.7 \%)\end{array}$ & $15.5-45.5$ & 0.0942 \\
\hline $\begin{array}{c}\text { Parathyroid } \\
\text { Hormone }\end{array}$ & $\begin{array}{l}17 / 908 \\
(1.9 \%)\end{array}$ & $7.8-14.48$ & $\begin{array}{l}13 / 908 \\
(1.4 \%)\end{array}$ & $\begin{array}{c}66.24- \\
268.6\end{array}$ & $\begin{array}{l}7 / 218 \\
(3.2 \%)\end{array}$ & $5.5-13.8$ & 0.3331 & $0 / 218(0 \%)$ & N/A & 0.0851 \\
\hline Estradiol & N/A & N/A & N/A & N/A & N/A & N/A & N/A & N/A & N/A & N/A \\
\hline FSH & N/A & N/A & N/A & N/A & N/A & N/A & N/A & N/A & N/A & N/A \\
\hline LH & N/A & N/A & N/A & N/A & N/A & N/A & N/A & N/A & N/A & N/A \\
\hline Progesterone & N/A & N/A & N/A & N/A & N/A & N/A & N/A & N/A & N/A & N/A \\
\hline $\begin{array}{c}\text { Total } \\
\text { Testosterone }\end{array}$ & $\begin{array}{c}88 / 1043 \\
(8.4 \%)\end{array}$ & $2.53-72.9$ & $\begin{array}{c}143 / 1043 \\
(13.7 \%)\end{array}$ & $48.3-537.1$ & $\begin{array}{c}54 / 238 \\
(22.7 \%)\end{array}$ & $2.88-60.1$ & 0 & $\begin{array}{l}20 / 238 \\
(8.4 \%)\end{array}$ & $49.39-79.6$ & 0.0349 \\
\hline SHBG & $\begin{array}{c}42 / 1024 \\
(4.1 \%)\end{array}$ & $6.97-23.62$ & $\begin{array}{c}261 / 1024 \\
(25.5 \%)\end{array}$ & $\begin{array}{c}122.1- \\
562.1\end{array}$ & $\begin{array}{l}22 / 235 \\
(9.4 \%)\end{array}$ & $5.14-24.1$ & 0.0017 & $\begin{array}{c}45 / 235 \\
(19.1 \%)\end{array}$ & $\begin{array}{c}122.9- \\
350.8\end{array}$ & 0.0501 \\
\hline DHEA-S & $\begin{array}{c}113 / 1040 \\
(10.9 \%)\end{array}$ & $31.19-146$ & $\begin{array}{c}122 / 1040 \\
(11.7 \%)\end{array}$ & $\begin{array}{c}322.7- \\
658.4\end{array}$ & $\begin{array}{l}20 / 237 \\
(8.4 \%)\end{array}$ & $62.4-147.9$ & 0.3242 & $\begin{array}{c}33 / 237 \\
(13.9 \%)\end{array}$ & $285.5-761$ & 0.4106 \\
\hline IGF-I & $\begin{array}{l}15 / 359 \\
(4.2 \%)\end{array}$ & $59.1-203$ & $\begin{array}{c}48 / 359 \\
(13.4 \%)\end{array}$ & $267-572$ & $\begin{array}{l}1 / 117 \\
(0.9 \%)\end{array}$ & $106-106$ & 0.135 & $\begin{array}{c}33 / 117 \\
(28.2 \%)\end{array}$ & $\begin{array}{c}312.01- \\
536.42\end{array}$ & 0.0004 \\
\hline Estriol & $0 / 237(0 \%)$ & N/A & $\begin{array}{l}2 / 237 \\
(0.8 \%)\end{array}$ & $351.3-509$ & $0 / 23(0 \%)$ & N/A & N/A & $0 / 23(0 \%)$ & N/A & N/A \\
\hline Estrone & $\begin{array}{c}65 / 280 \\
(23.2 \%)\end{array}$ & $9.3-16.2$ & $\begin{array}{l}2 / 280 \\
(0.7 \%)\end{array}$ & $\begin{array}{c}345.4- \\
345.4\end{array}$ & $\begin{array}{c}5 / 29 \\
(17.2 \%)\end{array}$ & $12.1-13.6$ & 0.6182 & $\begin{array}{c}1 / 29 \\
(3.4 \%)\end{array}$ & $\begin{array}{c}186.4- \\
186.4\end{array}$ & 0.2567 \\
\hline Prolactin & $0 / 28(0 \%)$ & N/A & $\begin{array}{c}5 / 28 \\
(17.9 \%)\end{array}$ & $\begin{array}{c}26.54- \\
112.9\end{array}$ & $0 / 3(0 \%)$ & N/A & N/A & $\begin{array}{c}2 / 3 \\
(66.7 \%)\end{array}$ & $\begin{array}{c}33.46- \\
52.03\end{array}$ & 0.1199 \\
\hline
\end{tabular}

Table S8. Age group 15-25. Reproductive hormonal changes in women with positive anti-Tg. The significance was not considered when the total number of subjects tested for each hormonal group is $\leq 10$.

\begin{tabular}{|c|c|c|c|c|c|c|c|c|c|c|}
\hline & \multicolumn{4}{|c|}{ Anti-Tg+ $(\mathrm{n}=898)$} & \multicolumn{6}{|c|}{ Anti-Tg- $(\mathrm{n}=520)$} \\
\hline & Reduced & $\begin{array}{c}\text { Range } \\
\text { (reduced) }\end{array}$ & Elevated & $\begin{array}{c}\text { Range } \\
\text { (elevated) }\end{array}$ & Reduced & $\begin{array}{c}\text { Range } \\
\text { (reduced) }\end{array}$ & $\begin{array}{l}\text { P value } \\
\text { (reduced) }\end{array}$ & Elevated & $\begin{array}{c}\text { Range } \\
\text { (elevated) }\end{array}$ & $\begin{array}{c}\text { P value } \\
\text { (elevated) }\end{array}$ \\
\hline Cortisol & $\begin{array}{c}99 / 823 \\
(12 \%)\end{array}$ & $0.558-6.1$ & $\begin{array}{l}185 / 823 \\
(22.5 \%)\end{array}$ & $12.7-46.82$ & $\begin{array}{l}71 / 477 \\
(14.9 \%)\end{array}$ & $1.77-6.1$ & 0.1656 & $\begin{array}{c}100 / 477 \\
(21 \%)\end{array}$ & $\begin{array}{c}12.59- \\
50.64\end{array}$ & 0.571 \\
\hline $\begin{array}{l}\text { Parathyroid } \\
\text { Hormone }\end{array}$ & $\begin{array}{l}18 / 700 \\
(2.6 \%)\end{array}$ & $5.5-14.48$ & $\begin{array}{l}9 / 700 \\
(1.3 \%)\end{array}$ & $\begin{array}{c}66.24- \\
268.6\end{array}$ & $\begin{array}{c}6 / 425 \\
(1.4 \%)\end{array}$ & $10-12.43$ & 0.2747 & $\begin{array}{l}4 / 425 \\
(0.9 \%)\end{array}$ & $\begin{array}{c}66.48- \\
75.95\end{array}$ & 0.7762 \\
\hline Estradiol & N/A & N/A & N/A & N/A & N/A & N/A & N/A & $\mathrm{N} / \mathrm{A}$ & N/A & N/A \\
\hline FSH & N/A & N/A & N/A & N/A & N/A & N/A & N/A & N/A & N/A & N/A \\
\hline LH & N/A & N/A & N/A & N/A & N/A & N/A & N/A & N/A & N/A & N/A \\
\hline Progesterone & N/A & N/A & N/A & N/A & N/A & N/A & N/A & N/A & N/A & N/A \\
\hline
\end{tabular}




\section{Continued}

\begin{tabular}{|c|c|c|c|c|c|c|c|c|c|c|}
\hline $\begin{array}{c}\text { Total } \\
\text { Testosterone }\end{array}$ & $\begin{array}{c}88 / 805 \\
(10.9 \%)\end{array}$ & $2.65-60.1$ & $\begin{array}{c}105 / 805 \\
(13 \%)\end{array}$ & $48.3-537.1$ & $\begin{array}{c}54 / 475 \\
(11.4 \%)\end{array}$ & $2.53-72.9$ & 0.8821 & $\begin{array}{l}57 / 475 \\
(12 \%)\end{array}$ & $48.4-103$ & 0.6488 \\
\hline SHBG & $\begin{array}{l}38 / 797 \\
(4.8 \%)\end{array}$ & $6.97-24.1$ & $\begin{array}{l}195 / 797 \\
(24.5 \%)\end{array}$ & $122.1-555.3$ & $\begin{array}{l}25 / 461 \\
(5.4 \%)\end{array}$ & $5.14-23.3$ & 0.7046 & $\begin{array}{l}111 / 461 \\
(24.1 \%)\end{array}$ & $\begin{array}{c}123.9- \\
562.1\end{array}$ & 0.931 \\
\hline DHEA-S & $\begin{array}{l}77 / 800 \\
(9.6 \%)\end{array}$ & $37.49-146$ & $\begin{array}{c}97 / 800 \\
(12.1 \%)\end{array}$ & $285.5-645.4$ & $\begin{array}{c}56 / 476 \\
(11.8 \%)\end{array}$ & $\begin{array}{c}31.19- \\
147.9\end{array}$ & 0.2649 & $\begin{array}{l}57 / 476 \\
(12 \%)\end{array}$ & $\begin{array}{c}301.6- \\
658.4\end{array}$ & 1 \\
\hline IGF-I & $\begin{array}{l}11 / 320 \\
(3.4 \%)\end{array}$ & $75.4-203$ & $\begin{array}{c}51 / 320 \\
(15.9 \%)\end{array}$ & $306.08-572$ & $\begin{array}{l}5 / 155 \\
(3.2 \%)\end{array}$ & $59.1-106$ & 1 & $\begin{array}{l}29 / 155 \\
(18.7 \%)\end{array}$ & $\begin{array}{c}267- \\
536.42\end{array}$ & 0.5312 \\
\hline Estriol & $\begin{array}{c}0 / 169 \\
(0 \%)\end{array}$ & N/A & $\begin{array}{l}1 / 169 \\
(0.6 \%)\end{array}$ & $351.3-351.3$ & $0 / 91(0 \%)$ & N/A & N/A & $\begin{array}{c}1 / 91 \\
(1.1 \%)\end{array}$ & $509-509$ & 1 \\
\hline Estrone & $\begin{array}{c}47 / 203 \\
(23.2 \%)\end{array}$ & $9.3-16.2$ & $0 / 203(0 \%)$ & N/A & $\begin{array}{c}23 / 106 \\
(21.7 \%)\end{array}$ & $9.7-15.9$ & 0.8833 & $\begin{array}{l}3 / 106 \\
(2.8 \%)\end{array}$ & $\begin{array}{c}186.4- \\
345.4\end{array}$ & 0.0396 \\
\hline Prolactin & $0 / 17(0 \%)$ & N/A & $\begin{array}{c}5 / 17 \\
(29.4 \%)\end{array}$ & $26.54-112.9$ & N/A & N/A & N/A & $\begin{array}{c}2 / 14 \\
(14.3 \%)\end{array}$ & $\begin{array}{c}29.68- \\
52.03\end{array}$ & 0.4117 \\
\hline
\end{tabular}

Table S9. Age group 26 - 35. Reproductive hormonal changes in women with hypothyroidism. The significance was not considered when the total number of subjects tested for each hormonal group is $\leq 10$.

\begin{tabular}{|c|c|c|c|c|c|c|c|c|c|c|}
\hline & \multicolumn{4}{|c|}{ Hypothyroidism $(\mathrm{n}=157)$} & \multicolumn{6}{|c|}{ Thyroid Negative $(\mathrm{n}=2633)$} \\
\hline & Reduced & $\begin{array}{c}\text { Range } \\
\text { (reduced) }\end{array}$ & Elevated & $\begin{array}{c}\text { Range } \\
\text { (elevated) }\end{array}$ & Reduced & $\begin{array}{l}\text { Range } \\
\text { (reduced) }\end{array}$ & $\begin{array}{l}\text { P value } \\
\text { (reduced) }\end{array}$ & Elevated & $\begin{array}{c}\text { Range } \\
\text { (elevated) }\end{array}$ & $\begin{array}{l}\text { P value } \\
\text { (elevated) }\end{array}$ \\
\hline Cortisol & $\begin{array}{c}20 / 143 \\
(14 \%)\end{array}$ & $1.78-6.12$ & $\begin{array}{c}26 / 143 \\
(18.2 \%)\end{array}$ & $18.5-42.23$ & $\begin{array}{c}243 / 2371 \\
(10.2 \%)\end{array}$ & $0.611-6.13$ & 0.2015 & $\begin{array}{c}377 / 2371 \\
(15.9 \%)\end{array}$ & $\begin{array}{c}12.31- \\
55.37\end{array}$ & 0.5453 \\
\hline $\begin{array}{c}\text { Parathyroid } \\
\text { Hormone }\end{array}$ & $0 / 125(0 \%)$ & N/A & $\begin{array}{c}9 / 125 \\
(7.2 \%)\end{array}$ & $65.6-498.9$ & $\begin{array}{c}27 / 2035 \\
(1.3 \%)\end{array}$ & $7-14.37$ & 0.4007 & $\begin{array}{c}83 / 2035 \\
(4.1 \%)\end{array}$ & $65.6-149.5$ & 0.1473 \\
\hline Estradiol & N/A & N/A & N/A & N/A & N/A & N/A & $\mathrm{N} / \mathrm{A}$ & N/A & N/A & N/A \\
\hline FSH & N/A & N/A & N/A & N/A & N/A & N/A & N/A & N/A & N/A & N/A \\
\hline LH & N/A & N/A & N/A & N/A & N/A & N/A & N/A & N/A & N/A & N/A \\
\hline Progesterone & N/A & N/A & N/A & N/A & N/A & N/A & N/A & N/A & N/A & N/A \\
\hline $\begin{array}{c}\text { Total } \\
\text { Testosterone }\end{array}$ & $\begin{array}{c}17 / 144 \\
(11.8 \%)\end{array}$ & $4.23-51.6$ & $\begin{array}{l}12 / 144 \\
(8.3 \%)\end{array}$ & $\begin{array}{c}48.61- \\
141.9\end{array}$ & $\begin{array}{c}374 / 2414 \\
(15.5 \%)\end{array}$ & $2.52-121.6$ & 0.2822 & $\begin{array}{c}214 / 2414 \\
(8.9 \%)\end{array}$ & $48.2-695.6$ & 0.9464 \\
\hline SHBG & $\begin{array}{c}6 / 141 \\
(4.3 \%)\end{array}$ & $\begin{array}{c}15.81- \\
23.26\end{array}$ & $\begin{array}{c}32 / 141 \\
(22.7 \%)\end{array}$ & $\begin{array}{c}123.1- \\
844.9\end{array}$ & $\begin{array}{c}64 / 2339 \\
(2.7 \%)\end{array}$ & $7.72-24.23$ & 0.4261 & $\begin{array}{c}671 / 2339 \\
(28.7 \%)\end{array}$ & $\begin{array}{c}122.2- \\
732.3\end{array}$ & 0.1507 \\
\hline DHEA-S & $\begin{array}{c}19 / 143 \\
(13.3 \%)\end{array}$ & $\begin{array}{c}10.92- \\
142.7\end{array}$ & $\begin{array}{l}14 / 143 \\
(9.8 \%)\end{array}$ & $\begin{array}{c}343.2- \\
530.8\end{array}$ & $\begin{array}{c}264 / 2354 \\
(11.2 \%)\end{array}$ & $3.52-159.3$ & 0.5333 & $\begin{array}{c}247 / 2354 \\
(10.5 \%)\end{array}$ & $\begin{array}{c}340.2- \\
762.4\end{array}$ & 0.8998 \\
\hline IGF-I & $\begin{array}{c}1 / 40 \\
(2.5 \%)\end{array}$ & $\begin{array}{c}41.15- \\
41.15\end{array}$ & $\begin{array}{c}3 / 40 \\
(7.5 \%)\end{array}$ & $288-377$ & $\begin{array}{l}35 / 861 \\
(4.1 \%)\end{array}$ & $\begin{array}{c}16.78- \\
96.01\end{array}$ & 1 & $\begin{array}{l}47 / 861 \\
(5.5 \%)\end{array}$ & $235-585.18$ & 0.4819 \\
\hline Estriol & $0 / 17(0 \%)$ & N/A & $\begin{array}{c}1 / 17 \\
(5.9 \%)\end{array}$ & $\begin{array}{c}635.3- \\
635.3\end{array}$ & $0 / 387(0 \%)$ & N/A & 1 & $\begin{array}{l}13 / 387 \\
(3.4 \%)\end{array}$ & $\begin{array}{c}392.6- \\
851.8\end{array}$ & 0.4577 \\
\hline Estrone & $2 / 25(8 \%)$ & $11.4-11.4$ & $0 / 25(0 \%)$ & N/A & $\begin{array}{c}69 / 502 \\
(13.7 \%)\end{array}$ & $9-16.4$ & 0.5575 & $\begin{array}{l}18 / 502 \\
(3.6 \%)\end{array}$ & $\begin{array}{c}191.02- \\
859.52\end{array}$ & 1 \\
\hline Prolactin & N/A & N/A & N/A & N/A & $1 / 39(2.6 \%)$ & $4.78-4.78$ & 1 & $\begin{array}{c}11 / 39 \\
(28.2 \%)\end{array}$ & $\begin{array}{c}23.51- \\
42.19\end{array}$ & 1 \\
\hline
\end{tabular}


Table S10. Age group 26 - 35. Reproductive hormonal changes in women with hyperthyroidism. The significance was not considered when the total number of subjects tested for each hormonal group is $\leq 10$.

\begin{tabular}{|c|c|c|c|c|c|c|c|c|c|c|}
\hline & \multicolumn{4}{|c|}{ Hyperthyroidism $(\mathrm{n}=66)$} & \multicolumn{6}{|c|}{ Thyroid Negative $(n=2633)$} \\
\hline & Reduced & $\begin{array}{c}\text { Range } \\
\text { (reduced) }\end{array}$ & Elevated & $\begin{array}{c}\text { Range } \\
\text { (elevated) }\end{array}$ & Reduced & $\begin{array}{c}\text { Range } \\
\text { (reduced) }\end{array}$ & $\begin{array}{l}\text { P value } \\
\text { (reduced) }\end{array}$ & Elevated & $\begin{array}{c}\text { Range } \\
\text { (elevated) }\end{array}$ & $\begin{array}{l}\text { P value } \\
\text { (elevated) }\end{array}$ \\
\hline Cortisol & $\begin{array}{c}4 / 60 \\
(6.7 \%)\end{array}$ & $0.801-6$ & $\begin{array}{c}7 / 60 \\
(11.7 \%)\end{array}$ & $13.9-30.6$ & $\begin{array}{c}243 / 2371 \\
(10.2 \%)\end{array}$ & $0.611-6.13$ & 0.515 & $\begin{array}{c}377 / 2371 \\
(15.9 \%)\end{array}$ & $\begin{array}{c}12.31- \\
55.37\end{array}$ & 0.4784 \\
\hline $\begin{array}{c}\text { Parathyroid } \\
\text { Hormone }\end{array}$ & $\begin{array}{c}4 / 52 \\
(7.7 \%)\end{array}$ & $13.1-13.8$ & $\begin{array}{c}2 / 52 \\
(3.8 \%)\end{array}$ & $66.14-79.2$ & $\begin{array}{c}27 / 2035 \\
(1.3 \%)\end{array}$ & $7-14.37$ & 0.0066 & $\begin{array}{c}83 / 2035 \\
(4.1 \%)\end{array}$ & $65.6-149.5$ & 1 \\
\hline Estradiol & N/A & N/A & N/A & N/A & N/A & N/A & N/A & N/A & N/A & N/A \\
\hline FSH & N/A & N/A & N/A & N/A & N/A & N/A & N/A & N/A & N/A & N/A \\
\hline $\mathrm{LH}$ & N/A & N/A & N/A & N/A & N/A & N/A & N/A & N/A & N/A & N/A \\
\hline Progesterone & N/A & N/A & N/A & N/A & N/A & N/A & N/A & N/A & N/A & N/A \\
\hline $\begin{array}{c}\text { Total } \\
\text { Testosterone }\end{array}$ & $\begin{array}{c}7 / 60 \\
(11.7 \%)\end{array}$ & $2.62-49.8$ & $\begin{array}{c}8 / 60 \\
(13.3 \%)\end{array}$ & $49-111.7$ & $\begin{array}{c}374 / 2414 \\
(15.5 \%)\end{array}$ & $2.52-121.6$ & 0.5287 & $\begin{array}{c}214 / 2414 \\
(8.9 \%)\end{array}$ & $48.2-695.6$ & 0.3332 \\
\hline SHBG & $\begin{array}{l}0 / 57 \\
(0 \%)\end{array}$ & N/A & $\begin{array}{c}19 / 57 \\
(33.3 \%)\end{array}$ & $124.3-643$ & $\begin{array}{c}64 / 2339 \\
(2.7 \%)\end{array}$ & $7.72-24.23$ & 0.4034 & $\begin{array}{c}671 / 2339 \\
(28.7 \%)\end{array}$ & $\begin{array}{c}122.2- \\
732.3\end{array}$ & 0.537 \\
\hline DHEA-S & $\begin{array}{c}8 / 57 \\
(14 \%)\end{array}$ & $11.9-89.93$ & $\begin{array}{c}7 / 57 \\
(12.3 \%)\end{array}$ & $354.6-460$ & $\begin{array}{c}264 / 2354 \\
(11.2 \%)\end{array}$ & $3.52-159.3$ & 0.6504 & $\begin{array}{c}247 / 2354 \\
(10.5 \%)\end{array}$ & $\begin{array}{c}340.2- \\
762.4\end{array}$ & 0.8289 \\
\hline IGF-I & $\begin{array}{c}2 / 24 \\
(8.3 \%)\end{array}$ & $58.2-92.36$ & $\begin{array}{c}1 / 24 \\
(4.2 \%)\end{array}$ & $\begin{array}{c}355.16- \\
355.16\end{array}$ & $\begin{array}{l}35 / 861 \\
(4.1 \%)\end{array}$ & $\begin{array}{c}16.78- \\
96.01\end{array}$ & 0.2652 & $\begin{array}{l}47 / 861 \\
(5.5 \%)\end{array}$ & $235-585.18$ & 1 \\
\hline Estriol & $0 / 9(0 \%)$ & N/A & $0 / 9(0 \%)$ & N/A & $0 / 387(0 \%)$ & N/A & 1 & $\begin{array}{l}13 / 387 \\
(3.4 \%)\end{array}$ & $\begin{array}{c}392.6- \\
851.8\end{array}$ & 1 \\
\hline Estrone & $\begin{array}{c}2 / 12 \\
(16.7 \%)\end{array}$ & $11.1-11.1$ & $0 / 12(0 \%)$ & N/A & $\begin{array}{c}69 / 502 \\
(13.7 \%)\end{array}$ & $9-16.4$ & 0.6755 & $\begin{array}{l}18 / 502 \\
(3.6 \%)\end{array}$ & $\begin{array}{c}191.02- \\
859.52\end{array}$ & 1 \\
\hline Prolactin & $0 / 1(0 \%)$ & N/A & $0 / 1(0 \%)$ & N/A & $1 / 39(2.6 \%)$ & $4.78-4.78$ & 1 & $\begin{array}{c}11 / 39 \\
(28.2 \%)\end{array}$ & $\begin{array}{c}23.51- \\
42.19\end{array}$ & 1 \\
\hline
\end{tabular}

Table S11. Age group 26 - 35. Reproductive hormonal changes in women with positive anti-TPO. The significance was not considered when the total number of subjects tested for each hormonal group is $\leq 10$.

\begin{tabular}{|c|c|c|c|c|c|c|c|c|c|c|}
\hline & \multicolumn{4}{|c|}{ Anti-TPO+ $(\mathrm{n}=2376)$} & \multicolumn{6}{|c|}{ Anti-TPO- $(\mathrm{n}=552)$} \\
\hline & Reduced & $\begin{array}{c}\text { Range } \\
\text { (reduced) }\end{array}$ & Elevated & $\begin{array}{c}\text { Range } \\
\text { (elevated) }\end{array}$ & Reduced & $\begin{array}{c}\text { Range } \\
\text { (reduced) }\end{array}$ & $\begin{array}{c}\text { P value } \\
\text { (reduced) }\end{array}$ & Elevated & $\begin{array}{c}\text { Range } \\
\text { (elevated) }\end{array}$ & $\begin{array}{l}\text { P value } \\
\text { (elevated) }\end{array}$ \\
\hline Cortisol & $\begin{array}{c}234 / 2130 \\
(11 \%)\end{array}$ & $0.444-6.13$ & $\begin{array}{c}343 / 2130 \\
(16.1 \%)\end{array}$ & $\begin{array}{c}12.31- \\
55.37\end{array}$ & $\begin{array}{l}43 / 502 \\
(8.6 \%)\end{array}$ & $1.1-6.1$ & 0.1314 & $\begin{array}{c}76 / 502 \\
(15.1 \%)\end{array}$ & $13.4-38.38$ & 0.6432 \\
\hline $\begin{array}{c}\text { Parathyroid } \\
\text { Hormone }\end{array}$ & $\begin{array}{c}30 / 1810 \\
(1.7 \%)\end{array}$ & $7-14.37$ & $\begin{array}{c}70 / 1810 \\
(3.9 \%)\end{array}$ & $65.6-149.5$ & $\begin{array}{c}3 / 449 \\
(0.7 \%)\end{array}$ & $7.5-13.7$ & 0.1835 & $\begin{array}{l}26 / 449 \\
(5.8 \%)\end{array}$ & $65.6-498.9$ & 0.0934 \\
\hline Estradiol & N/A & N/A & N/A & N/A & N/A & N/A & N/A & N/A & N/A & N/A \\
\hline FSH & N/A & N/A & N/A & N/A & N/A & N/A & N/A & N/A & N/A & N/A \\
\hline LH & N/A & N/A & N/A & N/A & N/A & N/A & N/A & N/A & N/A & N/A \\
\hline Progesterone & N/A & N/A & N/A & N/A & N/A & N/A & N/A & N/A & N/A & N/A \\
\hline
\end{tabular}


Continued

\begin{tabular}{|c|c|c|c|c|c|c|c|c|c|c|}
\hline $\begin{array}{c}\text { Total } \\
\text { Testosterone }\end{array}$ & $\begin{array}{c}279 / 2164 \\
(12.9 \%)\end{array}$ & $2.52-121.6$ & $\begin{array}{c}203 / 2164 \\
(9.4 \%)\end{array}$ & $\begin{array}{c}48.21- \\
695.6\end{array}$ & $\begin{array}{l}128 / 517 \\
(24.8 \%)\end{array}$ & $3.3-73.7$ & 0 & $\begin{array}{l}38 / 517 \\
(7.4 \%)\end{array}$ & $48.2-596.5$ & 0.1723 \\
\hline SHBG & $\begin{array}{c}45 / 2095 \\
(2.1 \%)\end{array}$ & $7.8-23.8$ & $\begin{array}{c}597 / 2095 \\
(28.5 \%)\end{array}$ & $\begin{array}{c}122.2- \\
844.9\end{array}$ & $\begin{array}{l}29 / 506 \\
(5.7 \%)\end{array}$ & $7.72-24.5$ & 0 & $\begin{array}{l}140 / 506 \\
(27.7 \%)\end{array}$ & $\begin{array}{c}122.3- \\
562.2\end{array}$ & 0.7519 \\
\hline DHEA-S & $\begin{array}{c}238 / 2129 \\
(11.2 \%)\end{array}$ & $3.52-159.3$ & $\begin{array}{c}217 / 2129 \\
(10.2 \%)\end{array}$ & $\begin{array}{c}340.5- \\
702.8\end{array}$ & $\begin{array}{c}63 / 498 \\
(12.7 \%)\end{array}$ & $\begin{array}{c}16.01- \\
141.5\end{array}$ & 0.3953 & $\begin{array}{c}58 / 498 \\
(11.6 \%)\end{array}$ & $\begin{array}{c}340.2- \\
762.4\end{array}$ & 0.3827 \\
\hline IGF-I & $\begin{array}{l}33 / 707 \\
(4.7 \%)\end{array}$ & $\begin{array}{c}16.78- \\
96.01\end{array}$ & $\begin{array}{l}45 / 707 \\
(6.4 \%)\end{array}$ & $\begin{array}{c}235 \text { - } \\
483.824\end{array}$ & $\begin{array}{l}6 / 246 \\
(2.4 \%)\end{array}$ & $40.58-80.5$ & 0.1826 & $\begin{array}{l}13 / 246 \\
(5.3 \%)\end{array}$ & $\begin{array}{c}274- \\
585.18\end{array}$ & 0.6486 \\
\hline Estriol & $0 / 372(0 \%)$ & N/A & $\begin{array}{l}13 / 372 \\
(3.5 \%)\end{array}$ & $\begin{array}{c}392.6- \\
880.9\end{array}$ & $0 / 49(0 \%)$ & N/A & N/A & $\begin{array}{c}2 / 49 \\
(4.1 \%)\end{array}$ & $\begin{array}{c}677.4- \\
677.4\end{array}$ & 0.6898 \\
\hline Estrone & $\begin{array}{c}66 / 490 \\
(13.5 \%)\end{array}$ & $9-16.4$ & $\begin{array}{l}15 / 490 \\
(3.1 \%)\end{array}$ & $\begin{array}{c}191.02- \\
859.52\end{array}$ & $\begin{array}{c}8 / 59 \\
(13.6 \%)\end{array}$ & $11.7-15.9$ & 1 & $\begin{array}{c}3 / 59 \\
(5.1 \%)\end{array}$ & $\begin{array}{c}228.7- \\
228.7\end{array}$ & 0.4282 \\
\hline Prolactin & $1 / 35(2.9 \%)$ & $4.78-4.78$ & 9/35 (25.7\%) & $\begin{array}{c}23.51- \\
42.19\end{array}$ & $0 / 5(0 \%)$ & N/A & N/A & $2 / 5(40 \%)$ & $\begin{array}{c}25.46- \\
26.78\end{array}$ & 0.603 \\
\hline
\end{tabular}

Table S12. Age group 26-35. Reproductive hormonal changes in women with positive anti-Tg. The significance was not considered when the total number of subjects tested for each hormonal group is $\leq 10$.

\begin{tabular}{|c|c|c|c|c|c|c|c|c|c|c|}
\hline & \multicolumn{4}{|c|}{ Anti-Tg+ $(\mathrm{n}=1964)$} & \multicolumn{6}{|c|}{ Anti-Tg- $(\mathrm{n}=964)$} \\
\hline & Reduced & $\begin{array}{c}\text { Range } \\
\text { (reduced) }\end{array}$ & Elevated & $\begin{array}{c}\text { Range } \\
\text { (elevated) }\end{array}$ & Reduced & $\begin{array}{c}\text { Range } \\
\text { (reduced) }\end{array}$ & $\begin{array}{l}\text { P value } \\
\text { (reduced) }\end{array}$ & Elevated & $\begin{array}{c}\text { Range } \\
\text { (elevated) }\end{array}$ & $\begin{array}{l}\text { P value } \\
\text { (elevated) }\end{array}$ \\
\hline Cortisol & $\begin{array}{c}182 / 1757 \\
(10.4 \%)\end{array}$ & $0.444-6.13$ & $\begin{array}{c}279 / 1757 \\
(15.9 \%)\end{array}$ & $\begin{array}{c}12.47- \\
53.53\end{array}$ & $\begin{array}{c}95 / 875 \\
(10.9 \%)\end{array}$ & $1.78-6.1$ & 0.745 & $\begin{array}{c}140 / 875 \\
(16 \%)\end{array}$ & $\begin{array}{c}12.31- \\
55.37\end{array}$ & 0.9815 \\
\hline $\begin{array}{l}\text { Parathyroid } \\
\text { Hormone }\end{array}$ & $\begin{array}{c}27 / 1508 \\
(1.8 \%)\end{array}$ & $7-14.1$ & $\begin{array}{c}65 / 1508 \\
(4.3 \%)\end{array}$ & $65.6-108.9$ & $\begin{array}{l}6 / 751 \\
(0.8 \%)\end{array}$ & $\begin{array}{c}10.19- \\
14.37\end{array}$ & 0.0961 & $\begin{array}{l}31 / 751 \\
(4.1 \%)\end{array}$ & $66-498.9$ & 0.9268 \\
\hline Estradiol & N/A & N/A & N/A & N/A & N/A & N/A & N/A & N/A & N/A & N/A \\
\hline FSH & N/A & N/A & N/A & N/A & N/A & N/A & N/A & N/A & N/A & N/A \\
\hline $\mathrm{LH}$ & N/A & N/A & N/A & N/A & N/A & N/A & N/A & N/A & N/A & N/A \\
\hline Progesterone & N/A & N/A & N/A & N/A & N/A & N/A & N/A & N/A & N/A & N/A \\
\hline $\begin{array}{c}\text { Total } \\
\text { Testosterone }\end{array}$ & $\begin{array}{c}270 / 1810 \\
(14.9 \%)\end{array}$ & $2.52-121.6$ & $\begin{array}{c}155 / 1810 \\
(8.6 \%)\end{array}$ & $48.2-695.6$ & $\begin{array}{l}137 / 871 \\
(15.7 \%)\end{array}$ & $2.62-94.9$ & 0.6233 & $\begin{array}{l}86 / 871 \\
(9.9 \%)\end{array}$ & $\begin{array}{c}48.62- \\
596.5\end{array}$ & 0.299 \\
\hline SHBG & $\begin{array}{c}46 / 1754 \\
(2.6 \%)\end{array}$ & $7.8-24.23$ & $\begin{array}{c}495 / 1754 \\
(28.2 \%)\end{array}$ & $\begin{array}{c}122.3- \\
844.9\end{array}$ & $\begin{array}{l}28 / 847 \\
(3.3 \%)\end{array}$ & $7.72-24.5$ & 0.3918 & $\begin{array}{l}242 / 847 \\
(28.6 \%)\end{array}$ & $122.2-585$ & 0.8892 \\
\hline DHEA-S & $\begin{array}{c}195 / 1773 \\
(11 \%)\end{array}$ & $3.52-159.3$ & $\begin{array}{c}196 / 1773 \\
(11.1 \%)\end{array}$ & $\begin{array}{c}340.2- \\
762.4\end{array}$ & $\begin{array}{l}106 / 854 \\
(12.4 \%)\end{array}$ & $16.01-128$ & 0.3172 & $\begin{array}{l}79 / 854 \\
(9.3 \%)\end{array}$ & $\begin{array}{c}340.5- \\
638.3\end{array}$ & 0.1781 \\
\hline IGF-I & $\begin{array}{l}23 / 631 \\
(3.6 \%)\end{array}$ & $38.6-96.01$ & $\begin{array}{l}43 / 631 \\
(6.8 \%)\end{array}$ & $\begin{array}{c}235- \\
518.51\end{array}$ & $\begin{array}{c}16 / 322 \\
(5 \%)\end{array}$ & $\begin{array}{c}16.78- \\
89.71\end{array}$ & 0.422 & $\begin{array}{l}15 / 322 \\
(4.7 \%)\end{array}$ & $\begin{array}{c}242- \\
585.18\end{array}$ & 0.2405 \\
\hline Estriol & $0 / 272(0 \%)$ & N/A & $\begin{array}{l}8 / 272 \\
(2.9 \%)\end{array}$ & $\begin{array}{c}392.6- \\
851.8\end{array}$ & $0 / 149(0 \%)$ & N/A & N/A & $\begin{array}{l}7 / 149 \\
(4.7 \%)\end{array}$ & $\begin{array}{c}465.2- \\
880.9\end{array}$ & 0.5125 \\
\hline Estrone & $\begin{array}{c}43 / 360 \\
(11.9 \%)\end{array}$ & $9.5-15.8$ & $\begin{array}{l}13 / 360 \\
(3.6 \%)\end{array}$ & $\begin{array}{c}191.02- \\
859.52\end{array}$ & $\begin{array}{c}31 / 189 \\
(16.4 \%)\end{array}$ & $9-16.4$ & 0.1863 & $\begin{array}{l}5 / 189 \\
(2.6 \%)\end{array}$ & $\begin{array}{c}227.3- \\
300.2\end{array}$ & 0.7253 \\
\hline Prolactin & $1 / 24(4.2 \%)$ & $4.78-4.78$ & $\begin{array}{c}7 / 24 \\
(29.2 \%)\end{array}$ & $\begin{array}{c}23.51- \\
42.19\end{array}$ & $0 / 16(0 \%)$ & N/A & N/A & $4 / 16(25 \%)$ & $\begin{array}{c}23.67- \\
26.78\end{array}$ & 1 \\
\hline
\end{tabular}


Table S13. Age group 36 - 49. Reproductive hormonal changes in women with hypothyroidism. The significance was not considered when the total number of subjects tested for each hormonal group is $\leq 10$.

\begin{tabular}{|c|c|c|c|c|c|c|c|c|c|c|}
\hline & \multicolumn{4}{|c|}{ Hypothyroidism $(\mathrm{n}=387)$} & \multicolumn{6}{|c|}{ Thyroid Negative $(\mathrm{n}=5320)$} \\
\hline & Reduced & $\begin{array}{c}\text { Range } \\
\text { (reduced) }\end{array}$ & Elevated & $\begin{array}{c}\text { Range } \\
\text { (elevated) }\end{array}$ & Reduced & $\begin{array}{c}\text { Range } \\
\text { (reduced) }\end{array}$ & $\begin{array}{c}\text { P value } \\
\text { (reduced) }\end{array}$ & Elevated & $\begin{array}{c}\text { Range } \\
\text { (elevated) }\end{array}$ & $\begin{array}{l}\text { P value } \\
\text { (elevated) }\end{array}$ \\
\hline Cortisol & $\begin{array}{c}35 / 347 \\
(10.1 \%)\end{array}$ & $0.9-6.1$ & $\begin{array}{c}44 / 347 \\
(12.7 \%)\end{array}$ & $\begin{array}{c}17.04- \\
39.08\end{array}$ & $\begin{array}{c}637 / 4718 \\
(13.5 \%)\end{array}$ & $0.365-6.14$ & 0.084 & $\begin{array}{c}361 / 4718 \\
(7.7 \%)\end{array}$ & $12.1-47.1$ & 0.0012 \\
\hline $\begin{array}{c}\text { Parathyroid } \\
\text { Hormone }\end{array}$ & $\begin{array}{l}1 / 293 \\
(0.3 \%)\end{array}$ & $\begin{array}{c}14.43- \\
14.43\end{array}$ & $\begin{array}{l}22 / 293 \\
(7.5 \%)\end{array}$ & $65.5-142.5$ & $\begin{array}{c}24 / 4012 \\
(0.6 \%)\end{array}$ & $8.6-14.3$ & 1 & $\begin{array}{c}264 / 4012 \\
(6.6 \%)\end{array}$ & $65.5-200.1$ & 0.621 \\
\hline Estradiol & N/A & N/A & N/A & N/A & N/A & N/A & 1 & N/A & N/A & 1 \\
\hline FSH & N/A & N/A & N/A & N/A & $0 / 1(0 \%)$ & N/A & 1 & $1 / 1(100 \%)$ & $\begin{array}{c}61.09- \\
61.09\end{array}$ & 1 \\
\hline LH & N/A & N/A & N/A & N/A & N/A & N/A & 1 & N/A & N/A & 1 \\
\hline Progesterone & N/A & N/A & N/A & N/A & N/A & N/A & 1 & N/A & N/A & 1 \\
\hline $\begin{array}{c}\text { Total } \\
\text { Testosterone }\end{array}$ & $\begin{array}{l}100 / 351 \\
(28.5 \%)\end{array}$ & $2.5-59.5$ & $\begin{array}{l}17 / 351 \\
(4.8 \%)\end{array}$ & $48.6-523.1$ & $\begin{array}{c}1257 / 4944 \\
(25.4 \%)\end{array}$ & $2.5-159.2$ & 0.2271 & $\begin{array}{c}370 / 4944 \\
(7.5 \%)\end{array}$ & $\begin{array}{c}48.18- \\
1010\end{array}$ & 0.0835 \\
\hline SHBG & $\begin{array}{l}20 / 347 \\
(5.8 \%)\end{array}$ & $6.5-24.2$ & $\begin{array}{c}67 / 347 \\
(19.3 \%)\end{array}$ & $123.1-476$ & $\begin{array}{c}120 / 4806 \\
(2.5 \%)\end{array}$ & $7-24.5$ & 0.0006 & $\begin{array}{c}1172 / 4806 \\
(24.4 \%)\end{array}$ & $\begin{array}{c}122.1- \\
637.8\end{array}$ & 0.0382 \\
\hline DHEA-S & $\begin{array}{c}35 / 347 \\
(10.1 \%)\end{array}$ & $0.1-79.3$ & $\begin{array}{l}13 / 347 \\
(3.7 \%)\end{array}$ & $\begin{array}{c}256.4- \\
641.5\end{array}$ & $\begin{array}{c}296 / 4761 \\
(6.2 \%)\end{array}$ & $3.34-94.4$ & 0.0067 & $\begin{array}{c}244 / 4761 \\
(5.1 \%)\end{array}$ & $\begin{array}{c}256.6- \\
881.1\end{array}$ & 0.3139 \\
\hline IGF-I & $\begin{array}{l}10 / 122 \\
(8.2 \%)\end{array}$ & $35.51-70.2$ & $\begin{array}{c}2 / 122 \\
(1.6 \%)\end{array}$ & $\begin{array}{c}246- \\
387.08\end{array}$ & $\begin{array}{c}71 / 1948 \\
(3.6 \%)\end{array}$ & $\begin{array}{c}21.06- \\
97.02\end{array}$ & 0.0229 & $\begin{array}{c}64 / 1948 \\
(3.3 \%)\end{array}$ & $198-467$ & 0.4309 \\
\hline Estriol & $0 / 50(0 \%)$ & N/A & $2 / 50(4 \%)$ & $\begin{array}{c}300.7- \\
305.8\end{array}$ & $0 / 713(0 \%)$ & N/A & 1 & $\begin{array}{l}12 / 713 \\
(1.7 \%)\end{array}$ & $\begin{array}{c}390.3- \\
813.5\end{array}$ & 0.2322 \\
\hline Estrone & $7 / 71(9.9 \%)$ & $9.3-16.2$ & $0 / 71(0 \%)$ & N/A & $\begin{array}{c}150 / 1084 \\
(13.8 \%)\end{array}$ & $9.1-16.5$ & 0.442 & $\begin{array}{c}22 / 1084 \\
(2 \%)\end{array}$ & $\begin{array}{c}188.95- \\
734.5\end{array}$ & 0.3928 \\
\hline Prolactin & $0 / 3(0 \%)$ & N/A & $\begin{array}{c}1 / 3 \\
(33.3 \%)\end{array}$ & $\begin{array}{c}35.84- \\
35.84\end{array}$ & $3 / 89(3.4 \%)$ & $3.92-4.78$ & 1 & $\begin{array}{c}13 / 89 \\
(14.6 \%)\end{array}$ & $\begin{array}{c}24.28- \\
61.24\end{array}$ & 0.3942 \\
\hline
\end{tabular}

Table S14. Age group 36 - 49. Reproductive hormonal changes in women with hyperthyroidism. The significance was not considered when the total number of subjects tested for each hormonal group is $\leq 10$.

\begin{tabular}{|c|c|c|c|c|c|c|c|c|c|c|}
\hline & \multicolumn{4}{|c|}{ Hyperthyroidism $(\mathrm{n}=218)$} & \multicolumn{6}{|c|}{ Thyroid Negative $(\mathrm{n}=5320)$} \\
\hline & Reduced & $\begin{array}{c}\text { Range } \\
\text { (reduced) }\end{array}$ & Elevated & $\begin{array}{c}\text { Range } \\
\text { (elevated) }\end{array}$ & Reduced & $\begin{array}{c}\text { Range } \\
\text { (reduced) }\end{array}$ & $\begin{array}{l}\text { P value } \\
\text { (reduced) }\end{array}$ & Elevated & $\begin{array}{c}\text { Range } \\
\text { (elevated) }\end{array}$ & $\begin{array}{l}\text { P value } \\
\text { (elevated) }\end{array}$ \\
\hline Cortisol & $\begin{array}{c}22 / 176 \\
(12.5 \%)\end{array}$ & $0.837-6.13$ & $\begin{array}{l}17 / 176 \\
(9.7 \%)\end{array}$ & $\begin{array}{c}12.43- \\
36.47\end{array}$ & $\begin{array}{c}637 / 4718 \\
(13.5 \%)\end{array}$ & $0.365-6.14$ & 0.7874 & $\begin{array}{c}361 / 4718 \\
(7.7 \%)\end{array}$ & $12.1-47.1$ & 0.4033 \\
\hline $\begin{array}{c}\text { Parathyroid } \\
\text { Hormone }\end{array}$ & $\begin{array}{l}1 / 148 \\
(0.7 \%)\end{array}$ & $\begin{array}{c}12.44- \\
12.44\end{array}$ & $\begin{array}{l}5 / 148 \\
(3.4 \%)\end{array}$ & $\begin{array}{c}71.49- \\
203.7\end{array}$ & $\begin{array}{c}24 / 4012 \\
(0.6 \%)\end{array}$ & $8.6-14.3$ & 0.5968 & $\begin{array}{c}264 / 4012 \\
(6.6 \%)\end{array}$ & $65.5-200.1$ & 0.166 \\
\hline Estradiol & $\mathrm{N} / \mathrm{A}$ & N/A & $\mathrm{N} / \mathrm{A}$ & N/A & N/A & N/A & 1 & N/A & N/A & 1 \\
\hline FSH & N/A & N/A & N/A & N/A & $0 / 1(0 \%)$ & N/A & 1 & $1 / 1(100 \%)$ & $\begin{array}{c}61.09- \\
61.09\end{array}$ & 1 \\
\hline $\mathrm{LH}$ & N/A & N/A & N/A & N/A & N/A & N/A & 1 & N/A & N/A & 1 \\
\hline Progesterone & N/A & N/A & N/A & N/A & N/A & N/A & 1 & N/A & N/A & 1 \\
\hline
\end{tabular}


Continued

\begin{tabular}{|c|c|c|c|c|c|c|c|c|c|c|}
\hline $\begin{array}{c}\text { Total } \\
\text { Testosterone }\end{array}$ & $\begin{array}{l}43 / 205 \\
(21 \%)\end{array}$ & $2.68-134.9$ & $\begin{array}{c}40 / 205 \\
(19.5 \%)\end{array}$ & $\begin{array}{c}52.23- \\
460.1\end{array}$ & $\begin{array}{c}1257 / 4944 \\
(25.4 \%)\end{array}$ & $2.5-159.2$ & 0.1755 & $\begin{array}{c}370 / 4944 \\
(7.5 \%)\end{array}$ & $48.18-1010$ & 0 \\
\hline SHBG & $2 / 194(1 \%)$ & $12.3-22.5$ & $\begin{array}{c}70 / 194 \\
(36.1 \%)\end{array}$ & $\begin{array}{c}122.1- \\
432.1\end{array}$ & $\begin{array}{c}120 / 4806 \\
(2.5 \%)\end{array}$ & $7-24.5$ & 0.3347 & $\begin{array}{c}1172 / 4806 \\
(24.4 \%)\end{array}$ & $\begin{array}{c}122.1- \\
637.8\end{array}$ & 0.0003 \\
\hline DHEA-S & $\begin{array}{l}12 / 195 \\
(6.2 \%)\end{array}$ & $\begin{array}{c}15.74- \\
58.19\end{array}$ & $\begin{array}{l}14 / 195 \\
(7.2 \%)\end{array}$ & $\begin{array}{c}269.1- \\
579.1\end{array}$ & $\begin{array}{c}296 / 4761 \\
(6.2 \%)\end{array}$ & $3.34-94.4$ & 1 & $\begin{array}{c}244 / 4761 \\
(5.1 \%)\end{array}$ & $\begin{array}{c}256.6- \\
881.1\end{array}$ & 0.2707 \\
\hline IGF-I & $2 / 68(2.9 \%)$ & $\begin{array}{c}55.56- \\
96.63\end{array}$ & $\begin{array}{c}3 / 68 \\
(4.4 \%)\end{array}$ & $210-352.22$ & $\begin{array}{c}71 / 1948 \\
(3.6 \%)\end{array}$ & $\begin{array}{c}21.06- \\
97.02\end{array}$ & 1 & $\begin{array}{c}64 / 1948 \\
(3.3 \%)\end{array}$ & $198-467$ & 0.492 \\
\hline Estriol & $0 / 16(0 \%)$ & N/A & $0 / 16(0 \%)$ & N/A & 0/713 (0\%) & N/A & 1 & $\begin{array}{l}12 / 713 \\
(1.7 \%)\end{array}$ & $\begin{array}{c}390.3- \\
813.5\end{array}$ & 1 \\
\hline Estrone & $\begin{array}{c}5 / 46 \\
(10.9 \%)\end{array}$ & $11.2-14.5$ & $\begin{array}{c}1 / 46 \\
(2.2 \%)\end{array}$ & $\begin{array}{c}493.4- \\
493.4\end{array}$ & $\begin{array}{c}150 / 1084 \\
(13.8 \%)\end{array}$ & $9.1-16.5$ & 0.7231 & $\begin{array}{c}22 / 1084 \\
(2 \%)\end{array}$ & $\begin{array}{c}188.95- \\
734.5\end{array}$ & 0.6192 \\
\hline Prolactin & $0 / 3(0 \%)$ & N/A & $0 / 3(0 \%)$ & N/A & $3 / 89(3.4 \%)$ & $3.92-4.78$ & 1 & $\begin{array}{c}13 / 89 \\
(14.6 \%)\end{array}$ & $\begin{array}{c}24.28- \\
61.24\end{array}$ & 1 \\
\hline
\end{tabular}

Table S15. Age group 36 - 49. Reproductive hormonal changes in women with positive anti-TPO. The significance was not considered when the total number of subjects tested for each hormonal group is $\leq 10$.

\begin{tabular}{|c|c|c|c|c|c|c|c|c|c|c|}
\hline & \multicolumn{4}{|c|}{ Anti-TPO+ $(\mathrm{n}=5050)$} & \multicolumn{6}{|c|}{ Anti-TPO- $(\mathrm{n}=1145)$} \\
\hline & Reduced & $\begin{array}{c}\text { Range } \\
\text { (reduced) }\end{array}$ & Elevated & $\begin{array}{c}\text { Range } \\
\text { (elevated) }\end{array}$ & Reduced & $\begin{array}{c}\text { Range } \\
\text { (reduced) }\end{array}$ & $\begin{array}{c}\text { P value } \\
\text { (reduced) }\end{array}$ & Elevated & $\begin{array}{c}\text { Range } \\
\text { (elevated) }\end{array}$ & $\begin{array}{l}\text { P value } \\
\text { (elevated) }\end{array}$ \\
\hline Cortisol & $\begin{array}{c}604 / 4430 \\
(13.6 \%)\end{array}$ & $0.365-6.14$ & $\begin{array}{c}351 / 4430 \\
(7.9 \%)\end{array}$ & $12.1-45.3$ & $\begin{array}{c}120 / 1047 \\
(11.5 \%)\end{array}$ & $0.7-6.1$ & 0.0693 & $\begin{array}{c}89 / 1047 \\
(8.5 \%)\end{array}$ & $12.5-47.1$ & 0.579 \\
\hline $\begin{array}{c}\text { Parathyroid } \\
\text { Hormone }\end{array}$ & $\begin{array}{c}21 / 3718 \\
(0.6 \%)\end{array}$ & $8.6-14.43$ & $\begin{array}{c}229 / 3718 \\
(6.2 \%)\end{array}$ & $65.5-185.7$ & $\begin{array}{l}7 / 930 \\
(0.8 \%)\end{array}$ & $12.6-14.3$ & 0.6706 & $\begin{array}{l}78 / 930 \\
(8.4 \%)\end{array}$ & $65.5-203.7$ & 0.0177 \\
\hline Estradiol & N/A & N/A & N/A & N/A & N/A & N/A & 1 & N/A & N/A & 1 \\
\hline FSH & $0 / 1(0 \%)$ & N/A & $1 / 1(100 \%)$ & $\begin{array}{c}61.09- \\
61.09\end{array}$ & N/A & N/A & 1 & N/A & N/A & 1 \\
\hline LH & N/A & N/A & N/A & N/A & N/A & N/A & 1 & N/A & N/A & 1 \\
\hline Progesterone & N/A & N/A & N/A & N/A & N/A & N/A & 1 & N/A & N/A & 1 \\
\hline $\begin{array}{c}\text { Total } \\
\text { Testosterone }\end{array}$ & $\begin{array}{c}1093 / 4666 \\
(23.4 \%)\end{array}$ & $2.5-134.9$ & $\begin{array}{c}383 / 4666 \\
(8.2 \%)\end{array}$ & $\begin{array}{c}48.18- \\
1010\end{array}$ & $\begin{array}{c}346 / 1075 \\
(32.2 \%)\end{array}$ & $2.56-159.2$ & 0 & $\begin{array}{c}72 / 1075 \\
(6.7 \%)\end{array}$ & $48.2-624.3$ & 0.1118 \\
\hline SHBG & $\begin{array}{c}128 / 4538 \\
(2.8 \%)\end{array}$ & $6.5-24.4$ & $\begin{array}{c}1091 / 4538 \\
(24 \%)\end{array}$ & $\begin{array}{c}122.1- \\
637.8\end{array}$ & $\begin{array}{c}28 / 1057 \\
(2.6 \%)\end{array}$ & $7.5-24.5$ & 0.8403 & $\begin{array}{c}285 / 1057 \\
(27 \%)\end{array}$ & $\begin{array}{c}122.1- \\
552.5\end{array}$ & 0.0516 \\
\hline DHEA-S & $\begin{array}{c}298 / 4504 \\
(6.6 \%)\end{array}$ & $0.1-90.3$ & $\begin{array}{c}247 / 4504 \\
(5.5 \%)\end{array}$ & $\begin{array}{c}256.4- \\
881.1\end{array}$ & $\begin{array}{c}69 / 1044 \\
(6.6 \%)\end{array}$ & $5.9-94.4$ & 1 & $\begin{array}{c}36 / 1044 \\
(3.4 \%)\end{array}$ & $\begin{array}{c}261.6- \\
536.5\end{array}$ & 0.0089 \\
\hline IGF-I & $\begin{array}{c}53 / 1760 \\
(3 \%)\end{array}$ & $\begin{array}{c}31.53- \\
97.02\end{array}$ & $\begin{array}{c}58 / 1760 \\
(3.3 \%)\end{array}$ & $198-467$ & $\begin{array}{l}32 / 482 \\
(6.6 \%)\end{array}$ & $\begin{array}{c}21.06- \\
96.63\end{array}$ & 0.0004 & $\begin{array}{l}15 / 482 \\
(3.1 \%)\end{array}$ & $\begin{array}{c}221- \\
432.18\end{array}$ & 0.9552 \\
\hline Estriol & $0 / 726(0 \%)$ & N/A & $\begin{array}{l}12 / 726 \\
(1.7 \%)\end{array}$ & $\begin{array}{c}300.7- \\
813.5\end{array}$ & $0 / 91(0 \%)$ & N/A & 1 & $\begin{array}{c}5 / 91 \\
(5.5 \%)\end{array}$ & $\begin{array}{c}305.8- \\
438.8\end{array}$ & 0.0423 \\
\hline Estrone & $\begin{array}{c}156 / 1137 \\
(13.7 \%)\end{array}$ & $9.1-16.5$ & $\begin{array}{c}19 / 1137 \\
(1.7 \%)\end{array}$ & $\begin{array}{c}189.6- \\
734.5\end{array}$ & $\begin{array}{c}13 / 123 \\
(10.6 \%)\end{array}$ & $9.3-16.44$ & 0.4038 & $\begin{array}{l}5 / 123 \\
(4.1 \%)\end{array}$ & $\begin{array}{c}188.95- \\
374.4\end{array}$ & 0.1342 \\
\hline Prolactin & $2 / 89(2.2 \%)$ & $3.92-4.78$ & $\begin{array}{c}14 / 89 \\
(15.7 \%)\end{array}$ & $\begin{array}{c}24.28- \\
61.24\end{array}$ & $1 / 10(10 \%)$ & $4.62-4.62$ & 0.276 & $0 / 10(0 \%)$ & N/A & 0.3489 \\
\hline
\end{tabular}


Table S16. Age group 36 - 49. Reproductive hormonal changes in women with positive anti-Tg. The significance was not considered when the total number of subjects tested for each hormonal group is $\leq 10$.

\begin{tabular}{|c|c|c|c|c|c|c|c|c|c|c|}
\hline & \multicolumn{4}{|c|}{ Anti-Tg+ $(\mathrm{n}=4157)$} & \multicolumn{6}{|c|}{ Anti-Tg- $(\mathrm{n}=2040)$} \\
\hline & Reduced & $\begin{array}{c}\text { Range } \\
\text { (reduced) }\end{array}$ & Elevated & $\begin{array}{c}\text { Range } \\
\text { (elevated) }\end{array}$ & Reduced & $\begin{array}{c}\text { Range } \\
\text { (reduced) }\end{array}$ & $\begin{array}{l}\text { P value } \\
\text { (reduced) }\end{array}$ & Elevated & $\begin{array}{c}\text { Range } \\
\text { (elevated) }\end{array}$ & $\begin{array}{c}\text { P value } \\
\text { (elevated) }\end{array}$ \\
\hline Cortisol & $\begin{array}{c}492 / 3655 \\
(13.5 \%)\end{array}$ & $0.365-6.14$ & $\begin{array}{c}296 / 3655 \\
(8.1 \%)\end{array}$ & $12.1-47.1$ & $\begin{array}{c}232 / 1823 \\
(12.7 \%)\end{array}$ & $0.681-6.13$ & 0.475 & $\begin{array}{c}144 / 1823 \\
(7.9 \%)\end{array}$ & $12.1-42.15$ & 0.839 \\
\hline $\begin{array}{l}\text { Parathyroid } \\
\text { Hormone }\end{array}$ & $\begin{array}{c}23 / 3123 \\
(0.7 \%)\end{array}$ & $8.6-14.43$ & $\begin{array}{c}202 / 3123 \\
(6.5 \%)\end{array}$ & $65.5-203.7$ & $\begin{array}{l}5 / 1526 \\
(0.3 \%)\end{array}$ & $8.9-14.17$ & 0.1363 & $\begin{array}{c}105 / 1526 \\
(6.9 \%)\end{array}$ & $\begin{array}{c}65.59- \\
200.1\end{array}$ & 0.639 \\
\hline Estradiol & N/A & N/A & N/A & N/A & N/A & N/A & 1 & N/A & N/A & 1 \\
\hline FSH & $0 / 1(0 \%)$ & N/A & $1 / 1(100 \%)$ & $61.09-61.09$ & N/A & N/A & 1 & N/A & N/A & 1 \\
\hline LH & N/A & N/A & N/A & N/A & N/A & N/A & 1 & N/A & N/A & 1 \\
\hline Progesterone & N/A & N/A & N/A & N/A & N/A & N/A & 1 & N/A & N/A & 1 \\
\hline $\begin{array}{c}\text { Total } \\
\text { Testosterone }\end{array}$ & $\begin{array}{c}945 / 3856 \\
(24.5 \%)\end{array}$ & $2.5-159.2$ & $\begin{array}{c}302 / 3856 \\
(7.8 \%)\end{array}$ & $48.18-912.2$ & $\begin{array}{c}493 / 1885 \\
(26.2 \%)\end{array}$ & $2.5-153.9$ & 0.1869 & $\begin{array}{c}153 / 1885 \\
(8.1 \%)\end{array}$ & $48.2-1010$ & 0.7466 \\
\hline SHBG & $\begin{array}{c}105 / 3763 \\
(2.8 \%)\end{array}$ & $6.5-24.29$ & $\begin{array}{c}914 / 3763 \\
(24.3 \%)\end{array}$ & $122.1-637.8$ & $\begin{array}{c}51 / 1832 \\
(2.8 \%)\end{array}$ & $9.4-24.5$ & 1 & $\begin{array}{c}462 / 1832 \\
(25.2 \%)\end{array}$ & $\begin{array}{c}122.1- \\
552.5\end{array}$ & 0.4689 \\
\hline DHEA-S & $\begin{array}{c}253 / 3726 \\
(6.8 \%)\end{array}$ & $0.1-90.3$ & $\begin{array}{c}201 / 3726 \\
(5.4 \%)\end{array}$ & $256.4-881.1$ & $\begin{array}{c}114 / 1823 \\
(6.3 \%)\end{array}$ & $0.109-94.4$ & 0.4851 & $\begin{array}{c}82 / 1823 \\
(4.5 \%)\end{array}$ & $\begin{array}{c}258.4- \\
703.4\end{array}$ & 0.1736 \\
\hline IGF-I & $\begin{array}{c}56 / 1510 \\
(3.7 \%)\end{array}$ & $\begin{array}{c}21.06- \\
97.02\end{array}$ & $\begin{array}{c}48 / 1510 \\
(3.2 \%)\end{array}$ & $198-467$ & $29 / 732(4 \%)$ & $36.3-96.63$ & 0.86 & $\begin{array}{l}25 / 732 \\
(3.4 \%)\end{array}$ & $\begin{array}{c}198- \\
432.18\end{array}$ & 0.8658 \\
\hline Estriol & $0 / 521(0 \%)$ & N/A & $\begin{array}{l}11 / 521 \\
(2.1 \%)\end{array}$ & $300.7-813.5$ & 0/297 (0\%) & N/A & 1 & $6 / 297(2 \%)$ & $\begin{array}{c}305.8- \\
669.8\end{array}$ & 1 \\
\hline Estrone & $\begin{array}{c}98 / 818 \\
(12 \%)\end{array}$ & $9.1-16.2$ & $\begin{array}{l}12 / 818 \\
(1.5 \%)\end{array}$ & $\begin{array}{c}188.95- \\
734.5\end{array}$ & $\begin{array}{c}72 / 444 \\
(16.2 \%)\end{array}$ & $9.1-16.5$ & 0.0436 & $\begin{array}{l}12 / 444 \\
(2.7 \%)\end{array}$ & $\begin{array}{c}190.99- \\
625.31\end{array}$ & 0.1872 \\
\hline Prolactin & $1 / 60(1.7 \%)$ & $4.78-4.78$ & $\begin{array}{c}8 / 60 \\
(13.3 \%)\end{array}$ & $24.64-61.24$ & $2 / 39(5.1 \%)$ & $3.92-4.62$ & 0.5599 & $\begin{array}{c}6 / 39 \\
(15.4 \%)\end{array}$ & $\begin{array}{c}24.28- \\
49.21\end{array}$ & 1 \\
\hline
\end{tabular}

Table S17. Age group 15 - 49. Reproductive hormonal changes in men with hypothyroidism. The significance was not considered when the total number of subjects tested for each hormonal group is $\leq 10$.

\begin{tabular}{|c|c|c|c|c|c|c|c|c|c|c|}
\hline & \multicolumn{4}{|c|}{ Hypothyroidism $(\mathrm{n}=255)$} & \multicolumn{6}{|c|}{ Thyroid Negative $(\mathrm{n}=3982)$} \\
\hline & Reduced & $\begin{array}{c}\text { Range } \\
\text { (reduced) }\end{array}$ & Elevated & $\begin{array}{c}\text { Range } \\
\text { (elevated) }\end{array}$ & Reduced & $\begin{array}{c}\text { Range } \\
\text { (reduced) }\end{array}$ & $\begin{array}{c}\text { P value } \\
\text { (reduced) }\end{array}$ & Elevated & $\begin{array}{c}\text { Range } \\
\text { (elevated) }\end{array}$ & $\begin{array}{l}\text { P value } \\
\text { (elevated) }\end{array}$ \\
\hline Cortisol & $\begin{array}{c}26 / 222 \\
(11.7 \%)\end{array}$ & $1.06-6.09$ & $\begin{array}{c}20 / 222 \\
(9 \%)\end{array}$ & $15-32.5$ & $\begin{array}{c}390 / 3504 \\
(11.1 \%)\end{array}$ & $0.61-6.13$ & 0.8753 & $\begin{array}{c}245 / 3504 \\
(7 \%)\end{array}$ & $11.98-40.8$ & 0.3177 \\
\hline $\begin{array}{c}\text { Parathyroid } \\
\text { Hormone }\end{array}$ & $\begin{array}{c}3 / 192 \\
(1.6 \%)\end{array}$ & $8.8-13.53$ & $\begin{array}{c}6 / 192 \\
(3.1 \%)\end{array}$ & $\begin{array}{c}65.84- \\
101.5\end{array}$ & $\begin{array}{c}37 / 3004 \\
(1.2 \%)\end{array}$ & $8.36-14.3$ & 0.7314 & $\begin{array}{c}113 / 3004 \\
(3.8 \%)\end{array}$ & $\begin{array}{c}65.64- \\
465.4\end{array}$ & 0.7986 \\
\hline Estradiol & $\begin{array}{l}152 / 222 \\
(68.5 \%)\end{array}$ & $5.2-25.52$ & $\begin{array}{l}7 / 222 \\
(3.2 \%)\end{array}$ & $62.4-90.1$ & $\begin{array}{c}2392 / 3540 \\
(67.6 \%)\end{array}$ & $5.02-25.74$ & 0.8388 & $\begin{array}{c}72 / 3540 \\
(2 \%)\end{array}$ & $\begin{array}{c}61.22- \\
247.4\end{array}$ & 0.3751 \\
\hline FSH & $\begin{array}{l}29 / 206 \\
(14.1 \%)\end{array}$ & $0.1-1.42$ & $\begin{array}{l}6 / 206 \\
(2.9 \%)\end{array}$ & $12.7-34.04$ & $\begin{array}{c}451 / 3291 \\
(13.7 \%)\end{array}$ & $0.1-1.44$ & 0.9627 & $\begin{array}{c}58 / 3291 \\
(1.8 \%)\end{array}$ & $12.47-63.9$ & 0.354 \\
\hline LH & $\begin{array}{c}33 / 207 \\
(15.9 \%)\end{array}$ & $0.1-1.59$ & $\begin{array}{l}13 / 207 \\
(6.3 \%)\end{array}$ & $8.75-21.3$ & $\begin{array}{c}425 / 3325 \\
(12.8 \%)\end{array}$ & $0.1-1.64$ & 0.2276 & $\begin{array}{c}186 / 3325 \\
(5.6 \%)\end{array}$ & $8.68-53.67$ & 0.7948 \\
\hline Progesterone & $\begin{array}{l}59 / 198 \\
(29.8 \%)\end{array}$ & $\begin{array}{c}0.059- \\
0.191\end{array}$ & $\begin{array}{l}9 / 198 \\
(4.5 \%)\end{array}$ & $0.176-3.01$ & $\begin{array}{c}742 / 3124 \\
(23.8 \%)\end{array}$ & $\begin{array}{c}0.052- \\
0.194\end{array}$ & 0.0653 & $\begin{array}{c}146 / 3124 \\
(4.7 \%)\end{array}$ & $0.15-17.43$ & 1 \\
\hline
\end{tabular}


Continued

\begin{tabular}{|c|c|c|c|c|c|c|c|c|c|c|}
\hline $\begin{array}{c}\text { Total } \\
\text { Testosterone }\end{array}$ & $\begin{array}{c}46 / 249 \\
(18.5 \%)\end{array}$ & $\begin{array}{c}16.75- \\
334.6\end{array}$ & $\begin{array}{l}18 / 249 \\
(7.2 \%)\end{array}$ & $\begin{array}{c}876.6- \\
1488\end{array}$ & $\begin{array}{c}573 / 3885 \\
(14.7 \%)\end{array}$ & $5.5-347.3$ & 0.1322 & $\begin{array}{c}320 / 3885 \\
(8.2 \%)\end{array}$ & $\begin{array}{c}837.7- \\
1499\end{array}$ & 0.6575 \\
\hline SHBG & $\begin{array}{l}22 / 239 \\
(9.2 \%)\end{array}$ & $3.9-16.3$ & $\begin{array}{c}31 / 239 \\
(13 \%)\end{array}$ & $\begin{array}{c}56.24- \\
118.4\end{array}$ & $\begin{array}{c}247 / 3762 \\
(6.6 \%)\end{array}$ & $3.6-16.44$ & 0.148 & $\begin{array}{c}607 / 3762 \\
(16.1 \%)\end{array}$ & $56-226.6$ & 0.2284 \\
\hline DHEA-S & $\begin{array}{l}13 / 228 \\
(5.7 \%)\end{array}$ & $\begin{array}{c}21.94- \\
187.1\end{array}$ & $\begin{array}{l}21 / 228 \\
(9.2 \%)\end{array}$ & $\begin{array}{c}375.2- \\
693.2\end{array}$ & $\begin{array}{c}154 / 3572 \\
(4.3 \%)\end{array}$ & $8-205.6$ & 0.4086 & $\begin{array}{c}423 / 3572 \\
(11.8 \%)\end{array}$ & $261.6-972$ & 0.2744 \\
\hline IGF-I & $8 / 98(8.2 \%)$ & $15.52-145$ & $\begin{array}{c}7 / 98 \\
(7.1 \%)\end{array}$ & $\begin{array}{c}235- \\
401.05\end{array}$ & $\begin{array}{c}59 / 1759 \\
(3.4 \%)\end{array}$ & $\begin{array}{c}11.99 \text { - } \\
99.36\end{array}$ & 0.0274 & $\begin{array}{c}105 / 1759 \\
(6 \%)\end{array}$ & $200-599$ & 0.7972 \\
\hline Estriol & $0 / 26(0 \%)$ & N/A & $0 / 26(0 \%)$ & N/A & $0 / 364(0 \%)$ & N/A & 1 & $\begin{array}{l}7 / 364 \\
(1.9 \%)\end{array}$ & $\begin{array}{c}208.4- \\
426.5\end{array}$ & 1 \\
\hline Estrone & $\begin{array}{c}4 / 37 \\
(10.8 \%)\end{array}$ & N/A & $\begin{array}{c}3 / 37 \\
(8.1 \%)\end{array}$ & $54.4-62.43$ & $\begin{array}{c}89 / 537 \\
(16.6 \%)\end{array}$ & $9-10.1$ & 0.4899 & $\begin{array}{l}17 / 537 \\
(3.2 \%)\end{array}$ & $51.2-88.6$ & 0.1316 \\
\hline Prolactin & $0 / 11(0 \%)$ & N/A & $\begin{array}{c}4 / 11 \\
(36.4 \%)\end{array}$ & $\begin{array}{c}20.38- \\
24.09\end{array}$ & $4 / 151(2.6 \%)$ & $1.91-4.01$ & 1 & $\begin{array}{c}23 / 151 \\
(15.2 \%)\end{array}$ & $\begin{array}{c}15.26- \\
35.85\end{array}$ & 0.0882 \\
\hline
\end{tabular}

Table S18. Age group 15 - 49. Reproductive hormonal changes in men with hyperthyroidism. The significance was not considered when the total number of subjects tested for each hormonal group is $\leq 10$.

\begin{tabular}{|c|c|c|c|c|c|c|c|c|c|c|}
\hline & \multicolumn{4}{|c|}{ Hyperthyroidism $(\mathrm{n}=38)$} & \multicolumn{6}{|c|}{ Thyroid Negative $(\mathrm{n}=3982)$} \\
\hline & Reduced & $\begin{array}{c}\text { Range } \\
\text { (reduced) }\end{array}$ & Elevated & $\begin{array}{c}\text { Range } \\
\text { (elevated) }\end{array}$ & Reduced & $\begin{array}{l}\text { Range } \\
\text { (reduced) }\end{array}$ & $\begin{array}{l}\text { P value } \\
\text { (reduced) }\end{array}$ & Elevated & $\begin{array}{c}\text { Range } \\
\text { (elevated) }\end{array}$ & $\begin{array}{c}\text { P value } \\
\text { (elevated) }\end{array}$ \\
\hline Cortisol & $\begin{array}{c}4 / 30 \\
(13.3 \%)\end{array}$ & $3.7-5.32$ & $\begin{array}{c}4 / 30 \\
(13.3 \%)\end{array}$ & $20.7-24.79$ & $\begin{array}{c}390 / 3504 \\
(11.1 \%)\end{array}$ & $0.61-6.13$ & 0.5704 & $\begin{array}{c}245 / 3504 \\
(7 \%)\end{array}$ & $11.98-40.8$ & 0.1568 \\
\hline $\begin{array}{c}\text { Parathyroid } \\
\text { Hormone }\end{array}$ & $\begin{array}{c}1 / 27 \\
(3.7 \%)\end{array}$ & $10.2-10.2$ & $\begin{array}{c}1 / 27 \\
(3.7 \%)\end{array}$ & $\begin{array}{c}78.32- \\
78.32\end{array}$ & $\begin{array}{c}37 / 3004 \\
(1.2 \%)\end{array}$ & $8.36-14.3$ & 0.2897 & $\begin{array}{c}113 / 3004 \\
(3.8 \%)\end{array}$ & $\begin{array}{c}65.64- \\
465.4\end{array}$ & 1 \\
\hline Estradiol & $\begin{array}{c}20 / 34 \\
(58.8 \%)\end{array}$ & $5.9-23.92$ & $\begin{array}{c}3 / 34 \\
(8.8 \%)\end{array}$ & $\begin{array}{c}61.58- \\
113.9\end{array}$ & $\begin{array}{c}2392 / 3540 \\
(67.6 \%)\end{array}$ & $5.02-25.74$ & 0.3683 & $\begin{array}{c}72 / 3540 \\
(2 \%)\end{array}$ & $\begin{array}{c}61.22- \\
247.4\end{array}$ & 0.0333 \\
\hline FSH & $\begin{array}{c}5 / 30 \\
(16.7 \%)\end{array}$ & $0.129-1$ & $\begin{array}{c}3 / 30 \\
(10 \%)\end{array}$ & $\begin{array}{c}12.93- \\
121.9\end{array}$ & $\begin{array}{c}451 / 3291 \\
(13.7 \%)\end{array}$ & $0.1-1.44$ & 0.8392 & $\begin{array}{c}58 / 3291 \\
(1.8 \%)\end{array}$ & $12.47-63.9$ & 0.0168 \\
\hline LH & $\begin{array}{c}6 / 30 \\
(20 \%)\end{array}$ & $0.3-1.38$ & $\begin{array}{c}6 / 30 \\
(20 \%)\end{array}$ & $8.7-61.24$ & $\begin{array}{c}425 / 3325 \\
(12.8 \%)\end{array}$ & $0.1-1.64$ & 0.367 & $\begin{array}{c}186 / 3325 \\
(5.6 \%)\end{array}$ & $8.68-53.67$ & 0.0028 \\
\hline Progesterone & $\begin{array}{c}8 / 28 \\
(28.6 \%)\end{array}$ & $\begin{array}{c}0.065- \\
0.182\end{array}$ & $\begin{array}{c}3 / 28 \\
(10.7 \%)\end{array}$ & $0.19-5.07$ & $\begin{array}{c}742 / 3124 \\
(23.8 \%)\end{array}$ & $\begin{array}{c}0.052- \\
0.194\end{array}$ & 0.7089 & $\begin{array}{c}146 / 3124 \\
(4.7 \%)\end{array}$ & $0.15-17.43$ & 0.1437 \\
\hline $\begin{array}{c}\text { Total } \\
\text { Testosterone }\end{array}$ & $\begin{array}{c}9 / 37 \\
(24.3 \%)\end{array}$ & $22.9-204.1$ & $\begin{array}{c}5 / 37 \\
(13.5 \%)\end{array}$ & $865.7-1400$ & $\begin{array}{c}573 / 3885 \\
(14.7 \%)\end{array}$ & $5.5-347.3$ & 0.162 & $\begin{array}{c}320 / 3885 \\
(8.2 \%)\end{array}$ & $837.7-1499$ & 0.3902 \\
\hline SHBG & $\begin{array}{c}3 / 37 \\
(8.1 \%)\end{array}$ & $8.5-13.7$ & $\begin{array}{c}14 / 37 \\
(37.8 \%)\end{array}$ & $56.6-189.5$ & $\begin{array}{c}247 / 3762 \\
(6.6 \%)\end{array}$ & $3.6-16.44$ & 0.7328 & $\begin{array}{c}607 / 3762 \\
(16.1 \%)\end{array}$ & $56-226.6$ & 0.0009 \\
\hline DHEA-S & $\begin{array}{c}6 / 35 \\
(17.1 \%)\end{array}$ & $\begin{array}{c}23.79- \\
153.7\end{array}$ & $\begin{array}{c}5 / 35 \\
(14.3 \%)\end{array}$ & $\begin{array}{c}364.2- \\
486.2\end{array}$ & $\begin{array}{c}154 / 3572 \\
(4.3 \%)\end{array}$ & $8-205.6$ & 0.0011 & $\begin{array}{c}423 / 3572 \\
(11.8 \%)\end{array}$ & $261.6-972$ & 0.8554 \\
\hline IGF-I & $\begin{array}{c}1 / 17 \\
(5.9 \%)\end{array}$ & $110-110$ & $\begin{array}{c}1 / 17 \\
(5.9 \%)\end{array}$ & $297-297$ & $\begin{array}{c}59 / 1759 \\
(3.4 \%)\end{array}$ & $\begin{array}{c}11.99- \\
99.36\end{array}$ & 0.444 & $\begin{array}{c}105 / 1759 \\
(6 \%)\end{array}$ & $200-599$ & 1 \\
\hline Estriol & $0 / 4(0 \%)$ & N/A & $0 / 4(0 \%)$ & $\mathrm{N} / \mathrm{A}$ & $0 / 364(0 \%)$ & N/A & 1 & $7 / 364$ (1.9\%) & $\begin{array}{c}208.4- \\
426.5\end{array}$ & 1 \\
\hline Estrone & $1 / 5(20 \%)$ & N/A & $0 / 5(0 \%)$ & N/A & $\begin{array}{c}89 / 537 \\
(16.6 \%)\end{array}$ & $9-10.1$ & 1 & $\begin{array}{l}17 / 537 \\
(3.2 \%)\end{array}$ & $51.2-88.6$ & 1 \\
\hline Prolactin & $0 / 2(0 \%)$ & N/A & $\begin{array}{c}2 / 2 \\
(100 \%)\end{array}$ & $\begin{array}{c}20.28- \\
21.01\end{array}$ & $4 / 151(2.6 \%)$ & $1.91-4.01$ & 1 & $\begin{array}{c}23 / 151 \\
(15.2 \%)\end{array}$ & $\begin{array}{c}15.26- \\
35.85\end{array}$ & 0.0258 \\
\hline
\end{tabular}


Table S19. Age group 15 - 49. Reproductive hormonal changes in men with positive anti-TPO. The significance was not considered when the total number of subjects tested for each hormonal group is $\leq 10$.

\begin{tabular}{|c|c|c|c|c|c|c|c|c|c|c|}
\hline & \multicolumn{4}{|c|}{ Anti-TPO+ $(\mathrm{n}=3284)$} & \multicolumn{6}{|c|}{ Anti-TPO- $(\mathrm{n}=1087)$} \\
\hline & Reduced & $\begin{array}{c}\text { Range } \\
\text { (reduced) }\end{array}$ & Elevated & $\begin{array}{c}\text { Range } \\
\text { (elevated) }\end{array}$ & Reduced & $\begin{array}{c}\text { Range } \\
\text { (reduced) }\end{array}$ & $\begin{array}{c}\text { P value } \\
\text { (reduced) }\end{array}$ & Elevated & $\begin{array}{c}\text { Range } \\
\text { (elevated) }\end{array}$ & $\begin{array}{l}\text { P value } \\
\text { (elevated) }\end{array}$ \\
\hline Cortisol & $\begin{array}{c}336 / 2870 \\
(11.7 \%)\end{array}$ & $0.61-6.13$ & $\begin{array}{c}160 / 2870 \\
(5.6 \%)\end{array}$ & $\begin{array}{c}12.21- \\
30.97\end{array}$ & $\begin{array}{l}90 / 954 \\
(9.4 \%)\end{array}$ & $0.7-6.12$ & 0.0609 & $\begin{array}{l}113 / 954 \\
(11.8 \%)\end{array}$ & $20.7-24.4$ & 0 \\
\hline $\begin{array}{l}\text { Parathyroid } \\
\text { Hormone }\end{array}$ & $28 / 2435(1.1 \%)$ & $\begin{array}{l}8.36- \\
14.29\end{array}$ & $\begin{array}{c}87 / 2435 \\
(3.6 \%)\end{array}$ & $\begin{array}{c}65.64- \\
465.4\end{array}$ & $\begin{array}{l}15 / 852 \\
(1.8 \%)\end{array}$ & $8.8-14.3$ & 0.24 & $\begin{array}{l}35 / 852 \\
(4.1 \%)\end{array}$ & $78.32-78.32$ & 0.5446 \\
\hline Estradiol & $\begin{array}{c}1930 / 2919 \\
(66.1 \%)\end{array}$ & $\begin{array}{l}5.02- \\
25.74\end{array}$ & $\begin{array}{c}71 / 2919 \\
(2.4 \%)\end{array}$ & $\begin{array}{c}61.22- \\
247.4\end{array}$ & $\begin{array}{l}689 / 964 \\
(71.5 \%)\end{array}$ & $5.2-25.7$ & 0.0024 & $\begin{array}{l}22 / 964 \\
(2.3 \%)\end{array}$ & $61.58-113.9$ & 0.8863 \\
\hline FSH & $\begin{array}{c}363 / 2666 \\
(13.6 \%)\end{array}$ & $0.1-1.44$ & $\begin{array}{c}54 / 2666 \\
(2 \%)\end{array}$ & $\begin{array}{c}12.47- \\
121.9\end{array}$ & $\begin{array}{l}141 / 915 \\
(15.4 \%)\end{array}$ & $0.1-1.4$ & 0.1966 & $\begin{array}{l}13 / 915 \\
(1.4 \%)\end{array}$ & $52.2-52.2$ & 0.3061 \\
\hline $\mathrm{LH}$ & $\begin{array}{c}339 / 2687 \\
(12.6 \%)\end{array}$ & $0.1-1.64$ & $\begin{array}{c}158 / 2687 \\
(5.9 \%)\end{array}$ & $8.68-61.24$ & $\begin{array}{l}143 / 929 \\
(15.4 \%)\end{array}$ & $0.1-1.6$ & 0.0366 & $\begin{array}{l}50 / 929 \\
(5.4 \%)\end{array}$ & $8.7-27.1$ & 0.631 \\
\hline Progesterone & $\begin{array}{c}657 / 2539 \\
(25.9 \%)\end{array}$ & $\begin{array}{c}0.052- \\
0.194\end{array}$ & $\begin{array}{c}138 / 2539 \\
(5.4 \%)\end{array}$ & $0.15-6.29$ & $\begin{array}{l}160 / 868 \\
(18.4 \%)\end{array}$ & $\begin{array}{c}0.053- \\
0.192\end{array}$ & 0 & $\begin{array}{l}32 / 868 \\
(3.7 \%)\end{array}$ & $0.212-0.212$ & 0.0509 \\
\hline $\begin{array}{c}\text { Total } \\
\text { Testosterone }\end{array}$ & $\begin{array}{c}425 / 3204 \\
(13.3 \%)\end{array}$ & $\begin{array}{l}10.3- \\
347.3\end{array}$ & $\begin{array}{c}275 / 3204 \\
(8.6 \%)\end{array}$ & $\begin{array}{c}837.7- \\
1499\end{array}$ & $\begin{array}{c}212 / 1057 \\
(20.1 \%)\end{array}$ & $5.5-345$ & 0 & $\begin{array}{c}81 / 1057 \\
(7.7 \%)\end{array}$ & $1013-1400$ & 0.3826 \\
\hline SHBG & $191 / 3102(6.2 \%)$ & $3.6-16.44$ & $\begin{array}{c}518 / 3102 \\
(16.7 \%)\end{array}$ & $56-189.5$ & $\begin{array}{c}89 / 1037 \\
(8.6 \%)\end{array}$ & $3.9-16.4$ & 0.0088 & $\begin{array}{c}144 / 1037 \\
(13.9 \%)\end{array}$ & $56.6-189.5$ & 0.0366 \\
\hline DHEA-S & $138 / 2930(4.7 \%)$ & $8-203.5$ & $\begin{array}{c}338 / 2930 \\
(11.5 \%)\end{array}$ & $\begin{array}{c}304.2- \\
955.3\end{array}$ & $\begin{array}{l}42 / 990 \\
(4.2 \%)\end{array}$ & $24.9-205.6$ & 0.6033 & $\begin{array}{l}124 / 990 \\
(12.5 \%)\end{array}$ & $364.2-486.2$ & 0.4367 \\
\hline IGF-I & 47/1374 (3.4\%) & $15.4-145$ & $\begin{array}{c}72 / 1374 \\
(5.2 \%)\end{array}$ & $200-599$ & $\begin{array}{l}25 / 534 \\
(4.7 \%)\end{array}$ & $\begin{array}{c}11.99- \\
99.36\end{array}$ & 0.2445 & $\begin{array}{l}44 / 534 \\
(8.2 \%)\end{array}$ & N/A & 0.0185 \\
\hline Estriol & $0 / 344(0 \%)$ & N/A & $\begin{array}{l}6 / 344 \\
(1.7 \%)\end{array}$ & $\begin{array}{c}208.4- \\
426.5\end{array}$ & $0 / 58(0 \%)$ & N/A & 1 & $1 / 58(1.7 \%)$ & N/A & 1 \\
\hline Estrone & $74 / 518(14.3 \%)$ & $9-10.1$ & $\begin{array}{l}18 / 518 \\
(3.5 \%)\end{array}$ & $51.3-88.6$ & $\begin{array}{c}23 / 77 \\
(29.9 \%)\end{array}$ & $9.1-10$ & 0.001 & $4 / 77(5.2 \%)$ & N/A & 0.5114 \\
\hline Prolactin & $3 / 145(2.1 \%)$ & $1.91-4.01$ & $\begin{array}{c}23 / 145 \\
(15.9 \%)\end{array}$ & $\begin{array}{c}15.26- \\
35.85\end{array}$ & $1 / 23(4.3 \%)$ & $3.6-3.6$ & 0.4483 & $\begin{array}{c}6 / 23 \\
(26.1 \%)\end{array}$ & $21.01-21.01$ & 0.3636 \\
\hline
\end{tabular}

Table S20. Age group 15 - 49. Reproductive hormonal changes in men with positive anti-Tg. The significance was not considered when the total number of subjects tested for each hormonal group is $\leq 10$.

\begin{tabular}{|c|c|c|c|c|c|c|c|c|c|c|}
\hline & \multicolumn{4}{|c|}{ Anti-Tg+ $(\mathrm{n}=2530)$} & \multicolumn{6}{|c|}{ Anti-Tg- $(\mathrm{n}=1846)$} \\
\hline & Reduced & $\begin{array}{c}\text { Range } \\
\text { (reduced) }\end{array}$ & Elevated & $\begin{array}{c}\text { Range } \\
\text { (elevated) }\end{array}$ & Reduced & $\begin{array}{c}\text { Range } \\
\text { (reduced) }\end{array}$ & $\begin{array}{l}\text { P value } \\
\text { (reduced) }\end{array}$ & Elevated & $\begin{array}{c}\text { Range } \\
\text { (elevated) }\end{array}$ & $\begin{array}{l}\text { P value } \\
\text { (elevated) }\end{array}$ \\
\hline Cortisol & $\begin{array}{c}238 / 2204 \\
(10.8 \%)\end{array}$ & $0.7-6.11$ & $\begin{array}{c}152 / 2204 \\
(6.9 \%)\end{array}$ & $11.98-40.8$ & $\begin{array}{c}189 / 1622 \\
(11.7 \%)\end{array}$ & $0.61-6.13$ & 0.4373 & $\begin{array}{c}121 / 1622 \\
(7.5 \%)\end{array}$ & $13.07-35.2$ & 0.5449 \\
\hline $\begin{array}{c}\text { Parathyroid } \\
\text { Hormone }\end{array}$ & $\begin{array}{c}29 / 1882 \\
(1.5 \%)\end{array}$ & $8.36-14.29$ & $\begin{array}{c}68 / 1882 \\
(3.6 \%)\end{array}$ & $\begin{array}{c}65.64- \\
465.4\end{array}$ & $\begin{array}{c}14 / 1407 \\
(1 \%)\end{array}$ & $8.8-14.3$ & 0.2269 & $\begin{array}{c}54 / 1407 \\
(3.8 \%)\end{array}$ & $\begin{array}{c}65.66- \\
138.4\end{array}$ & 0.8071 \\
\hline Estradiol & $\begin{array}{c}1518 / 2256 \\
(67.3 \%)\end{array}$ & $5.02-25.74$ & $\begin{array}{c}49 / 2256 \\
(2.2 \%)\end{array}$ & $\begin{array}{c}61.22- \\
316.7\end{array}$ & $\begin{array}{c}1103 / 1629 \\
(67.7 \%)\end{array}$ & $5.25-25.7$ & 0.808 & $\begin{array}{c}44 / 1629 \\
(2.7 \%)\end{array}$ & $\begin{array}{c}61.58- \\
189.8\end{array}$ & 0.338 \\
\hline FSH & $\begin{array}{c}309 / 2074 \\
(14.9 \%)\end{array}$ & $0.1-1.43$ & $\begin{array}{c}40 / 2074 \\
(1.9 \%)\end{array}$ & $12.5-121.9$ & $\begin{array}{c}195 / 1512 \\
(12.9 \%)\end{array}$ & $0.1-1.44$ & 0.098 & $\begin{array}{c}27 / 1512 \\
(1.8 \%)\end{array}$ & $12.47-63.9$ & 0.8514 \\
\hline $\mathrm{LH}$ & $\begin{array}{c}296 / 2088 \\
(14.2 \%)\end{array}$ & $0.1-1.62$ & $\begin{array}{c}119 / 2088 \\
(5.7 \%)\end{array}$ & $8.68-61.24$ & $\begin{array}{c}186 / 1533 \\
(12.1 \%)\end{array}$ & $0.1-1.64$ & 0.0821 & $\begin{array}{c}89 / 1533 \\
(5.8 \%)\end{array}$ & $8.7-53.49$ & 0.9492 \\
\hline
\end{tabular}




\section{Continued}

\begin{tabular}{|c|c|c|c|c|c|c|c|c|c|c|}
\hline Progesterone & $\begin{array}{c}392 / 1965 \\
(19.9 \%)\end{array}$ & $\begin{array}{c}0.052- \\
0.194\end{array}$ & $\begin{array}{c}111 / 1965 \\
(5.6 \%)\end{array}$ & $0.15-17.02$ & $\begin{array}{c}425 / 1445 \\
(29.4 \%)\end{array}$ & $\begin{array}{c}0.052- \\
0.194\end{array}$ & 0 & $\begin{array}{c}59 / 1445 \\
(4.1 \%)\end{array}$ & $\begin{array}{l}0.15- \\
17.43\end{array}$ & 0.0459 \\
\hline $\begin{array}{c}\text { Total } \\
\text { Testosterone }\end{array}$ & $\begin{array}{c}373 / 2476 \\
(15.1 \%)\end{array}$ & $5.5-345$ & $\begin{array}{c}208 / 2476 \\
(8.4 \%)\end{array}$ & $\begin{array}{c}843.5- \\
1499\end{array}$ & $\begin{array}{c}265 / 1789 \\
(14.8 \%)\end{array}$ & $10-347.3$ & 0.8539 & $\begin{array}{c}148 / 1789 \\
(8.3 \%)\end{array}$ & $\begin{array}{c}837.7- \\
1496\end{array}$ & 0.926 \\
\hline SHBG & $\begin{array}{c}160 / 2402 \\
(6.7 \%)\end{array}$ & $3.6-16.37$ & $\begin{array}{c}387 / 2402 \\
(16.1 \%)\end{array}$ & $\begin{array}{c}56.04- \\
226.6\end{array}$ & $\begin{array}{c}120 / 1740 \\
(6.9 \%)\end{array}$ & $4.2-16.44$ & 0.8141 & $\begin{array}{c}277 / 1740 \\
(15.9 \%)\end{array}$ & $56-189.5$ & 0.9018 \\
\hline DHEA-S & $\begin{array}{c}105 / 2280 \\
(4.6 \%)\end{array}$ & $\begin{array}{c}20.63- \\
192.8\end{array}$ & $\begin{array}{c}278 / 2280 \\
(12.2 \%)\end{array}$ & $331.6-972$ & $\begin{array}{c}75 / 1643 \\
(4.6 \%)\end{array}$ & $8-205.6$ & 1 & $\begin{array}{c}185 / 1643 \\
(11.3 \%)\end{array}$ & $\begin{array}{c}261.6- \\
922.3\end{array}$ & 0.3989 \\
\hline IGF-I & $\begin{array}{c}35 / 1142 \\
(3.1 \%)\end{array}$ & $11.99-110$ & $\begin{array}{c}60 / 1142 \\
(5.3 \%)\end{array}$ & $200-599$ & $\begin{array}{l}37 / 768 \\
(4.8 \%)\end{array}$ & $15.4-145$ & 0.0644 & $\begin{array}{l}56 / 768 \\
(7.3 \%)\end{array}$ & $205-597$ & 0.0835 \\
\hline Estriol & $0 / 235(0 \%)$ & N/A & $\begin{array}{l}4 / 235 \\
(1.7 \%)\end{array}$ & $\begin{array}{c}208.4- \\
426.5\end{array}$ & $0 / 167(0 \%)$ & N/A & 1 & $\begin{array}{c}3 / 167 \\
(1.8 \%)\end{array}$ & $\begin{array}{c}210.5- \\
236.8\end{array}$ & 1 \\
\hline Estrone & $\begin{array}{c}57 / 356 \\
(16 \%)\end{array}$ & $9-10.1$ & $\begin{array}{l}14 / 356 \\
(3.9 \%)\end{array}$ & $51.2-88.6$ & $\begin{array}{c}40 / 239 \\
(16.7 \%)\end{array}$ & $9.1-10$ & 0.9032 & $\begin{array}{l}8 / 239 \\
(3.3 \%)\end{array}$ & $51.3-87.4$ & 0.8813 \\
\hline Prolactin & $2 / 96(2.1 \%)$ & $3.69-4.01$ & $\begin{array}{c}17 / 96 \\
(17.7 \%)\end{array}$ & $\begin{array}{c}15.65- \\
35.85\end{array}$ & $2 / 72(2.8 \%)$ & $1.91-3.6$ & 1 & $\begin{array}{c}12 / 72 \\
(16.7 \%)\end{array}$ & $\begin{array}{c}15.26- \\
33.95\end{array}$ & 1 \\
\hline
\end{tabular}

Table S21. Age group 15 - 25. Reproductive hormonal changes in men with hypothyroidism. The significance was not considered when the total number of subjects tested for each hormonal group is $\leq 10$.

\begin{tabular}{|c|c|c|c|c|c|c|c|c|c|c|}
\hline & \multicolumn{4}{|c|}{ Hypothyroidism $(\mathrm{n}=47)$} & \multicolumn{6}{|c|}{ Thyroid Negative $(\mathrm{n}=559)$} \\
\hline & Reduced & $\begin{array}{c}\text { Range } \\
\text { (reduced) }\end{array}$ & Elevated & $\begin{array}{c}\text { Range } \\
\text { (elevated) }\end{array}$ & Reduced & $\begin{array}{c}\text { Range } \\
\text { (reduced) }\end{array}$ & $\begin{array}{l}\text { P value } \\
\text { (reduced) }\end{array}$ & Elevated & $\begin{array}{c}\text { Range } \\
\text { (elevated) }\end{array}$ & $\begin{array}{l}\text { P value } \\
\text { (elevated) }\end{array}$ \\
\hline Cortisol & $\begin{array}{c}6 / 43 \\
(14 \%)\end{array}$ & $3.54-5.81$ & $\begin{array}{c}4 / 43 \\
(9.3 \%)\end{array}$ & $20.11-32.5$ & $\begin{array}{c}58 / 508 \\
(11.4 \%)\end{array}$ & $0.61-6.1$ & 0.8022 & $\begin{array}{c}51 / 508 \\
(10 \%)\end{array}$ & $12.79-40.8$ & 1 \\
\hline $\begin{array}{c}\text { Parathyroid } \\
\text { Hormone }\end{array}$ & $\begin{array}{c}2 / 36 \\
(5.6 \%)\end{array}$ & $8.8-13.53$ & $\begin{array}{l}0 / 36 \\
(0 \%)\end{array}$ & N/A & $\begin{array}{l}11 / 448 \\
(2.5 \%)\end{array}$ & $10.5-14.3$ & 0.2508 & $\begin{array}{l}6 / 448 \\
(1.3 \%)\end{array}$ & $\begin{array}{c}66.33- \\
80.19\end{array}$ & 1 \\
\hline Estradiol & $\begin{array}{c}25 / 38 \\
(65.8 \%)\end{array}$ & $5.5-25.4$ & $\begin{array}{l}0 / 38 \\
(0 \%)\end{array}$ & N/A & $\begin{array}{l}359 / 489 \\
(73.4 \%)\end{array}$ & $5.25-25.7$ & 0.4071 & $0 / 489(0 \%)$ & N/A & 1 \\
\hline FSH & $\begin{array}{c}2 / 35 \\
(5.7 \%)\end{array}$ & $0.905-1.1$ & $\begin{array}{l}0 / 35 \\
(0 \%)\end{array}$ & N/A & $\begin{array}{l}38 / 456 \\
(8.3 \%)\end{array}$ & $0.117-1.43$ & 1 & $\begin{array}{c}2 / 456 \\
(0.4 \%)\end{array}$ & $14.24-20.3$ & 1 \\
\hline LH & $\begin{array}{c}1 / 36 \\
(2.8 \%)\end{array}$ & $1.4-1.4$ & $\begin{array}{c}1 / 36 \\
(2.8 \%)\end{array}$ & $9.9-9.9$ & $\begin{array}{l}18 / 459 \\
(3.9 \%)\end{array}$ & $0.116-1.6$ & 1 & $\begin{array}{l}24 / 459 \\
(5.2 \%)\end{array}$ & $8.69-13.53$ & 1 \\
\hline Progesterone & $\begin{array}{l}7 / 35 \\
(20 \%)\end{array}$ & $0.059-0.19$ & $\begin{array}{c}3 / 35 \\
(8.6 \%)\end{array}$ & $\begin{array}{c}0.307- \\
0.571\end{array}$ & $\begin{array}{c}56 / 454 \\
(12.3 \%)\end{array}$ & $\begin{array}{c}0.058- \\
0.194\end{array}$ & 0.2972 & $\begin{array}{l}35 / 454 \\
(7.7 \%)\end{array}$ & $0.15-1.69$ & 0.7461 \\
\hline $\begin{array}{c}\text { Total } \\
\text { Testosterone }\end{array}$ & $\begin{array}{c}10 / 43 \\
(23.3 \%)\end{array}$ & $48-332.2$ & $\begin{array}{l}0 / 43 \\
(0 \%)\end{array}$ & N/A & $\begin{array}{c}60 / 533 \\
(11.3 \%)\end{array}$ & $10,344.30$ & 0.0381 & $\begin{array}{l}24 / 533 \\
(4.5 \%)\end{array}$ & $837.7-1210$ & 0.2447 \\
\hline SHBG & $\begin{array}{c}6 / 40 \\
(15 \%)\end{array}$ & $5.57-14.46$ & $\begin{array}{c}3 / 40 \\
(7.5 \%)\end{array}$ & $59.85-73.8$ & $\begin{array}{l}37 / 510 \\
(7.3 \%)\end{array}$ & $6.4-16.4$ & 0.1467 & $\begin{array}{c}92 / 510 \\
(18 \%)\end{array}$ & $\begin{array}{c}56.04- \\
226.6\end{array}$ & 0.1255 \\
\hline DHEA-S & $\begin{array}{c}4 / 40 \\
(10 \%)\end{array}$ & $\begin{array}{c}68.57- \\
187.1\end{array}$ & $\begin{array}{c}6 / 40 \\
(15 \%)\end{array}$ & $\begin{array}{c}495.2- \\
693.2\end{array}$ & $\begin{array}{l}21 / 505 \\
(4.2 \%)\end{array}$ & $34.6-203.5$ & 0.1021 & $\begin{array}{c}74 / 505 \\
(14.7 \%)\end{array}$ & $\begin{array}{c}261.6- \\
955.3\end{array}$ & 1 \\
\hline IGF-I & $\begin{array}{c}3 / 20 \\
(15 \%)\end{array}$ & $39.7-145$ & $\begin{array}{c}4 / 20 \\
(20 \%)\end{array}$ & $\begin{array}{l}332.5- \\
401.05\end{array}$ & $\begin{array}{l}8 / 240 \\
(3.3 \%)\end{array}$ & $56.23-97.6$ & 0.0433 & $\begin{array}{c}42 / 240 \\
(17.5 \%)\end{array}$ & $303.55-599$ & 0.7622 \\
\hline Estriol & $0 / 4(0 \%)$ & N/A & $0 / 4(0 \%)$ & N/A & $0 / 70(0 \%)$ & N/A & 1 & $\begin{array}{c}1 / 70 \\
(1.4 \%)\end{array}$ & $\begin{array}{c}210.5- \\
210.5\end{array}$ & 1 \\
\hline Estrone & $1 / 4(25 \%)$ & N/A & $0 / 4(0 \%)$ & N/A & $\begin{array}{c}22 / 79 \\
(27.8 \%)\end{array}$ & $10-$ Sep & 1 & $0 / 79(0 \%)$ & N/A & 1 \\
\hline Prolactin & N/A & N/A & N/A & N/A & $1 / 15(6.7 \%)$ & $3.69-3.69$ & 1 & $\begin{array}{c}4 / 15 \\
(26.7 \%)\end{array}$ & $\begin{array}{c}15.26- \\
21.96\end{array}$ & 1 \\
\hline
\end{tabular}


Table S22. Age group 15 - 25. Reproductive hormonal changes in men with hyperthyroidism. The significance was not considered when the total number of subjects tested for each hormonal group is $\leq 10$.

\begin{tabular}{|c|c|c|c|c|c|c|c|c|c|c|}
\hline & \multicolumn{4}{|c|}{ Hyperthyroidism $(\mathrm{n}=3)$} & \multicolumn{6}{|c|}{ Thyroid Negative $(\mathrm{n}=559)$} \\
\hline & Reduced & $\begin{array}{c}\text { Range } \\
\text { (reduced) }\end{array}$ & Elevated & $\begin{array}{c}\text { Range } \\
\text { (elevated) }\end{array}$ & Reduced & $\begin{array}{c}\text { Range } \\
\text { (reduced) }\end{array}$ & $\begin{array}{c}\text { P value } \\
\text { (reduced) }\end{array}$ & Elevated & $\begin{array}{c}\text { Range } \\
\text { (elevated) }\end{array}$ & $\begin{array}{l}\text { P value } \\
\text { (elevated) }\end{array}$ \\
\hline Cortisol & $0 / 3(0 \%)$ & N/A & $\begin{array}{c}1 / 3 \\
(33.3 \%)\end{array}$ & $24.79-24.79$ & $\begin{array}{c}58 / 508 \\
(11.4 \%)\end{array}$ & $0.61-6.1$ & 1 & $\begin{array}{l}51 / 508 \\
(10 \%)\end{array}$ & $12.79-40.8$ & 0.2758 \\
\hline $\begin{array}{c}\text { Parathyroid } \\
\text { Hormone }\end{array}$ & $0 / 2(0 \%)$ & N/A & $0 / 2(0 \%)$ & N/A & $\begin{array}{l}11 / 448 \\
(2.5 \%)\end{array}$ & $10.5-14.3$ & 1 & $\begin{array}{c}6 / 448 \\
(1.3 \%)\end{array}$ & $66.33-80.19$ & 1 \\
\hline Estradiol & $0 / 2(0 \%)$ & N/A & $0 / 2(0 \%)$ & N/A & $\begin{array}{l}359 / 489 \\
(73.4 \%)\end{array}$ & $5.25-25.7$ & 0.0719 & $0 / 489(0 \%)$ & N/A & 1 \\
\hline FSH & $0 / 2(0 \%)$ & N/A & $0 / 2(0 \%)$ & N/A & $\begin{array}{l}38 / 456 \\
(8.3 \%)\end{array}$ & $0.117-1.43$ & 1 & $\begin{array}{l}2 / 456 \\
(0.4 \%)\end{array}$ & $14.24-20.3$ & 1 \\
\hline LH & $0 / 2(0 \%)$ & N/A & $\begin{array}{c}1 / 2 \\
(50 \%)\end{array}$ & $10-10$ & $\begin{array}{l}18 / 459 \\
(3.9 \%)\end{array}$ & $0.116-1.6$ & 1 & $\begin{array}{l}24 / 459 \\
(5.2 \%)\end{array}$ & $8.69-13.53$ & 0.1056 \\
\hline Progesterone & $0 / 2(0 \%)$ & N/A & $\begin{array}{c}1 / 2 \\
(50 \%)\end{array}$ & $0.19-0.19$ & $\begin{array}{c}56 / 454 \\
(12.3 \%)\end{array}$ & $0.058-0.194$ & 1 & $\begin{array}{l}35 / 454 \\
(7.7 \%)\end{array}$ & $0.15-1.69$ & 0.1518 \\
\hline $\begin{array}{c}\text { Total } \\
\text { Testosterone }\end{array}$ & $\begin{array}{c}1 / 3 \\
(33.3 \%)\end{array}$ & N/A & $0 / 3(0 \%)$ & N/A & $\begin{array}{c}60 / 533 \\
(11.3 \%)\end{array}$ & $10-344.3$ & 0.3045 & $\begin{array}{l}24 / 533 \\
(4.5 \%)\end{array}$ & $837.7-1210$ & 1 \\
\hline SHBG & $0 / 3(0 \%)$ & N/A & $0 / 3(0 \%)$ & N/A & $\begin{array}{l}37 / 510 \\
(7.3 \%)\end{array}$ & $6.4-16.4$ & 1 & $\begin{array}{c}92 / 510 \\
(18 \%)\end{array}$ & $56.04-226.6$ & 1 \\
\hline DHEA-S & $0 / 3(0 \%)$ & N/A & $0 / 3(0 \%)$ & N/A & $\begin{array}{l}21 / 505 \\
(4.2 \%)\end{array}$ & $34.6-203.5$ & 1 & $\begin{array}{c}74 / 505 \\
(14.7 \%)\end{array}$ & $261.6-955.3$ & 1 \\
\hline IGF-I & $\begin{array}{c}1 / 1 \\
(100 \%)\end{array}$ & $110-110$ & $0 / 1(0 \%)$ & N/A & $\begin{array}{l}8 / 240 \\
(3.3 \%)\end{array}$ & $56.23-97.6$ & 0.0373 & $\begin{array}{c}42 / 240 \\
(17.5 \%)\end{array}$ & $303.55-599$ & 1 \\
\hline Estriol & $0 / 1(0 \%)$ & N/A & $0 / 1(0 \%)$ & N/A & $0 / 70(0 \%)$ & N/A & 1 & $1 / 70(1.4 \%)$ & $210.5-210.5$ & 1 \\
\hline Estrone & $0 / 1(0 \%)$ & N/A & $0 / 1(0 \%)$ & N/A & $\begin{array}{c}22 / 79 \\
(27.8 \%)\end{array}$ & $9-10$ & 1 & $0 / 79(0 \%)$ & N/A & 1 \\
\hline Prolactin & N/A & N/A & N/A & N/A & $1 / 15(6.7 \%)$ & $3.69-3.69$ & 1 & $\begin{array}{c}4 / 15 \\
(26.7 \%)\end{array}$ & $15.26-21.96$ & 1 \\
\hline
\end{tabular}

Table S23. Age group 15 - 25. Reproductive hormonal changes in men with positive anti-TPO. The significance was not considered when the total number of subjects tested for each hormonal group is $\leq 10$.

\begin{tabular}{|c|c|c|c|c|c|c|c|c|c|c|}
\hline & \multicolumn{4}{|c|}{ Anti-TPO+ $(\mathrm{n}=447)$} & \multicolumn{6}{|c|}{ Anti-TPO- $(\mathrm{n}=184)$} \\
\hline & Reduced & $\begin{array}{c}\text { Range } \\
\text { (reduced) }\end{array}$ & Elevated & $\begin{array}{c}\text { Range } \\
\text { (elevated) }\end{array}$ & Reduced & $\begin{array}{c}\text { Range } \\
\text { (reduced) }\end{array}$ & $\begin{array}{c}\text { P value } \\
\text { (reduced) }\end{array}$ & Elevated & $\begin{array}{c}\text { Range } \\
\text { (elevated) }\end{array}$ & $\begin{array}{l}\text { P value } \\
\text { (elevated) }\end{array}$ \\
\hline Cortisol & $\begin{array}{l}45 / 400 \\
(11.3 \%)\end{array}$ & $0.61-6.1$ & $\begin{array}{l}35 / 400 \\
(8.8 \%)\end{array}$ & $\begin{array}{c}12.79- \\
30.97\end{array}$ & $\begin{array}{c}21 / 172 \\
(12.2 \%)\end{array}$ & $1.94-5.99$ & 0.852 & $\begin{array}{c}22 / 172 \\
(12.8 \%)\end{array}$ & $12.8-40.8$ & 0.1844 \\
\hline $\begin{array}{l}\text { Parathyroid } \\
\text { Hormone }\end{array}$ & $\begin{array}{l}9 / 342 \\
(2.6 \%)\end{array}$ & $10.5-14.16$ & $\begin{array}{l}5 / 342 \\
(1.5 \%)\end{array}$ & $\begin{array}{c}66.33- \\
80.19\end{array}$ & $\begin{array}{l}5 / 157 \\
(3.2 \%)\end{array}$ & $8.8-14.3$ & 0.9557 & $\begin{array}{l}2 / 157 \\
(1.3 \%)\end{array}$ & $75.11-75.2$ & 1 \\
\hline Estradiol & $\begin{array}{l}271 / 380 \\
(71.3 \%)\end{array}$ & $5.25-25.7$ & $\begin{array}{c}0 / 380 \\
(0 \%)\end{array}$ & N/A & $\begin{array}{c}127 / 167 \\
(76 \%)\end{array}$ & $5.3-25.5$ & 0.2981 & $0 / 167(0 \%)$ & N/A & 1 \\
\hline FSH & $\begin{array}{l}32 / 347 \\
(9.2 \%)\end{array}$ & $0.117-1.44$ & $\begin{array}{c}0 / 347 \\
(0 \%)\end{array}$ & N/A & $\begin{array}{l}11 / 159 \\
(6.9 \%)\end{array}$ & $0.179-1.4$ & 0.4896 & $\begin{array}{l}2 / 159 \\
(1.3 \%)\end{array}$ & $14.24-20.3$ & 0.0983 \\
\hline $\mathrm{LH}$ & $\begin{array}{l}11 / 351 \\
(3.1 \%)\end{array}$ & $0.1-1.4$ & $\begin{array}{c}21 / 351 \\
(6 \%)\end{array}$ & $8.69-13.53$ & $\begin{array}{l}10 / 159 \\
(6.3 \%)\end{array}$ & $0.116-1.6$ & 0.1554 & $\begin{array}{l}6 / 159 \\
(3.8 \%)\end{array}$ & $9.4-10.1$ & 0.413 \\
\hline
\end{tabular}




\section{Continued}

\begin{tabular}{|c|c|c|c|c|c|c|c|c|c|c|}
\hline Progesterone & $\begin{array}{l}49 / 351 \\
(14 \%)\end{array}$ & $0.058-0.194$ & $\begin{array}{c}37 / 351 \\
(10.5 \%)\end{array}$ & $0.15-1.85$ & $\begin{array}{l}15 / 155 \\
(9.7 \%)\end{array}$ & $\begin{array}{c}0.066- \\
0.192\end{array}$ & 0.2337 & $\begin{array}{l}6 / 155 \\
(3.9 \%)\end{array}$ & $0.183-1.69$ & 0.021 \\
\hline $\begin{array}{c}\text { Total } \\
\text { Testosterone }\end{array}$ & $\begin{array}{c}43 / 421 \\
(10.2 \%)\end{array}$ & $10.3-332.2$ & $\begin{array}{c}21 / 421 \\
(5 \%)\end{array}$ & $837.7-1210$ & $\begin{array}{c}29 / 179 \\
(16.2 \%)\end{array}$ & $10-344.3$ & 0.0539 & $\begin{array}{l}6 / 179 \\
(3.4 \%)\end{array}$ & $851.7-1069$ & 0.5033 \\
\hline SHBG & $\begin{array}{l}34 / 395 \\
(8.6 \%)\end{array}$ & $5.57-16.4$ & $\begin{array}{c}65 / 395 \\
(16.5 \%)\end{array}$ & $56.04-179.8$ & $\begin{array}{l}10 / 175 \\
(5.7 \%)\end{array}$ & $9.9-14.88$ & 0.306 & $\begin{array}{c}31 / 175 \\
(17.7 \%)\end{array}$ & $\begin{array}{c}56.25- \\
226.6\end{array}$ & 0.8033 \\
\hline DHEA-S & $\begin{array}{l}21 / 393 \\
(5.3 \%)\end{array}$ & $34.6-203.5$ & $\begin{array}{c}53 / 393 \\
(13.5 \%)\end{array}$ & $304.2-955.3$ & $\begin{array}{l}6 / 173 \\
(3.5 \%)\end{array}$ & $43.4-186.8$ & 0.4531 & $\begin{array}{c}30 / 173 \\
(17.3 \%)\end{array}$ & $\begin{array}{c}261.6- \\
763.4\end{array}$ & 0.2867 \\
\hline IGF-I & $\begin{array}{l}11 / 177 \\
(6.2 \%)\end{array}$ & $39.7-145$ & $\begin{array}{c}24 / 177 \\
(13.6 \%)\end{array}$ & $303.55-599$ & $\begin{array}{c}3 / 91 \\
(3.3 \%)\end{array}$ & $56.23-97.6$ & 0.3944 & $\begin{array}{c}23 / 91 \\
(25.3 \%)\end{array}$ & $317.29-597$ & 0.0265 \\
\hline Estriol & $0 / 61(0 \%)$ & N/A & $\begin{array}{c}1 / 61 \\
(1.6 \%)\end{array}$ & $210.5-210.5$ & $0 / 14(0 \%)$ & N/A & 1 & $0 / 14(0 \%)$ & N/A & 1 \\
\hline Estrone & $\begin{array}{c}15 / 71 \\
(21.1 \%)\end{array}$ & $9-9.3$ & $0 / 71(0 \%)$ & N/A & $\begin{array}{c}8 / 14 \\
(57.1 \%)\end{array}$ & $10-$ Oct & 0.0146 & 0/14 (0\%) & N/A & 1 \\
\hline Prolactin & $1 / 12(8.3 \%)$ & $3.69-3.69$ & $\begin{array}{c}3 / 12 \\
(25 \%)\end{array}$ & $15.26-19.61$ & $0 / 4(0 \%)$ & N/A & 1 & $1 / 4(25 \%)$ & $\begin{array}{c}21.96- \\
21.96\end{array}$ & 1 \\
\hline
\end{tabular}

Table S24. Age group 15 - 25. Reproductive hormonal changes in men with positive anti-Tg. The significance was not considered when the total number of subjects tested for each hormonal group is $\leq 10$.

\begin{tabular}{|c|c|c|c|c|c|c|c|c|c|c|}
\hline & \multicolumn{4}{|c|}{ Anti-Tg+ $(n=350)$} & \multicolumn{6}{|c|}{ Anti-Tg- $(\mathrm{n}=281)$} \\
\hline & Reduced & $\begin{array}{c}\text { Range } \\
\text { (reduced) }\end{array}$ & Elevated & $\begin{array}{c}\text { Range } \\
\text { (elevated) }\end{array}$ & Reduced & $\begin{array}{c}\text { Range } \\
\text { (reduced) }\end{array}$ & $\begin{array}{l}\text { P value } \\
\text { (reduced) }\end{array}$ & Elevated & $\begin{array}{c}\text { Range } \\
\text { (elevated) }\end{array}$ & $\begin{array}{c}\text { P value } \\
\text { (elevated) }\end{array}$ \\
\hline Cortisol & $\begin{array}{c}34 / 314 \\
(10.8 \%)\end{array}$ & $1.8-6.06$ & $\begin{array}{l}27 / 314 \\
(8.6 \%)\end{array}$ & $12.79-40.8$ & $\begin{array}{c}32 / 258 \\
(12.4 \%)\end{array}$ & $0.61-6.1$ & 0.649 & $\begin{array}{c}30 / 258 \\
(11.6 \%)\end{array}$ & $13.38-32.5$ & 0.2877 \\
\hline $\begin{array}{c}\text { Parathyroid } \\
\text { Hormone }\end{array}$ & $\begin{array}{l}11 / 271 \\
(4.1 \%)\end{array}$ & $8.8-14.16$ & $\begin{array}{c}3 / 271 \\
(1.1 \%)\end{array}$ & $\begin{array}{c}66.33- \\
80.19\end{array}$ & $\begin{array}{c}3 / 228 \\
(1.3 \%)\end{array}$ & $13.94-14.3$ & 0.0999 & $\begin{array}{c}4 / 228 \\
(1.8 \%)\end{array}$ & $71.31-75.2$ & 0.7076 \\
\hline Estradiol & $\begin{array}{l}220 / 304 \\
(72.4 \%)\end{array}$ & $5.5-25.63$ & $0 / 304(0 \%)$ & $\mathrm{N} / \mathrm{A}$ & $\begin{array}{l}178 / 243 \\
(73.3 \%)\end{array}$ & $5.25-25.7$ & 0.8936 & $0 / 243(0 \%)$ & N/A & 1 \\
\hline $\mathrm{FSH}$ & $\begin{array}{l}23 / 278 \\
(8.3 \%)\end{array}$ & $0.117-1.43$ & $\begin{array}{c}1 / 278 \\
(0.4 \%)\end{array}$ & $\begin{array}{c}14.24- \\
14.24\end{array}$ & $\begin{array}{l}20 / 228 \\
(8.8 \%)\end{array}$ & $0.179-1.44$ & 0.9682 & $\begin{array}{c}1 / 228 \\
(0.4 \%)\end{array}$ & $20.3-20.3$ & 1 \\
\hline $\mathrm{LH}$ & $\begin{array}{l}13 / 282 \\
(4.6 \%)\end{array}$ & $0.1-1.6$ & $\begin{array}{c}14 / 282 \\
(5 \%)\end{array}$ & $8.69-13.53$ & $\begin{array}{c}8 / 228 \\
(3.5 \%)\end{array}$ & $0.116-1.4$ & 0.6905 & $\begin{array}{l}13 / 228 \\
(5.7 \%)\end{array}$ & $9.02-12.85$ & 0.8644 \\
\hline Progesterone & $\begin{array}{c}28 / 276 \\
(10.1 \%)\end{array}$ & $\begin{array}{c}0.059- \\
0.193\end{array}$ & $\begin{array}{c}29 / 276 \\
(10.5 \%)\end{array}$ & $0.15-1.85$ & $\begin{array}{c}36 / 230 \\
(15.7 \%)\end{array}$ & $\begin{array}{c}0.058- \\
0.194\end{array}$ & 0.0852 & $\begin{array}{l}14 / 230 \\
(6.1 \%)\end{array}$ & $0.15-0.588$ & 0.1062 \\
\hline $\begin{array}{c}\text { Total } \\
\text { Testosterone }\end{array}$ & $\begin{array}{l}40 / 334 \\
(12 \%)\end{array}$ & $\begin{array}{c}21.82- \\
344.3\end{array}$ & $\begin{array}{l}15 / 334 \\
(4.5 \%)\end{array}$ & $848.5-1210$ & $\begin{array}{c}32 / 266 \\
(12 \%)\end{array}$ & $10-332.2$ & 1 & $\begin{array}{l}12 / 266 \\
(4.5 \%)\end{array}$ & $837.7-1069$ & 1 \\
\hline SHBG & $\begin{array}{l}25 / 316 \\
(7.9 \%)\end{array}$ & $5.57-16.22$ & $\begin{array}{c}47 / 316 \\
(14.9 \%)\end{array}$ & $\begin{array}{c}56.04- \\
226.6\end{array}$ & $\begin{array}{l}19 / 254 \\
(7.5 \%)\end{array}$ & $6.4-16.4$ & 0.973 & $\begin{array}{c}49 / 254 \\
(19.3 \%)\end{array}$ & $\begin{array}{c}56.25- \\
179.8\end{array}$ & 0.1977 \\
\hline DHEA-S & $\begin{array}{l}17 / 315 \\
(5.4 \%)\end{array}$ & $34.6-186.2$ & $\begin{array}{l}44 / 315 \\
(14 \%)\end{array}$ & $\begin{array}{c}369.5- \\
955.3\end{array}$ & $\begin{array}{c}10 / 251 \\
(4 \%)\end{array}$ & $43.4-203.5$ & 0.5586 & $\begin{array}{c}39 / 251 \\
(15.5 \%)\end{array}$ & $\begin{array}{c}261.6- \\
845.4\end{array}$ & 0.6856 \\
\hline IGF-I & $\begin{array}{c}8 / 138 \\
(5.8 \%)\end{array}$ & $39.7-110$ & $\begin{array}{c}21 / 138 \\
(15.2 \%)\end{array}$ & $303.55-599$ & $\begin{array}{c}6 / 130 \\
(4.6 \%)\end{array}$ & $79.9-145$ & 0.873 & $\begin{array}{c}26 / 130 \\
(20 \%)\end{array}$ & $323.92-597$ & 0.3853 \\
\hline Estriol & $0 / 43(0 \%)$ & $\mathrm{N} / \mathrm{A}$ & $0 / 43(0 \%)$ & $\mathrm{N} / \mathrm{A}$ & $0 / 32(0 \%)$ & $\mathrm{N} / \mathrm{A}$ & 1 & $\begin{array}{c}1 / 32 \\
(3.1 \%)\end{array}$ & $\begin{array}{c}210.5- \\
210.5\end{array}$ & 0.4267 \\
\hline Prolactin & $1 / 5(20 \%)$ & $3.69-3.69$ & $1 / 5(20 \%)$ & $\begin{array}{c}19.61- \\
19.61\end{array}$ & $0 / 11(0 \%)$ & $\mathrm{N} / \mathrm{A}$ & 0.3125 & $\begin{array}{c}3 / 11 \\
(27.3 \%)\end{array}$ & $\begin{array}{c}15.26- \\
21.96\end{array}$ & 1 \\
\hline
\end{tabular}


Table S25. Age group 26 - 35. Reproductive hormonal changes in men with hypothyroidism. The significance was not considered when the total number of subjects tested for each hormonal group is $\leq 10$.

\begin{tabular}{|c|c|c|c|c|c|c|c|c|c|c|}
\hline & \multicolumn{4}{|c|}{ Hypothyroidism $(\mathrm{n}=62)$} & \multicolumn{6}{|c|}{ Thyroid Negative $(\mathrm{n}=1090)$} \\
\hline & Reduced & $\begin{array}{c}\text { Range } \\
\text { (reduced) }\end{array}$ & Elevated & $\begin{array}{c}\text { Range } \\
\text { (elevated) }\end{array}$ & Reduced & $\begin{array}{c}\text { Range } \\
\text { (reduced) }\end{array}$ & $\begin{array}{l}\text { P value } \\
\text { (reduced) }\end{array}$ & Elevated & $\begin{array}{c}\text { Range } \\
\text { (elevated) }\end{array}$ & $\begin{array}{l}\text { P value } \\
\text { (elevated) }\end{array}$ \\
\hline Cortisol & $\begin{array}{c}6 / 52 \\
(11.5 \%)\end{array}$ & $2.9-6.09$ & $\begin{array}{c}7 / 52 \\
(13.5 \%)\end{array}$ & $19.8-31.6$ & $\begin{array}{c}99 / 986 \\
(10 \%)\end{array}$ & $1.26-6.12$ & 0.9099 & $\begin{array}{l}83 / 986 \\
(8.4 \%)\end{array}$ & $11.98-31$ & 0.314 \\
\hline $\begin{array}{c}\text { Parathyroid } \\
\text { Hormone }\end{array}$ & $0 / 46(0 \%)$ & N/A & $\begin{array}{c}2 / 46 \\
(4.3 \%)\end{array}$ & $\begin{array}{c}66.37- \\
200.5\end{array}$ & $\begin{array}{l}12 / 847 \\
(1.4 \%)\end{array}$ & $8.36-14.29$ & 1 & $\begin{array}{l}23 / 847 \\
(2.7 \%)\end{array}$ & $\begin{array}{c}65.66- \\
115.2\end{array}$ & 0.3727 \\
\hline Estradiol & $\begin{array}{c}43 / 56 \\
(76.8 \%)\end{array}$ & $7.6-25.33$ & $\begin{array}{c}1 / 56 \\
(1.8 \%)\end{array}$ & $61.05-183$ & $\begin{array}{l}668 / 980 \\
(68.2 \%)\end{array}$ & $5.3-25.74$ & 0.2284 & $\begin{array}{l}12 / 980 \\
(1.2 \%)\end{array}$ & $65.8-189.8$ & 0.5165 \\
\hline FSH & $\begin{array}{c}6 / 50 \\
(12 \%)\end{array}$ & $1.13-1.42$ & $2 / 50(4 \%)$ & $\begin{array}{c}12.85- \\
48.77\end{array}$ & $\begin{array}{l}90 / 922 \\
(9.8 \%)\end{array}$ & $0.1-1.43$ & 0.7846 & $\begin{array}{l}13 / 922 \\
(1.4 \%)\end{array}$ & $\begin{array}{c}12.47- \\
40.77\end{array}$ & 0.178 \\
\hline $\mathrm{LH}$ & $\begin{array}{c}5 / 50 \\
(10 \%)\end{array}$ & $0.878-1.59$ & $\begin{array}{c}5 / 50 \\
(10 \%)\end{array}$ & $8.9-36.21$ & $\begin{array}{l}66 / 930 \\
(7.1 \%)\end{array}$ & $0.1-1.63$ & 0.6231 & $\begin{array}{l}50 / 930 \\
(5.4 \%)\end{array}$ & $8.78-34.71$ & 0.2853 \\
\hline Progesterone & $\begin{array}{c}16 / 49 \\
(32.7 \%)\end{array}$ & $\begin{array}{c}0.063- \\
0.191\end{array}$ & $\begin{array}{c}2 / 49 \\
(4.1 \%)\end{array}$ & $0.156-6.98$ & $\begin{array}{l}192 / 869 \\
(22.1 \%)\end{array}$ & $\begin{array}{c}0.052- \\
0.194\end{array}$ & 0.123 & $\begin{array}{l}45 / 869 \\
(5.2 \%)\end{array}$ & $\begin{array}{c}0.151- \\
17.43\end{array}$ & 1 \\
\hline $\begin{array}{c}\text { Total } \\
\text { Testosterone }\end{array}$ & $\begin{array}{c}7 / 62 \\
(11.3 \%)\end{array}$ & $\begin{array}{c}16.75- \\
242.6\end{array}$ & $\begin{array}{c}3 / 62 \\
(4.8 \%)\end{array}$ & $772.6-1425$ & $\begin{array}{c}150 / 1066 \\
(14.1 \%)\end{array}$ & $\begin{array}{c}11.44- \\
345.3\end{array}$ & 0.6699 & $\begin{array}{c}57 / 1066 \\
(5.3 \%)\end{array}$ & $838.4-1437$ & 1 \\
\hline SHBG & $\begin{array}{c}5 / 59 \\
(8.5 \%)\end{array}$ & $10.9-16.3$ & $\begin{array}{c}10 / 59 \\
(16.9 \%)\end{array}$ & $58.2-167.9$ & $\begin{array}{c}72 / 1035 \\
(7 \%)\end{array}$ & $3.6-16.31$ & 0.8558 & $\begin{array}{c}151 / 1035 \\
(14.6 \%)\end{array}$ & $56-186.7$ & 0.7575 \\
\hline DHEA-S & $\begin{array}{c}4 / 56 \\
(7.1 \%)\end{array}$ & $21.94-157$ & $\begin{array}{c}7 / 56 \\
(12.5 \%)\end{array}$ & $296-855.9$ & 69/983 (7\%) & $37.6-205.6$ & 1 & $\begin{array}{l}173 / 983 \\
(17.6 \%)\end{array}$ & $\begin{array}{c}436.8- \\
922.3\end{array}$ & 0.4242 \\
\hline IGF-I & $1 / 20(5 \%)$ & $\begin{array}{c}30.41- \\
30.41\end{array}$ & $\begin{array}{c}2 / 20 \\
(10 \%)\end{array}$ & $283-319.03$ & $\begin{array}{l}14 / 477 \\
(2.9 \%)\end{array}$ & $\begin{array}{c}18.09- \\
96.15\end{array}$ & 0.4648 & $\begin{array}{l}21 / 477 \\
(4.4 \%)\end{array}$ & $241-487.33$ & 0.2352 \\
\hline Estriol & $0 / 6(0 \%)$ & N/A & $0 / 6(0 \%)$ & N/A & $0 / 113(0 \%)$ & N/A & 1 & $2 / 113(1.8 \%)$ & $344-426.5$ & 1 \\
\hline Estrone & $0 / 6(0 \%)$ & N/A & $0 / 6(0 \%)$ & N/A & $\begin{array}{c}28 / 150 \\
(18.7 \%)\end{array}$ & $9.4-9.9$ & 0.5921 & $0 / 150(0 \%)$ & N/A & 1 \\
\hline Prolactin & $0 / 2(0 \%)$ & N/A & $0 / 2(0 \%)$ & N/A & $0 / 39(0 \%)$ & N/A & 1 & $9 / 39(23.1 \%)$ & $\begin{array}{c}15.65- \\
33.95\end{array}$ & 1 \\
\hline
\end{tabular}

Table S26. Age group 26 - 35. Reproductive hormonal changes in men with hyperthyroidism. The significance was not considered when the total number of subjects tested for each hormonal group is $\leq 10$.

\begin{tabular}{|c|c|c|c|c|c|c|c|c|c|c|}
\hline & \multicolumn{4}{|c|}{ Hyperthyroidism $(\mathrm{n}=7)$} & \multicolumn{6}{|c|}{ Thyroid Negative $(\mathrm{n}=1090)$} \\
\hline & Reduced & $\begin{array}{c}\text { Range } \\
\text { (reduced) }\end{array}$ & Elevated & $\begin{array}{c}\text { Range } \\
\text { (elevated) }\end{array}$ & Reduced & $\begin{array}{c}\text { Range } \\
\text { (reduced) }\end{array}$ & $\begin{array}{c}\text { P value } \\
\text { (reduced) }\end{array}$ & Elevated & $\begin{array}{c}\text { Range } \\
\text { (elevated) }\end{array}$ & $\begin{array}{l}\text { P value } \\
\text { (elevated) }\end{array}$ \\
\hline Cortisol & $\begin{array}{c}1 / 5 \\
(20 \%)\end{array}$ & $4.3-4.3$ & $0 / 5(0 \%)$ & $\mathrm{N} / \mathrm{A}$ & $\begin{array}{c}99 / 986 \\
(10 \%)\end{array}$ & $1.26-6.12$ & 0.4132 & $\begin{array}{l}83 / 986 \\
(8.4 \%)\end{array}$ & $11.98-31$ & 1 \\
\hline $\begin{array}{l}\text { Parathyroid } \\
\text { Hormone }\end{array}$ & $0 / 4(0 \%)$ & N/A & $0 / 4(0 \%)$ & N/A & $\begin{array}{l}12 / 847 \\
(1.4 \%)\end{array}$ & $8.36-14.29$ & 1 & $\begin{array}{l}23 / 847 \\
(2.7 \%)\end{array}$ & $65.66-115.2$ & 1 \\
\hline Estradiol & $\begin{array}{c}3 / 7 \\
(42.9 \%)\end{array}$ & $9.6-21.1$ & $0 / 7(0 \%)$ & N/A & $\begin{array}{l}668 / 980 \\
(68.2 \%)\end{array}$ & $5.3-25.74$ & 0.2193 & $\begin{array}{l}12 / 980 \\
(1.2 \%)\end{array}$ & $65.8-189.8$ & 1 \\
\hline FSH & $0 / 6(0 \%)$ & N/A & $\begin{array}{c}2 / 6 \\
(33.3 \%)\end{array}$ & $13.8-52.8$ & $\begin{array}{l}90 / 922 \\
(9.8 \%)\end{array}$ & $0.1-1.43$ & 1 & $\begin{array}{l}13 / 922 \\
(1.4 \%)\end{array}$ & $12.47-40.77$ & 0.0035 \\
\hline $\mathrm{LH}$ & $0 / 5(0 \%)$ & N/A & $\begin{array}{c}2 / 5 \\
(40 \%)\end{array}$ & $9.15-28.38$ & $\begin{array}{l}66 / 930 \\
(7.1 \%)\end{array}$ & $0.1-1.63$ & 1 & $\begin{array}{l}50 / 930 \\
(5.4 \%)\end{array}$ & $8.78-34.71$ & 0.0272 \\
\hline
\end{tabular}




\section{Continued}

\begin{tabular}{|c|c|c|c|c|c|c|c|c|c|c|}
\hline Progesterone & $0 / 4(0 \%)$ & N/A & $\begin{array}{c}1 / 4 \\
(25 \%)\end{array}$ & $0.184-0.184$ & $\begin{array}{l}192 / 869 \\
(22.1 \%)\end{array}$ & $\begin{array}{c}0.052- \\
0.194\end{array}$ & 0.5814 & $\begin{array}{l}45 / 869 \\
(5.2 \%)\end{array}$ & $0.151-17.43$ & 0.195 \\
\hline $\begin{array}{c}\text { Total } \\
\text { Testosterone }\end{array}$ & $\begin{array}{c}4 / 6 \\
(66.7 \%)\end{array}$ & $30.7-110.2$ & $\begin{array}{c}1 / 6 \\
(16.7 \%)\end{array}$ & $744.6-1433$ & $\begin{array}{c}150 / 1066 \\
(14.1 \%)\end{array}$ & $\begin{array}{c}11.44- \\
345.3\end{array}$ & 0.0049 & $\begin{array}{c}57 / 1066 \\
(5.3 \%)\end{array}$ & $838.4-1437$ & 0.2843 \\
\hline SHBG & $\begin{array}{c}1 / 6 \\
(16.7 \%)\end{array}$ & $9.04-9.04$ & $\begin{array}{c}1 / 6 \\
(16.7 \%)\end{array}$ & $63.9-179.6$ & $\begin{array}{c}72 / 1035 \\
(7 \%)\end{array}$ & $3.6-16.31$ & 0.3542 & $\begin{array}{c}151 / 1035 \\
(14.6 \%)\end{array}$ & $56-186.7$ & 1 \\
\hline DHEA-S & $\begin{array}{c}2 / 7 \\
(28.6 \%)\end{array}$ & $23.79-104.7$ & $\begin{array}{c}1 / 7 \\
(14.3 \%)\end{array}$ & $359.8-773.8$ & $69 / 983(7 \%)$ & $37.6-205.6$ & 0.0842 & $\begin{array}{l}173 / 983 \\
(17.6 \%)\end{array}$ & $436.8-922.3$ & 1 \\
\hline IGF-I & $0 / 4(0 \%)$ & N/A & $\begin{array}{c}1 / 4 \\
(25 \%)\end{array}$ & $\begin{array}{c}347.91- \\
347.91\end{array}$ & $\begin{array}{l}14 / 477 \\
(2.9 \%)\end{array}$ & $\begin{array}{c}18.09- \\
96.15\end{array}$ & 1 & $\begin{array}{l}21 / 477 \\
(4.4 \%)\end{array}$ & $241-487.33$ & 0.1713 \\
\hline Estriol & N/A & N/A & N/A & N/A & 0/113 (0\%) & N/A & 1 & $\begin{array}{c}2 / 113 \\
(1.8 \%)\end{array}$ & $344-426.5$ & 1 \\
\hline Estrone & 0/1 (0\%) & N/A & $0 / 1(0 \%)$ & N/A & $\begin{array}{c}28 / 150 \\
(18.7 \%)\end{array}$ & $9.4-9.9$ & 1 & $\begin{array}{c}0 / 150 \\
(0 \%)\end{array}$ & N/A & 1 \\
\hline Prolactin & $0 / 1(0 \%)$ & N/A & $\begin{array}{c}1 / 1 \\
(100 \%)\end{array}$ & 20.28 & $0 / 39(0 \%)$ & N/A & 1 & $\begin{array}{c}9 / 39 \\
(23.1 \%)\end{array}$ & $15.65-33.95$ & 0.25 \\
\hline
\end{tabular}

Table S27. Age group 26 - 35. Reproductive hormonal changes in men with positive anti-TPO. The significance was not considered when the total number of subjects tested for each hormonal group is $\leq 10$.

\begin{tabular}{|c|c|c|c|c|c|c|c|c|c|c|}
\hline & \multicolumn{4}{|c|}{ Anti-TPO+ $(\mathrm{n}=888)$} & \multicolumn{6}{|c|}{ Anti-TPO- $(n=293)$} \\
\hline & Reduced & $\begin{array}{c}\text { Range } \\
\text { (reduced) }\end{array}$ & Elevated & $\begin{array}{c}\text { Range } \\
\text { (elevated) }\end{array}$ & Reduced & $\begin{array}{c}\text { Range } \\
\text { (reduced) }\end{array}$ & $\begin{array}{c}\text { P value } \\
\text { (reduced) }\end{array}$ & Elevated & $\begin{array}{c}\text { Range } \\
\text { (elevated) }\end{array}$ & $\begin{array}{c}\text { P value } \\
\text { (elevated) }\end{array}$ \\
\hline Cortisol & $\begin{array}{c}85 / 794 \\
(10.7 \%)\end{array}$ & $1.26-6.09$ & $\begin{array}{l}51 / 794 \\
(6.4 \%)\end{array}$ & $12.06-31.6$ & $\begin{array}{l}22 / 264 \\
(8.3 \%)\end{array}$ & $1.64-6.12$ & 0.3224 & $\begin{array}{c}41 / 264 \\
(15.5 \%)\end{array}$ & $11.98-31$ & 0 \\
\hline $\begin{array}{c}\text { Parathyroid } \\
\text { Hormone }\end{array}$ & $\begin{array}{c}8 / 681 \\
(1.2 \%)\end{array}$ & $8.36-14.29$ & $\begin{array}{l}18 / 681 \\
(2.6 \%)\end{array}$ & $65.5-172$ & $\begin{array}{c}4 / 236 \\
(1.7 \%)\end{array}$ & $8.8-13.9$ & 0.5173 & $\begin{array}{c}8 / 236 \\
(3.4 \%)\end{array}$ & $66.7-81.73$ & 0.7129 \\
\hline Estradiol & $\begin{array}{l}545 / 808 \\
(67.5 \%)\end{array}$ & $5.3-25.74$ & $\begin{array}{l}12 / 808 \\
(1.5 \%)\end{array}$ & $61.03-313$ & $\begin{array}{l}182 / 257 \\
(70.8 \%)\end{array}$ & $5.8-25.61$ & 0.3508 & $\begin{array}{c}5 / 257 \\
(1.9 \%)\end{array}$ & $65.9-189.8$ & 0.8203 \\
\hline FSH & $\begin{array}{c}76 / 740 \\
(10.3 \%)\end{array}$ & $0.1-1.43$ & $\begin{array}{l}16 / 740 \\
(2.2 \%)\end{array}$ & $\begin{array}{c}12.48- \\
117.5\end{array}$ & $\begin{array}{c}27 / 252 \\
(10.7 \%)\end{array}$ & $0.15-1.4$ & 0.9362 & $\begin{array}{c}1 / 252 \\
(0.4 \%)\end{array}$ & $\begin{array}{c}13.58- \\
13.58\end{array}$ & 0.0878 \\
\hline $\mathrm{LH}$ & $\begin{array}{l}51 / 745 \\
(6.8 \%)\end{array}$ & $0.1-1.63$ & $\begin{array}{l}44 / 745 \\
(5.9 \%)\end{array}$ & $8.7-46.2$ & $\begin{array}{c}26 / 254 \\
(10.2 \%)\end{array}$ & $0.3-1.6$ & 0.1067 & $\begin{array}{l}14 / 254 \\
(5.5 \%)\end{array}$ & $8.78-16.5$ & 0.9389 \\
\hline Progesterone & $\begin{array}{l}172 / 699 \\
(24.6 \%)\end{array}$ & $\begin{array}{c}0.052- \\
0.194\end{array}$ & $\begin{array}{l}38 / 699 \\
(5.4 \%)\end{array}$ & $0.15-6.98$ & $\begin{array}{c}38 / 239 \\
(15.9 \%)\end{array}$ & $0.064-0.19$ & 0.007 & $\begin{array}{l}13 / 239 \\
(5.4 \%)\end{array}$ & $\begin{array}{c}0.191- \\
17.43\end{array}$ & 1 \\
\hline $\begin{array}{c}\text { Total } \\
\text { Testosterone }\end{array}$ & $\begin{array}{l}107 / 871 \\
(12.3 \%)\end{array}$ & $\begin{array}{c}11.44- \\
345.3\end{array}$ & $\begin{array}{l}49 / 871 \\
(5.6 \%)\end{array}$ & $375.5-1498$ & $\begin{array}{c}55 / 283 \\
(19.4 \%)\end{array}$ & $15.3-345$ & 0.0036 & $\begin{array}{l}14 / 283 \\
(4.9 \%)\end{array}$ & $858.9-1440$ & 0.7748 \\
\hline SHBG & $\begin{array}{l}52 / 847 \\
(6.1 \%)\end{array}$ & $3.6-16.31$ & $\begin{array}{l}130 / 847 \\
(15.3 \%)\end{array}$ & $56.5-180.2$ & $\begin{array}{c}30 / 281 \\
(10.7 \%)\end{array}$ & 16 - May & 0.0161 & $\begin{array}{c}37 / 281 \\
(13.2 \%)\end{array}$ & $56.1-125.1$ & 0.4265 \\
\hline DHEA-S & $\begin{array}{l}58 / 799 \\
(7.3 \%)\end{array}$ & $\begin{array}{c}21.94- \\
160.2\end{array}$ & $\begin{array}{c}136 / 799 \\
(17 \%)\end{array}$ & $\begin{array}{c}248.9- \\
971.8\end{array}$ & $\begin{array}{l}21 / 268 \\
(7.8 \%)\end{array}$ & $\begin{array}{c}59.05- \\
205.6\end{array}$ & 0.8593 & $\begin{array}{c}50 / 268 \\
(18.7 \%)\end{array}$ & $\begin{array}{c}453.6- \\
917.2\end{array}$ & 0.6047 \\
\hline IGF-I & $\begin{array}{c}11 / 367 \\
(3 \%)\end{array}$ & $\begin{array}{c}30.41- \\
96.15\end{array}$ & $\begin{array}{l}17 / 367 \\
(4.6 \%)\end{array}$ & $189-354.09$ & $\begin{array}{c}4 / 141 \\
(2.8 \%)\end{array}$ & 18.09 - 93.4 & 1 & $7 / 141(5 \%)$ & $302-487.33$ & 1 \\
\hline Estriol & $0 / 98(0 \%)$ & $\mathrm{N} / \mathrm{A}$ & $1 / 98(1 \%)$ & $\begin{array}{c}227.9- \\
664.4\end{array}$ & $0 / 25(0 \%)$ & N/A & 1 & $1 / 25(4 \%)$ & $344-344$ & 0.3665 \\
\hline Prolactin & $0 / 37(0 \%)$ & $\mathrm{N} / \mathrm{A}$ & $\begin{array}{c}7 / 37 \\
(18.9 \%)\end{array}$ & $\begin{array}{c}15.49- \\
127.8\end{array}$ & $0 / 5(0 \%)$ & $\mathrm{N} / \mathrm{A}$ & 1 & $3 / 5(60 \%)$ & $\begin{array}{c}17.14- \\
33.95\end{array}$ & 0.0782 \\
\hline
\end{tabular}


Table S28. Age group 26 - 35. Reproductive hormonal changes in men with positive anti-Tg. The significance was not considered when the total number of subjects tested for each hormonal group is $\leq 10$.

\begin{tabular}{|c|c|c|c|c|c|c|c|c|c|c|}
\hline & \multicolumn{4}{|c|}{ Anti-Tg+ $(n=681)$} & \multicolumn{6}{|c|}{ Anti-Tg- $(\mathrm{n}=504)$} \\
\hline & Reduced & $\begin{array}{c}\text { Range } \\
\text { (reduced) }\end{array}$ & Elevated & $\begin{array}{c}\text { Range } \\
\text { (elevated) }\end{array}$ & Reduced & $\begin{array}{c}\text { Range } \\
\text { (reduced) }\end{array}$ & $\begin{array}{l}\text { P value } \\
\text { (reduced) }\end{array}$ & Elevated & $\begin{array}{c}\text { Range } \\
\text { (elevated) }\end{array}$ & $\begin{array}{l}\text { P value } \\
\text { (elevated) }\end{array}$ \\
\hline Cortisol & $\begin{array}{l}56 / 613 \\
(9.1 \%)\end{array}$ & $1.26-6.09$ & $\begin{array}{l}54 / 613 \\
(8.8 \%)\end{array}$ & $12.06-31.6$ & $\begin{array}{c}52 / 448 \\
(11.6 \%)\end{array}$ & $1.64-6.12$ & 0.2254 & $\begin{array}{l}38 / 448 \\
(8.5 \%)\end{array}$ & $15.73-27.9$ & 0.939 \\
\hline $\begin{array}{c}\text { Parathyroid } \\
\text { Hormone }\end{array}$ & $\begin{array}{c}8 / 524 \\
(1.5 \%)\end{array}$ & $8.36-14.29$ & $\begin{array}{l}15 / 524 \\
(2.9 \%)\end{array}$ & $65.5-174.4$ & $4 / 396(1 \%)$ & $8.8-13$ & 0.5693 & $\begin{array}{l}11 / 396 \\
(2.8 \%)\end{array}$ & $\begin{array}{c}65.66- \\
83.96\end{array}$ & 1 \\
\hline Estradiol & $\begin{array}{l}417 / 619 \\
(67.4 \%)\end{array}$ & $5.3-25.74$ & $\begin{array}{l}11 / 619 \\
(1.8 \%)\end{array}$ & $\begin{array}{c}61.03- \\
141.5\end{array}$ & $\begin{array}{l}312 / 448 \\
(69.6 \%)\end{array}$ & $5.58-25.68$ & 0.4702 & $\begin{array}{c}6 / 448 \\
(1.3 \%)\end{array}$ & $65.2-189.8$ & 0.752 \\
\hline FSH & $\begin{array}{c}63 / 574 \\
(11 \%)\end{array}$ & $0.1-1.43$ & $\begin{array}{l}12 / 574 \\
(2.1 \%)\end{array}$ & $\begin{array}{c}12.48- \\
117.5\end{array}$ & $\begin{array}{l}40 / 421 \\
(9.5 \%)\end{array}$ & $0.103-1.4$ & 0.5164 & $\begin{array}{c}5 / 421 \\
(1.2 \%)\end{array}$ & $\begin{array}{c}12.47- \\
24.08\end{array}$ & 0.4019 \\
\hline $\mathrm{LH}$ & $\begin{array}{l}42 / 574 \\
(7.3 \%)\end{array}$ & $0.1-1.59$ & $\begin{array}{l}37 / 574 \\
(6.4 \%)\end{array}$ & $8.7-44.6$ & $\begin{array}{l}35 / 428 \\
(8.2 \%)\end{array}$ & $0.3-1.63$ & 0.6995 & $\begin{array}{l}21 / 428 \\
(4.9 \%)\end{array}$ & $8.71-21.3$ & 0.3705 \\
\hline Progesterone & $\begin{array}{l}108 / 543 \\
(19.9 \%)\end{array}$ & $\begin{array}{c}0.052- \\
0.194\end{array}$ & $\begin{array}{c}27 / 543 \\
(5 \%)\end{array}$ & $0.15-6.98$ & $\begin{array}{l}102 / 397 \\
(25.7 \%)\end{array}$ & $\begin{array}{c}0.056- \\
0.193\end{array}$ & 0.0423 & $\begin{array}{c}24 / 397 \\
(6 \%)\end{array}$ & $\begin{array}{c}0.152- \\
17.43\end{array}$ & 0.5676 \\
\hline $\begin{array}{c}\text { Total } \\
\text { Testosterone }\end{array}$ & $\begin{array}{l}100 / 671 \\
(14.9 \%)\end{array}$ & $16.75-345$ & $\begin{array}{l}36 / 671 \\
(5.4 \%)\end{array}$ & $375.5-1498$ & $\begin{array}{c}63 / 487 \\
(12.9 \%)\end{array}$ & $\begin{array}{c}11.44- \\
345.3\end{array}$ & 0.3874 & $\begin{array}{l}27 / 487 \\
(5.5 \%)\end{array}$ & $838.4-1437$ & 0.9989 \\
\hline SHBG & $\begin{array}{l}44 / 658 \\
(6.7 \%)\end{array}$ & $3.6-16.31$ & $\begin{array}{c}99 / 658 \\
(15 \%)\end{array}$ & $\begin{array}{c}59.19- \\
180.2\end{array}$ & $\begin{array}{c}38 / 473 \\
(8 \%)\end{array}$ & $4.2-16.31$ & 0.456 & $\begin{array}{c}69 / 473 \\
(14.6 \%)\end{array}$ & $56-186.7$ & 0.8975 \\
\hline DHEA-S & $\begin{array}{l}46 / 618 \\
(7.4 \%)\end{array}$ & $\begin{array}{c}21.94- \\
192.8\end{array}$ & $\begin{array}{l}108 / 618 \\
(17.5 \%)\end{array}$ & $\begin{array}{c}248.9- \\
971.8\end{array}$ & $\begin{array}{l}33 / 452 \\
(7.3 \%)\end{array}$ & $37.6-205.6$ & 1 & $\begin{array}{l}79 / 452 \\
(17.5 \%)\end{array}$ & $\begin{array}{c}436.8- \\
922.3\end{array}$ & 1 \\
\hline IGF-I & $\begin{array}{l}7 / 309 \\
(2.3 \%)\end{array}$ & $\begin{array}{c}30.41- \\
96.15\end{array}$ & $\begin{array}{l}12 / 309 \\
(3.9 \%)\end{array}$ & $189-392.6$ & $8 / 201(4 \%)$ & $18.09-93.4$ & 0.3943 & $\begin{array}{c}12 / 201 \\
(6 \%)\end{array}$ & $241-487.33$ & 0.3824 \\
\hline Estriol & $0 / 69(0 \%)$ & N/A & $2 / 69(2.9 \%)$ & $\begin{array}{c}233.4- \\
664.4\end{array}$ & $0 / 54(0 \%)$ & N/A & 1 & $0 / 54(0 \%)$ & N/A & 0.5034 \\
\hline Estrone & $\begin{array}{c}19 / 91 \\
(20.9 \%)\end{array}$ & $9.6-9.8$ & $0 / 91(0 \%)$ & N/A & $\begin{array}{c}10 / 71 \\
(14.1 \%)\end{array}$ & $9.4-9.9$ & 0.3614 & $\begin{array}{c}1 / 71 \\
(1.4 \%)\end{array}$ & $68.7-68.7$ & 0.4383 \\
\hline Prolactin & $0 / 23(0 \%)$ & N/A & $\begin{array}{c}6 / 23 \\
(26.1 \%)\end{array}$ & $\begin{array}{c}15.49- \\
127.8\end{array}$ & $0 / 19(0 \%)$ & N/A & 1 & $\begin{array}{c}4 / 19 \\
(21.1 \%)\end{array}$ & $\begin{array}{c}18.94- \\
33.95\end{array}$ & 1 \\
\hline
\end{tabular}

Table S29. Age group 36 - 49. Reproductive hormonal changes in men with hypothyroidism. The significance was not considered when the total number of subjects tested for each hormonal group is $\leq 10$.

\begin{tabular}{|c|c|c|c|c|c|c|c|c|c|c|}
\hline & \multicolumn{4}{|c|}{ Hypothyroidism $(\mathrm{n}=146)$} & \multicolumn{6}{|c|}{ Thyroid Negative $(\mathrm{n}=2333)$} \\
\hline & Reduced & $\begin{array}{c}\text { Range } \\
\text { (reduced) }\end{array}$ & Elevated & $\begin{array}{c}\text { Range } \\
\text { (elevated) }\end{array}$ & Reduced & $\begin{array}{c}\text { Range } \\
\text { (reduced) }\end{array}$ & $\begin{array}{l}\text { P value } \\
\text { (reduced) }\end{array}$ & Elevated & $\begin{array}{c}\text { Range } \\
\text { (elevated) }\end{array}$ & $\begin{array}{l}\text { P value } \\
\text { (elevated) }\end{array}$ \\
\hline Cortisol & $\begin{array}{l}14 / 127 \\
(11 \%)\end{array}$ & $1.06-6.07$ & $\begin{array}{l}9 / 127 \\
(7.1 \%)\end{array}$ & $15-27.7$ & $\begin{array}{c}233 / 2010 \\
(11.6 \%)\end{array}$ & $0.652-6.13$ & 0.9591 & $\begin{array}{c}111 / 2010 \\
(5.5 \%)\end{array}$ & $12.2-35.2$ & 0.5865 \\
\hline $\begin{array}{c}\text { Parathyroid } \\
\text { Hormone }\end{array}$ & $\begin{array}{l}1 / 110 \\
(0.9 \%)\end{array}$ & $11.6-11.6$ & $\begin{array}{l}4 / 110 \\
(3.6 \%)\end{array}$ & $\begin{array}{c}67.05- \\
101.5\end{array}$ & $\begin{array}{c}14 / 1709 \\
(0.8 \%)\end{array}$ & $9-14$ & 0.6091 & $\begin{array}{c}84 / 1709 \\
(4.9 \%)\end{array}$ & $\begin{array}{c}65.64- \\
465.4\end{array}$ & 0.8171 \\
\hline Estradiol & $\begin{array}{c}84 / 128 \\
(65.6 \%)\end{array}$ & $5.2-25.52$ & $\begin{array}{c}6 / 128 \\
(4.7 \%)\end{array}$ & $62.4-90.1$ & $\begin{array}{c}1365 / 2071 \\
(65.9 \%)\end{array}$ & $5.02-25.74$ & 1 & $\begin{array}{c}60 / 2071 \\
(2.9 \%)\end{array}$ & $\begin{array}{c}61.22- \\
247.4\end{array}$ & 0.3761 \\
\hline FSH & $\begin{array}{c}21 / 121 \\
(17.4 \%)\end{array}$ & $0.1-0.765$ & $\begin{array}{l}4 / 121 \\
(3.3 \%)\end{array}$ & $15.97-23$ & $\begin{array}{c}323 / 1913 \\
(16.9 \%)\end{array}$ & $0.1-1.44$ & 0.9928 & $\begin{array}{c}43 / 1913 \\
(2.2 \%)\end{array}$ & $12.5-63.9$ & 0.3591 \\
\hline $\mathrm{LH}$ & $\begin{array}{l}27 / 121 \\
(22.3 \%)\end{array}$ & $0.1-1.58$ & $\begin{array}{l}7 / 121 \\
(5.8 \%)\end{array}$ & $8.75-12.6$ & $\begin{array}{c}341 / 1936 \\
(17.6 \%)\end{array}$ & $0.1-1.64$ & 0.2354 & $\begin{array}{c}112 / 1936 \\
(5.8 \%)\end{array}$ & $8.68-53.67$ & 1 \\
\hline
\end{tabular}




\section{Continued}

\begin{tabular}{|c|c|c|c|c|c|c|c|c|c|c|}
\hline Progesterone & $\begin{array}{c}36 / 114 \\
(31.6 \%)\end{array}$ & $0.059-0.19$ & $\begin{array}{l}4 / 114 \\
(3.5 \%)\end{array}$ & $0.214-3.01$ & $\begin{array}{c}494 / 1801 \\
(27.4 \%)\end{array}$ & $\begin{array}{c}0.052- \\
0.194\end{array}$ & 0.394 & $\begin{array}{c}66 / 1801 \\
(3.7 \%)\end{array}$ & $\begin{array}{c}0.152- \\
17.02\end{array}$ & 1 \\
\hline $\begin{array}{c}\text { Total } \\
\text { Testosterone }\end{array}$ & $\begin{array}{l}29 / 144 \\
(20.1 \%)\end{array}$ & $\begin{array}{c}85.81- \\
334.6\end{array}$ & $\begin{array}{c}15 / 144 \\
(10.4 \%)\end{array}$ & $876.6-1488$ & $\begin{array}{c}363 / 2286 \\
(15.9 \%)\end{array}$ & $5.5-347.3$ & 0.2183 & $\begin{array}{c}239 / 2286 \\
(10.5 \%)\end{array}$ & $839.6-1499$ & 1 \\
\hline SHBG & $\begin{array}{l}11 / 140 \\
(7.9 \%)\end{array}$ & $3.9-15.91$ & $\begin{array}{c}18 / 140 \\
(12.9 \%)\end{array}$ & $\begin{array}{c}56.24- \\
118.4\end{array}$ & $\begin{array}{c}138 / 2217 \\
(6.2 \%)\end{array}$ & $3.9-16.44$ & 0.5547 & $\begin{array}{c}364 / 2217 \\
(16.4 \%)\end{array}$ & $\begin{array}{c}56.05- \\
181.7\end{array}$ & 0.3218 \\
\hline DHEA-S & $\begin{array}{l}5 / 132 \\
(3.8 \%)\end{array}$ & $\begin{array}{c}35.68- \\
79.45\end{array}$ & $\begin{array}{l}8 / 132 \\
(6.1 \%)\end{array}$ & $\begin{array}{c}375.2- \\
545.5\end{array}$ & $\begin{array}{c}64 / 2084 \\
(3.1 \%)\end{array}$ & $8-159.3$ & 0.8403 & $\begin{array}{c}176 / 2084 \\
(8.4 \%)\end{array}$ & $331.6-972$ & 0.4236 \\
\hline IGF-I & $\begin{array}{c}4 / 58 \\
(6.9 \%)\end{array}$ & $\begin{array}{c}15.52- \\
90.15\end{array}$ & $\begin{array}{c}1 / 58 \\
(1.7 \%)\end{array}$ & $235-235$ & $\begin{array}{c}37 / 1042 \\
(3.6 \%)\end{array}$ & $\begin{array}{c}11.99- \\
99.36\end{array}$ & 0.2693 & $\begin{array}{c}42 / 1042 \\
(4 \%)\end{array}$ & $200-474.84$ & 0.7229 \\
\hline Estriol & $0 / 16(0 \%)$ & N/A & $\begin{array}{l}0 / 16 \\
(0 \%)\end{array}$ & N/A & $0 / 181(0 \%)$ & N/A & 1 & $\begin{array}{l}4 / 181 \\
(2.2 \%)\end{array}$ & $\begin{array}{l}208.4- \\
420.49\end{array}$ & 1 \\
\hline Estrone & $\begin{array}{c}3 / 27 \\
(11.1 \%)\end{array}$ & N/A & $\begin{array}{c}3 / 27 \\
(11.1 \%)\end{array}$ & $54.4-62.43$ & $\begin{array}{c}39 / 308 \\
(12.7 \%)\end{array}$ & $9.1-10.1$ & 1 & $\begin{array}{l}17 / 308 \\
(5.5 \%)\end{array}$ & $51.2-88.6$ & 0.2112 \\
\hline Prolactin & $0 / 9(0 \%)$ & N/A & $\begin{array}{c}4 / 9 \\
(44.4 \%)\end{array}$ & $\begin{array}{c}20.38- \\
24.09\end{array}$ & $3 / 97(3.1 \%)$ & $1.91-4.01$ & 1 & $\begin{array}{c}10 / 97 \\
(10.3 \%)\end{array}$ & $\begin{array}{c}15.69- \\
35.85\end{array}$ & 0.0168 \\
\hline
\end{tabular}

Table S30. Age group 36 - 49. Reproductive hormonal changes in men with hyperthyroidism. The significance was not considered when the total number of subjects tested for each hormonal group is $\leq 10$.

\begin{tabular}{|c|c|c|c|c|c|c|c|c|c|c|}
\hline & \multicolumn{4}{|c|}{ Hyperthyroidism $(\mathrm{n}=28)$} & \multicolumn{6}{|c|}{ Thyroid Negative $(\mathrm{n}=2333)$} \\
\hline & Reduced & $\begin{array}{c}\text { Range } \\
\text { (reduced) }\end{array}$ & Elevated & $\begin{array}{c}\text { Range } \\
\text { (elevated) }\end{array}$ & Reduced & $\begin{array}{c}\text { Range } \\
\text { (reduced) }\end{array}$ & $\begin{array}{l}\text { P value } \\
\text { (reduced) }\end{array}$ & Elevated & $\begin{array}{c}\text { Range } \\
\text { (elevated) }\end{array}$ & $\begin{array}{l}\text { P value } \\
\text { (elevated) }\end{array}$ \\
\hline Cortisol & $\begin{array}{c}3 / 22 \\
(13.6 \%)\end{array}$ & $3.7-5.32$ & $\begin{array}{c}3 / 22 \\
(13.6 \%)\end{array}$ & $20.7-24.4$ & $\begin{array}{c}233 / 2010 \\
(11.6 \%)\end{array}$ & $0.652-6.13$ & 0.7353 & $\begin{array}{c}111 / 2010 \\
(5.5 \%)\end{array}$ & $12.2-35.2$ & 0.122 \\
\hline $\begin{array}{c}\text { Parathyroid } \\
\text { Hormone }\end{array}$ & $\begin{array}{c}1 / 21 \\
(4.8 \%)\end{array}$ & $10.2-10.2$ & $\begin{array}{c}1 / 21 \\
(4.8 \%)\end{array}$ & $\begin{array}{c}78.32- \\
78.32\end{array}$ & $\begin{array}{c}14 / 1709 \\
(0.8 \%)\end{array}$ & $9-14$ & 0.168 & $\begin{array}{c}84 / 1709 \\
(4.9 \%)\end{array}$ & $\begin{array}{c}65.64- \\
465.4\end{array}$ & 1 \\
\hline Estradiol & $\begin{array}{l}17 / 25 \\
(68 \%)\end{array}$ & $5.9-23.92$ & $\begin{array}{c}3 / 25 \\
(12 \%)\end{array}$ & $\begin{array}{c}61.58- \\
113.9\end{array}$ & $\begin{array}{c}1365 / 2071 \\
(65.9 \%)\end{array}$ & $5.02-25.74$ & 0.9945 & $\begin{array}{c}60 / 2071 \\
(2.9 \%)\end{array}$ & $\begin{array}{c}61.22- \\
247.4\end{array}$ & 0.0372 \\
\hline FSH & $\begin{array}{c}5 / 22 \\
(22.7 \%)\end{array}$ & $0.129-1$ & $\begin{array}{c}1 / 22 \\
(4.5 \%)\end{array}$ & $52.2-52.2$ & $\begin{array}{c}323 / 1913 \\
(16.9 \%)\end{array}$ & $0.1-1.44$ & 0.6596 & $\begin{array}{c}43 / 1913 \\
(2.2 \%)\end{array}$ & $12.5-63.9$ & 0.3988 \\
\hline LH & $\begin{array}{c}6 / 23 \\
(26.1 \%)\end{array}$ & $0.3-1.38$ & $\begin{array}{c}3 / 23 \\
(13 \%)\end{array}$ & $8.7-27.1$ & $\begin{array}{c}341 / 1936 \\
(17.6 \%)\end{array}$ & $0.1-1.64$ & 0.4334 & $\begin{array}{c}112 / 1936 \\
(5.8 \%)\end{array}$ & $8.68-53.67$ & 0.1488 \\
\hline Progesterone & $\begin{array}{c}8 / 22 \\
(36.4 \%)\end{array}$ & $\begin{array}{c}0.065- \\
0.182\end{array}$ & $\begin{array}{c}1 / 22 \\
(4.5 \%)\end{array}$ & $\begin{array}{c}0.212- \\
0.212\end{array}$ & $\begin{array}{c}494 / 1801 \\
(27.4 \%)\end{array}$ & $\begin{array}{c}0.052- \\
0.194\end{array}$ & 0.4887 & $\begin{array}{c}66 / 1801 \\
(3.7 \%)\end{array}$ & $\begin{array}{c}0.152- \\
17.02\end{array}$ & 0.5634 \\
\hline $\begin{array}{c}\text { Total } \\
\text { Testosterone }\end{array}$ & $\begin{array}{c}4 / 28 \\
(14.3 \%)\end{array}$ & $22.9-204.1$ & $\begin{array}{c}4 / 28 \\
(14.3 \%)\end{array}$ & $1013-1400$ & $\begin{array}{c}363 / 2286 \\
(15.9 \%)\end{array}$ & $5.5-347.3$ & 1 & $\begin{array}{c}239 / 2286 \\
(10.5 \%)\end{array}$ & $839.6-1499$ & 0.5282 \\
\hline SHBG & $\begin{array}{c}2 / 28 \\
(7.1 \%)\end{array}$ & $8.5-13.7$ & $\begin{array}{c}13 / 28 \\
(46.4 \%)\end{array}$ & $56.6-189.5$ & $\begin{array}{c}138 / 2217 \\
(6.2 \%)\end{array}$ & $3.9-16.44$ & 0.6926 & $\begin{array}{c}364 / 2217 \\
(16.4 \%)\end{array}$ & $\begin{array}{c}56.05- \\
181.7\end{array}$ & 0.0001 \\
\hline DHEA-S & $\begin{array}{c}4 / 25 \\
(16 \%)\end{array}$ & $24.9-153.7$ & $\begin{array}{c}4 / 25 \\
(16 \%)\end{array}$ & $\begin{array}{c}364.2- \\
486.2\end{array}$ & $\begin{array}{c}64 / 2084 \\
(3.1 \%)\end{array}$ & $8-159.3$ & 0.0075 & $\begin{array}{c}176 / 2084 \\
(8.4 \%)\end{array}$ & $331.6-972$ & 0.159 \\
\hline IGF-I & $\begin{array}{l}0 / 12 \\
(0 \%)\end{array}$ & N/A & $0 / 12(0 \%)$ & N/A & $\begin{array}{c}37 / 1042 \\
(3.6 \%)\end{array}$ & $\begin{array}{c}11.99- \\
99.36\end{array}$ & 1 & $\begin{array}{c}42 / 1042 \\
(4 \%)\end{array}$ & $200-474.84$ & 1 \\
\hline Estriol & $0 / 3(0 \%)$ & N/A & $0 / 3(0 \%)$ & N/A & $0 / 181(0 \%)$ & N/A & 1 & $4 / 181(2.2 \%)$ & $\begin{array}{l}208.4- \\
420.49\end{array}$ & 1 \\
\hline Estrone & $\begin{array}{c}1 / 3 \\
(33.3 \%)\end{array}$ & N/A & $0 / 3(0 \%)$ & N/A & $\begin{array}{c}39 / 308 \\
(12.7 \%)\end{array}$ & $9.1-10.1$ & 0.3393 & $\begin{array}{l}17 / 308 \\
(5.5 \%)\end{array}$ & $51.2-88.6$ & 1 \\
\hline Prolactin & $0 / 1(0 \%)$ & N/A & $\begin{array}{c}1 / 1 \\
(100 \%)\end{array}$ & $\begin{array}{c}21.01- \\
21.01\end{array}$ & $3 / 97$ (3.1\%) & $1.91-4.01$ & 1 & $\begin{array}{c}10 / 97 \\
(10.3 \%)\end{array}$ & $\begin{array}{c}15.69- \\
35.85\end{array}$ & 0.1122 \\
\hline
\end{tabular}


Table S31. Age group 36 - 49. Reproductive hormonal changes in men with positive anti-TPO. The significance was not considered when the total number of subjects tested for each hormonal group is $\leq 10$.

\begin{tabular}{|c|c|c|c|c|c|c|c|c|c|c|}
\hline & \multicolumn{4}{|c|}{ Anti-TPO+ $(\mathrm{n}=1949)$} & \multicolumn{6}{|c|}{ Anti-TPO- $(n=610)$} \\
\hline & Reduced & $\begin{array}{c}\text { Range } \\
\text { (reduced) }\end{array}$ & Elevated & $\begin{array}{c}\text { Range } \\
\text { (elevated) }\end{array}$ & Reduced & $\begin{array}{c}\text { Range } \\
\text { (reduced) }\end{array}$ & $\begin{array}{l}\text { P value } \\
\text { (reduced) }\end{array}$ & Elevated & $\begin{array}{c}\text { Range } \\
\text { (elevated) }\end{array}$ & $\begin{array}{l}\text { P value } \\
\text { (elevated) }\end{array}$ \\
\hline Cortisol & $\begin{array}{c}206 / 1676 \\
(12.3 \%)\end{array}$ & $0.652-6.13$ & $\begin{array}{c}74 / 1676 \\
(4.4 \%)\end{array}$ & $\begin{array}{c}12.21- \\
29.27\end{array}$ & $\begin{array}{l}47 / 518 \\
(9.1 \%)\end{array}$ & $0.7-6.12$ & 0.0542 & $\begin{array}{l}50 / 518 \\
(9.7 \%)\end{array}$ & $12.2-35.2$ & 0 \\
\hline $\begin{array}{c}\text { Parathyroid } \\
\text { Hormone }\end{array}$ & $\begin{array}{c}11 / 1412 \\
(0.8 \%)\end{array}$ & 43357 & $\begin{array}{c}64 / 1412 \\
(4.5 \%)\end{array}$ & $\begin{array}{c}65.64- \\
465.4\end{array}$ & $\begin{array}{l}6 / 459 \\
(1.3 \%)\end{array}$ & $10.2-13.3$ & 0.4516 & $\begin{array}{l}25 / 459 \\
(5.4 \%)\end{array}$ & $\begin{array}{c}65.72- \\
158.4\end{array}$ & 0.5009 \\
\hline Estradiol & $\begin{array}{c}1114 / 1731 \\
(64.4 \%)\end{array}$ & $5.02-25.74$ & $\begin{array}{c}59 / 1731 \\
(3.4 \%)\end{array}$ & $\begin{array}{c}61.22- \\
247.4\end{array}$ & $\begin{array}{l}380 / 540 \\
(70.4 \%)\end{array}$ & $5.2-25.7$ & 0.0117 & $\begin{array}{l}17 / 540 \\
(3.1 \%)\end{array}$ & $\begin{array}{c}61.57- \\
316.7\end{array}$ & 0.8756 \\
\hline FSH & $\begin{array}{c}255 / 1579 \\
(16.1 \%)\end{array}$ & $0.1-1.44$ & $\begin{array}{c}38 / 1579 \\
(2.4 \%)\end{array}$ & $12.5-63.9$ & $\begin{array}{l}103 / 504 \\
(20.4 \%)\end{array}$ & $0.1-1.3$ & 0.0313 & $\begin{array}{c}10 / 504 \\
(2 \%)\end{array}$ & $12.5-25.45$ & 0.7041 \\
\hline LH & $\begin{array}{c}277 / 1591 \\
(17.4 \%)\end{array}$ & $0.1-1.64$ & $\begin{array}{c}93 / 1591 \\
(5.8 \%)\end{array}$ & $8.68-53.67$ & $\begin{array}{l}107 / 516 \\
(20.7 \%)\end{array}$ & $0.1-1.56$ & 0.102 & $\begin{array}{l}30 / 516 \\
(5.8 \%)\end{array}$ & $8.7-17$ & 1 \\
\hline Progesterone & $\begin{array}{c}436 / 1489 \\
(29.3 \%)\end{array}$ & $\begin{array}{c}0.052- \\
0.194\end{array}$ & $\begin{array}{c}63 / 1489 \\
(4.2 \%)\end{array}$ & $0.152-6.29$ & $\begin{array}{l}107 / 474 \\
(22.6 \%)\end{array}$ & $0.053-0.19$ & 0.0054 & $\begin{array}{l}13 / 474 \\
(2.7 \%)\end{array}$ & $\begin{array}{c}0.175- \\
17.02\end{array}$ & 0.1848 \\
\hline $\begin{array}{c}\text { Total } \\
\text { Testosterone }\end{array}$ & $\begin{array}{c}275 / 1912 \\
(14.4 \%)\end{array}$ & $\begin{array}{c}10.95- \\
347.3\end{array}$ & $\begin{array}{c}205 / 1912 \\
(10.7 \%)\end{array}$ & $\begin{array}{c}839.6- \\
1499\end{array}$ & $\begin{array}{l}128 / 595 \\
(21.5 \%)\end{array}$ & $5.5-342.9$ & 0 & $\begin{array}{c}61 / 595 \\
(10.3 \%)\end{array}$ & $\begin{array}{c}841.7- \\
1491\end{array}$ & 0.8036 \\
\hline SHBG & $\begin{array}{c}105 / 1860 \\
(5.6 \%)\end{array}$ & $4.23-16.44$ & $\begin{array}{c}323 / 1860 \\
(17.4 \%)\end{array}$ & $\begin{array}{c}56.05- \\
189.5\end{array}$ & $\begin{array}{l}49 / 581 \\
(8.4 \%)\end{array}$ & $3.9-16.4$ & 0.0206 & $\begin{array}{c}76 / 581 \\
(13.1 \%)\end{array}$ & $56.2-187.2$ & 0.0176 \\
\hline DHEA-S & $\begin{array}{c}59 / 1738 \\
(3.4 \%)\end{array}$ & $8-159.3$ & $\begin{array}{c}149 / 1738 \\
(8.6 \%)\end{array}$ & $\begin{array}{c}331.6- \\
884.6\end{array}$ & $\begin{array}{l}15 / 549 \\
(2.7 \%)\end{array}$ & $24.9-153.7$ & 0.5311 & $\begin{array}{c}44 / 549 \\
(8 \%)\end{array}$ & $338.6-972$ & 0.7472 \\
\hline IGF-I & $25 / 830(3 \%)$ & $15.4-94.47$ & $\begin{array}{l}31 / 830 \\
(3.7 \%)\end{array}$ & $\begin{array}{c}200- \\
386.53\end{array}$ & $\begin{array}{c}18 / 302 \\
(6 \%)\end{array}$ & $\begin{array}{c}11.99 \text { - } \\
99.36\end{array}$ & 0.0341 & $\begin{array}{l}14 / 302 \\
(4.6 \%)\end{array}$ & $\begin{array}{c}203- \\
474.84\end{array}$ & 0.6072 \\
\hline Estriol & $0 / 185(0 \%)$ & N/A & $\begin{array}{l}4 / 185 \\
(2.2 \%)\end{array}$ & $\begin{array}{l}208.4- \\
420.49\end{array}$ & $0 / 19(0 \%)$ & N/A & 1 & $0 / 19(0 \%)$ & N/A & 1 \\
\hline Estrone & $\begin{array}{c}39 / 312 \\
(12.5 \%)\end{array}$ & $9.5-10.1$ & $\begin{array}{l}18 / 312 \\
(5.8 \%)\end{array}$ & $51.3-88.6$ & $\begin{array}{c}6 / 36 \\
(16.7 \%)\end{array}$ & $9.1-9.1$ & 0.6576 & $\begin{array}{c}3 / 36 \\
(8.3 \%)\end{array}$ & $51.2-60.46$ & 0.4667 \\
\hline Prolactin & $2 / 96(2.1 \%)$ & $1.91-4.01$ & $\begin{array}{c}13 / 96 \\
(13.5 \%)\end{array}$ & $\begin{array}{c}15.69- \\
35.85\end{array}$ & $1 / 14(7.1 \%)$ & $3.6-3.6$ & 0.338 & $\begin{array}{c}2 / 14 \\
(14.3 \%)\end{array}$ & $\begin{array}{c}19.15- \\
24.09\end{array}$ & 1 \\
\hline
\end{tabular}

Table S32. Age group 36 - 49. Reproductive hormonal changes in men with positive anti-Tg. The significance was not considered when the total number of subjects tested for each hormonal group is $\leq 10$.

\begin{tabular}{|c|c|c|c|c|c|c|c|c|c|c|}
\hline & \multicolumn{4}{|c|}{ Anti-Tg+ $(\mathrm{n}=1499)$} & \multicolumn{6}{|c|}{ Anti-Tg- $(n=1061)$} \\
\hline & Reduced & $\begin{array}{c}\text { Range } \\
\text { (reduced) }\end{array}$ & Elevated & $\begin{array}{c}\text { Range } \\
\text { (elevated) }\end{array}$ & Reduced & $\begin{array}{c}\text { Range } \\
\text { (reduced) }\end{array}$ & $\begin{array}{l}\text { P value } \\
\text { (reduced) }\end{array}$ & Elevated & $\begin{array}{c}\text { Range } \\
\text { (elevated) }\end{array}$ & $\begin{array}{c}\text { P value } \\
\text { (elevated) }\end{array}$ \\
\hline Cortisol & $\begin{array}{c}148 / 1277 \\
(11.6 \%)\end{array}$ & $0.7-6.11$ & $\begin{array}{c}71 / 1277 \\
(5.6 \%)\end{array}$ & $12.2-35$ & $\begin{array}{l}105 / 916 \\
(11.5 \%)\end{array}$ & $0.652-6.13$ & 0.9809 & $\begin{array}{l}53 / 916 \\
(5.8 \%)\end{array}$ & $13.07-35.2$ & 0.8947 \\
\hline $\begin{array}{c}\text { Parathyroid } \\
\text { Hormone }\end{array}$ & $\begin{array}{c}10 / 1087 \\
(0.9 \%)\end{array}$ & $10.9-14$ & $\begin{array}{c}50 / 1087 \\
(4.6 \%)\end{array}$ & $\begin{array}{c}65.64- \\
465.4\end{array}$ & $\begin{array}{l}7 / 783 \\
(0.9 \%)\end{array}$ & $9-13.84$ & 1 & $\begin{array}{c}39 / 783 \\
(5 \%)\end{array}$ & $\begin{array}{c}65.72- \\
138.4\end{array}$ & 0.7858 \\
\hline Estradiol & $\begin{array}{c}881 / 1333 \\
(66.1 \%)\end{array}$ & $5.02-25.74$ & $\begin{array}{c}38 / 1333 \\
(2.9 \%)\end{array}$ & $\begin{array}{c}61.22- \\
316.7\end{array}$ & $\begin{array}{l}613 / 938 \\
(65.4 \%)\end{array}$ & $5.3-25.7$ & 0.7483 & $\begin{array}{l}38 / 938 \\
(4.1 \%)\end{array}$ & $\begin{array}{c}61.58- \\
171.8\end{array}$ & 0.1477 \\
\hline FSH & $\begin{array}{c}223 / 1222 \\
(18.2 \%)\end{array}$ & $0.1-1.37$ & $\begin{array}{c}27 / 1222 \\
(2.2 \%)\end{array}$ & $12.5-52.2$ & $\begin{array}{l}135 / 863 \\
(15.6 \%)\end{array}$ & $0.1-1.44$ & 0.1349 & $\begin{array}{l}21 / 863 \\
(2.4 \%)\end{array}$ & $12.86-63.9$ & 0.8513 \\
\hline
\end{tabular}




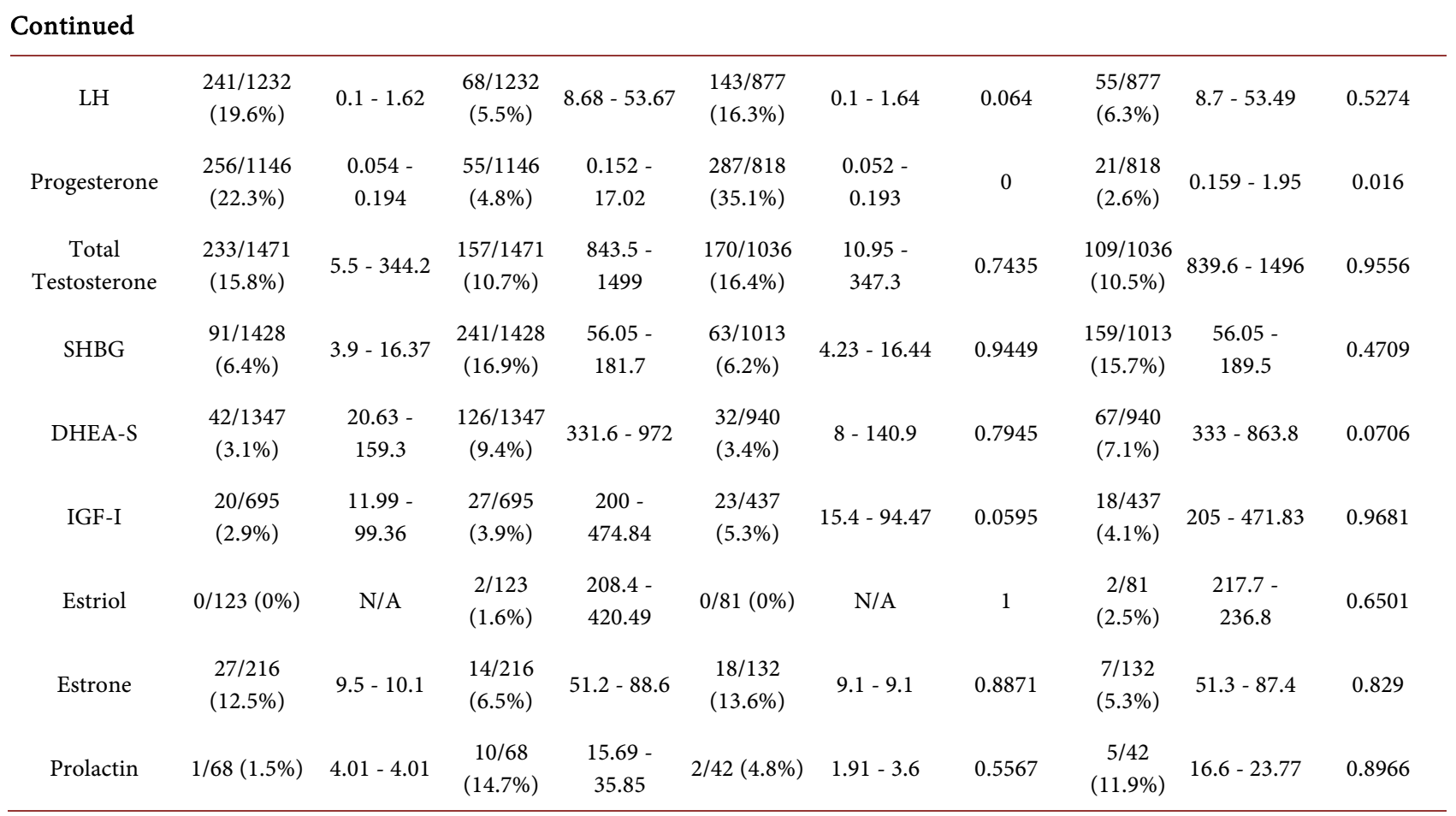

